\title{
DOI: https://doi.org/10.24297/jap.v19i.9115
}

\section{Common-Sense Rejected by Physicists:}

\author{
A Level-Headed Approach to Time and Quantum Weirdness \\ Author: Jeffrey H. Boyd* \\ Affiliation: * Retired. 57 Woods Road, Bethany, CT, 06524-3103 USA \\ Jeffrey.Boyd@alumni.harvard.edu
}

\begin{abstract}
:
We propose a new integration of relativity and quantum mechanics (QM). Your cell phone or smart phone is a rich source of empirical information about relativity. It tells time based on a system called Coordinated Universal Time (UTC) which assumes absolute simultaneity: all observers in all inertial frames observe the same sequence of all events. You must choose whether to trust the time on your cell phone, or trust Einstein's incompatible ideas about a space-time continuum. As concerns QM, the existence of "weirdness" means a mistake was made in QM's starting assumptions. This article finds and corrects that mistake and presents for the first time, a quantum world free of all weirdness. There is another half to nature, previously unrecognized. It is devoid of energy and matter, namely zero-energy Elementary Waves which move within the medium of aether. We derive the linear wave PDE's. There is evidence that Elementary Waves are in control of nature, despite their lack of energy. The existence of UFO's (Unidentified Flying Objects) suggests that someone has learned how to control Elementary Waves. If we could learn from the UFO's, we might acquire a decisive advantage in our battle against climate change.
\end{abstract}

Academic Discipline and Sub-Disciplines: Alternative Quantum Physics, simultaneity, International Atomic Time, Special Relativity, space-time continuum, Unidentified Flying Objects, climate change

Type (Method/Approach): There are three presentations of the same material. This article is a scholarly presentation in a peer reviewed journal. It is parallel to two YouTube videos which are livelier, more entertaining but lack mathematical equations, covering much (but not all) of the same material.

\section{Introduction}

This article presents a non-Einstein view of time, namely the Coordinated Universal Time (UTC) that is used by your cell phone. We also attack the problem of quantum weirdness from an angle never before used, and we identify and eliminate quantum weirdness by postulating another half to nature, one devoid of matter and energy, one grounded in the UTC. (4-31)

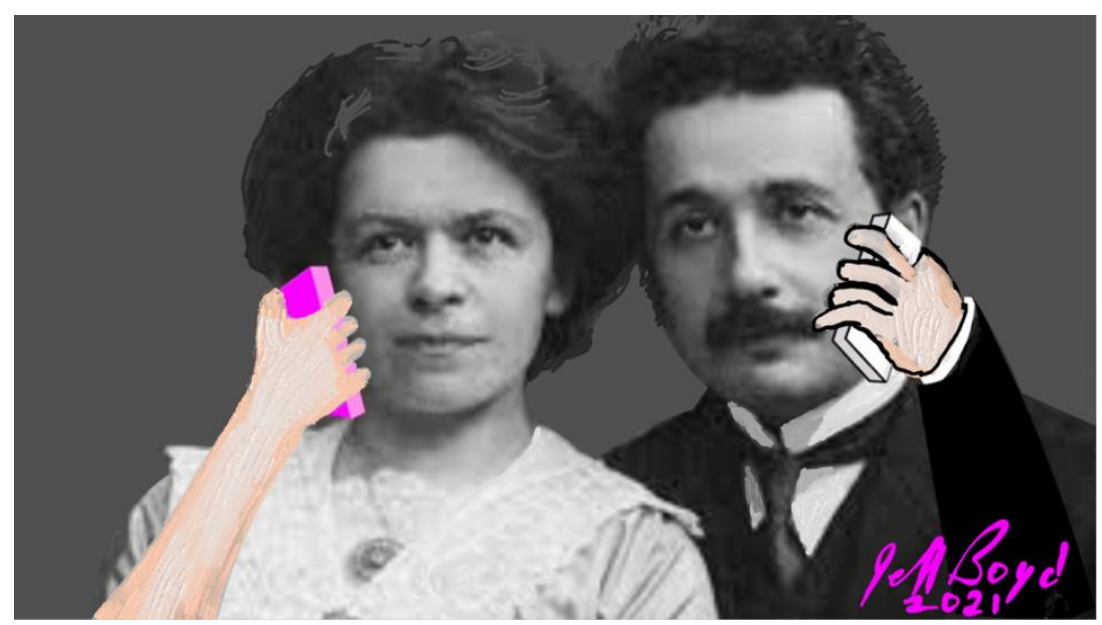

Fig. 1. Mileva and Albert Einstein in 1905 with the smart phones they never had. 
We have presented these common-sense ideas more than a dozen times to the American Physical Society. Audiences respond with blank faces, no comments and no questions. It appears these ideas are so unorthodox and eccentric that scholars are speechless. One quantum expert told us, "I don't want to waste my time thinking about quantum weirdness." We interpret the scholarly disinterest in our ideas as an honour. Many great discoveries of science in the past have rendered mainstream scientists mute. In his study of scientific revolutions, Thomas Kuhn found that paradigm shifts usually sound like unintelligible gibberish to the leaders trained in the previous paradigm. (38)

The first half of this article is about relativity, the second half quantum mechanics. Both halves are about common-sense. They also share aether.

Most of the content of this article can be found in two lively and entertaining YouTube videos with no mathematical equations. One is seven minutes long, the other one hour. Those videos are an easier way to first learn this material than is this article with its mathematical equations. Each video is named "Common-sense rejected by physicists". They are on the YouTube channel named "Elwavetheory" (YouTube will redirect you to "Elwave Theory" in which case you need to insist on "Elwavetheory"). If you can't find the videos, you may have to wait until they are posted, which will be before the end of 2021, probably by October 2021. If you continue to have trouble finding the videos, check the website ElementaryWave.com.

\section{A non-Einstein approach to Relativity}

Einstein never had a smart phone. He would have thought very differently about time if he had. Imagine a conversation between Einstein at the Swiss Patent Office with his smart phone and his wife Mileva with her iPhone at the Train Station in Bern. $(35,56-63)$

Albert: "So when does the train leave for Zürich?"

Mileva: "In 25 minutes, at 3:32."

Albert: "OK. And what time is it now where you are?"

Mileva: "3:07."

Albert: "That is very odd. It is 3:07 over here at the Patent Office also."

Mileva: "Of course."

Albert: "Don't say 'Of course.' It is unusual that the time where I am is exactly the same as where you are. How unlikely is that! When I snap my fingers, tell me the exact number of seconds, so we can see the precise difference in the time where you are and where I am. OK, SNAP!"

Mileva: "It was 3:08 and nine seconds."

Albert: "That is completely weird. That is precisely the same time where I am! What an unusual coincidence!"

Mileva: "Sweetheart, I've been trying to tell you that your ideas about no simultaneity are wrong. But you won't listen to me. Can your smart phone teach you what you won't hear from your wife?"

Albert: "The rule is that two observers can never agree about whether two events are simultaneous or not. I cannot comprehend why the time on your cell phone just happens, by accident, to be simultaneous with the time on my cell phone."

What we mean by the word "time" has changed profoundly. In 1905 every clock ticked at its own rate, and the time on different clocks would differ by several seconds. Time was local. There was no practical way to standardize time. Here is how Einstein defines what "7 o'clock" meant in 1905:

"If I say, 'That train arrives here at 7 o'clock,' I mean something like this: 'The pointing of the small hand of my watch to 7 and the arrival of the train are simultaneous events.' " 
In other term, the time "7 o'clock" was an approximate phenomenon localized to the face of his watch or clock.

That is not how we define, nor how we experience time in the twenty-first century! What we mean by term "7 o'clock" today is that all cell phones around the planet totally agree with each other down to the nanosecond and there is a network in which all phones link to cell towers in a UTC system of absolute simultaneity. "Absolute" means that all observers in all inertial frames agree on the sequence of all events. Whereas "7 o'clock" was a local phenomenon in 1905, today it is the state of a vast network of computers and cell towers. World time is displayed in 24 different time zones, depending on longitude. But even in distant time zones the number of minutes, seconds, milliseconds, microseconds, nanoseconds, picosecond and femtoseconds is always the same.

The two views are incompatible. Einstein's ideas about lack of simultaneity and the existence of a spacetime continuum are incompatible with the idea of absolute simultaneity used by the UTC. Today you are confronted, as we said, by the need to trust the time on your cell phone and mistrust Einstein's ideas about time, or vice versa. Relativity experts are not aware of this dilemma.

\section{1. "Absolute Simultaneity" defined using Georg Cantor's set theory}

Absolute simultaneity does not mean "absolute" in all respects. It means there is no spatial aspect to time. There is no space-time continuum. But there is still time dilation if a clock is subjected to more gravity or more velocity. This can be clarified by Georg Cantor's vocabulary.

The words "simultaneous" and "synchronized" are related. "Simultaneous" means that two events, no matter how far apart, that are viewed by one observer as occurring at the same time, are viewed by all observers as occurring at the same time. This is a fundamental property of the universe. The word "synchronized" means that humans can set two clocks so they tell the same time, and continue to tell the same time forever, when supervised by a computer that corrects for the local clock ticking too fast or too slow. Georg Cantor's set theory provides the vocabulary we need to understand this.

We will define the time on clocks $\boldsymbol{\alpha}$ and $\boldsymbol{\beta}$ as being ordered sets of real numbers that are "similar". An ordered set $\mathrm{M}$ is defined by Cantor as "similar to ordered set $\mathrm{N}$ ", when the elements of $\mathrm{M}$ and $\mathrm{N}$ can be put into a one-to-one correspondence in such a manner that when any two elements of $M_{1} m_{1}$ and $m_{2}$ the relation $m_{1} \prec m_{2}$ holds, then for the corresponding elements $n_{1}$ and $n_{2}$ of $N$, the relation $n_{1} \prec n_{2}$ also holds. In this case we write "N $\simeq M^{\prime}$ and say "N is similar to $M$."

The idea of "absolute simultaneity" used in this article means that an ordered set of all events in one inertial frame is always "similar to" an ordered set of all events in a different inertial frame. Einstein had said that was impossible. Thus if one inertial frame $M$ is where you are located, while the other inertial frame $N$ is closer to the center of the earth, where there is time dilation, then we can write " $N \simeq M$ " and say "N is similar to M." That which is "absolute" is the ordering of each sequence of events. The word "absolute" does not mean that whenever a clock ticks one second in frame $M$, the other clock in frame $\mathrm{N}$ ticks one second.

\subsection{Coordinated Universal Time (UTC)}

Einstein said two observers can never agree on the sequence of simultaneous events. If Einstein were correct about simultaneity, then all stock markets on earth would crash in less than one second.

Most trading on stock exchanges is not done by humans but by computers. If one computer was to say (based on nanoseconds), that the sequence of events was ABCDEF, but the other computer was to say, no, no the sequence was ACBDFE, then the whole network would crash in less than a second. If one computer was to say, "I bought that stock before I sold it," but the other computer was to say, "Well, you can march to your own drummer, but you sold that stock before you bought it!", then the financial markets would collapse, and world trade would stop. Since atomic clocks tell time in femtoseconds, most computers are in different inertial frames of reference, if you consider the rotation of the earth. 
Since 1980 everyone on earth, including you, have been living under the umbrella of an international agreement written in French: the "Advisory Committee for the Definition of the Second" (CCDS = "Comité Consultatif pour la Définition de la Seconde") by the International Bureau of Weights and Measures (BIPM = "Bureau International des Poids et Mesures") in Saint-Cloud, France. This is the bureau charged with defining the International System of Units (SI) used throughout science: kilograms, meters, seconds. It defines International Atomic Time (IAT), according to which one second is the time it takes a Cesium-133 atom at the ground state to oscillate 9,192,631,770 times. The CCDS tells how to synchronize atomic clocks if they are moving on what Einstein would call "different spaceships." Since the speed of the earth's $2 \pi$ rotation fluctuates slightly (Fig. 2), the official time of the world is the UTC which uses both the IAT and the earth's current speed of rotation. UTC is sometimes called "Zulu military time."(48)

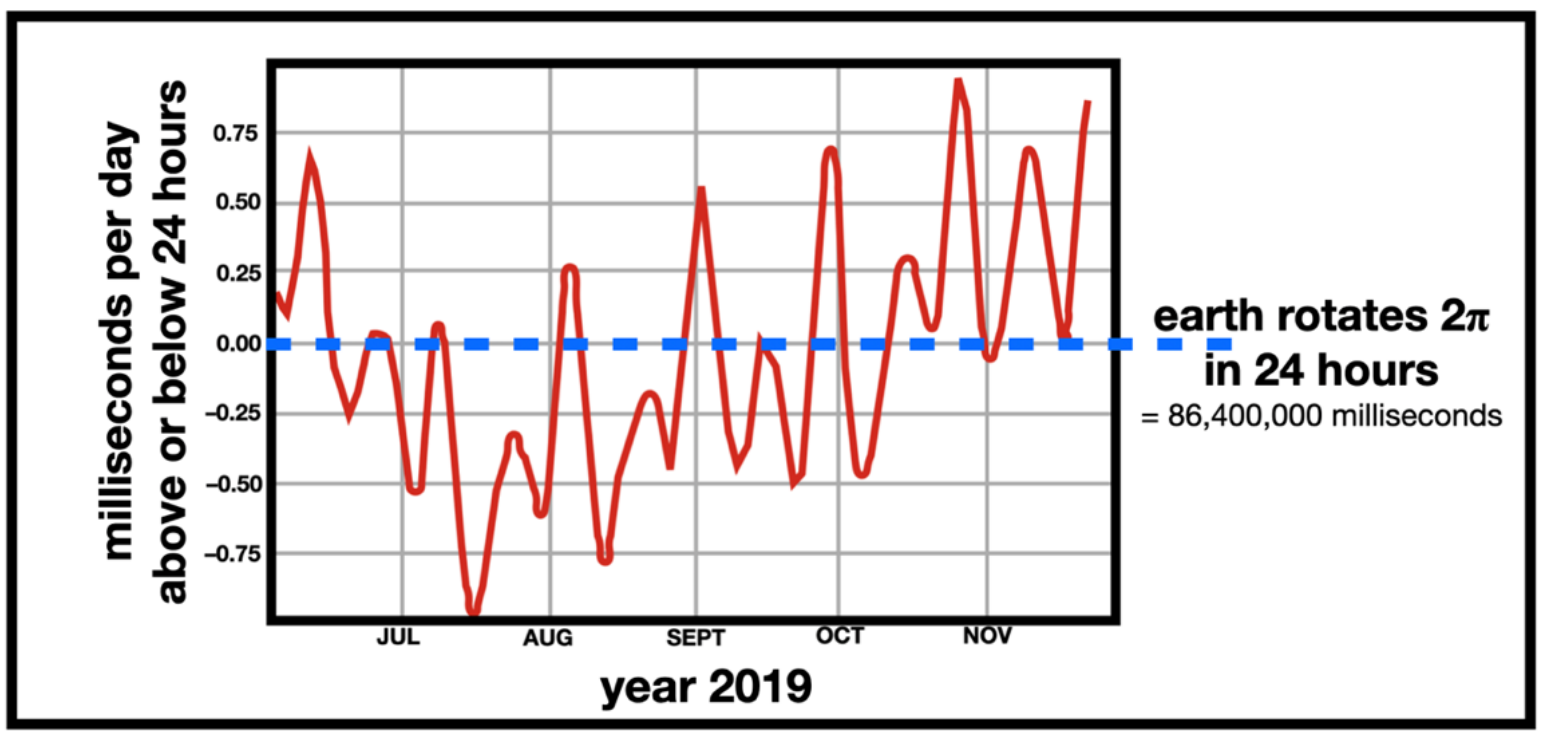

Fig. 2. The time it takes the earth to make one full rotation $(2 \pi)$ can vary from day to day by almost two milliseconds. Given conservation of angular momentum, this implies that heavy rivers of magma are moving upward or downward deep inside the earth. The UTC defines "one second" based on a coordination of International Atomic Time, and the day-to-day speed of the earth's rotation.

The SI definition of a second of time contradicts Einstein, and it is the SI definition of time that you find displayed on your cell phone. It is no longer the case that the term "7 o'clock" means "The pointing of the small hand of my watch to $7 . "$

Einstein also said that the speed of light is the same for all observers. He also described a space-time continuum. You can learn from your cell phone that Einstein was wrong, as we will now show.

\subsection{The CCDS (1980) established absolute simultaneity everywhere on earth}

In 1980 the BIPM established how to set two atomic clocks to the same time, even if they were in different inertial frames. That document (CCDS) defined a "second" in International Atomic Time in the SI system. The method of coordinating time in two atomic clocks (which might be orbiting the earth in different satellites) contradicts how Einstein thought two "spaceships" should coordinate time.

The CCDS defines two ways to synchronize the time so it is identical on any two atomic clocks, and three adjustments to be made no matter which method you use. The two methods are to take one clock physically to where the other clock is located and set them to the same time. The other method is to send a signal from one to the other. 


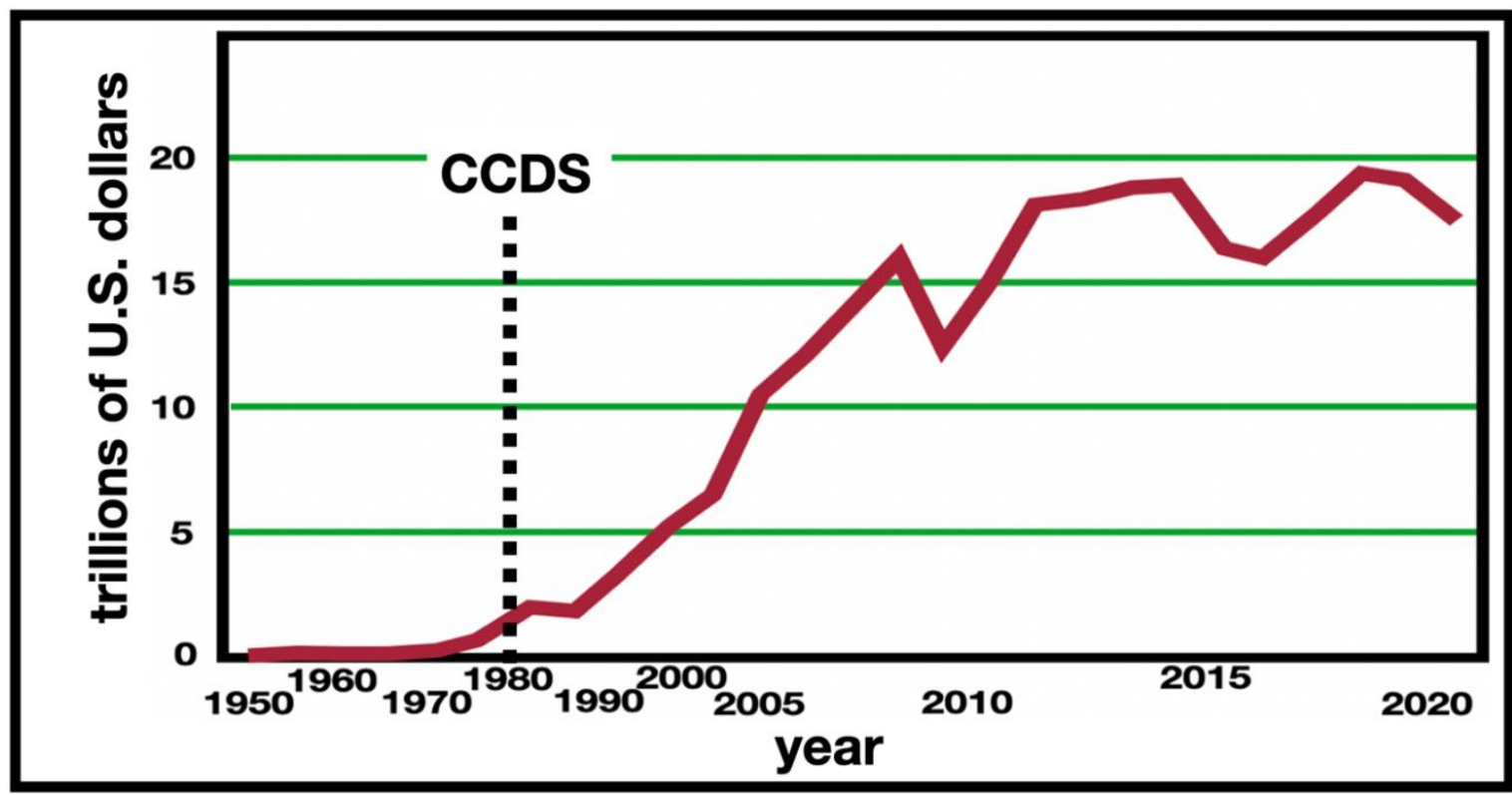

Fig. 3. World trade in trillions of U.S. dollars, made possible by the CCDS agreement in 1980.

No matter which method is used, three adjustments are made by a computer supervising each clock.

First, adjust for gravity because General Relativity correctly predicts slower time (time dilation) with more gravity.

$$
g(\phi) h / c^{2}
$$

where $g$ is the total acceleration (gravitational and centrifugal) at sea level at latitude $\Phi, h$ is the height above sea level and $c$ is the speed of light.

Second, adjust for the speed of the satellite or airplane carrying a clock, because the Lorentz transformation equations correctly predict time dilation with velocity. (44)

$$
v^{2} / 2 c^{2}
$$

where $v$ is the speed of the airplane or satellite. A satellite in an elliptical orbit might carry four atomic clocks that need to be perpetually updated as the speed and altitude (gravity) change.

Third, adjust for the Sagnac effect (Fig. 4). Before we give that Sagnac equation used by the CCDS, we need to explain what the Sagnac effect is, namely a rotating intertial frame of reference that has different effects on a photon travelling clockwise versus counterclockwise. (51-53)

Fig. 4 pictures an interferometer rotating at two full rotations per second. A photon is emitted by a source in that rotating frame of reference. The photon is split by a beam-splitter into two photons that go around the interferometer in opposite directions, and if the interferometer is itself spinning in a clockwise direction, then the photon travelling counterclockwise will get to the detector faster than the photon that travels clockwise, because it had less distance to go. Thus, from the vantage point of the detector in its inertial frame, the speed of light is faster counterclockwise than clockwise. If the direction of spin is reversed, the speed of light is faster clockwise than counterclockwise. This contradicts what Einstein said about the speed of light being the same in all inertial frames. Sagnac says it proves the existence of a stationary frame of reference, which he called "luminiferous aether." [Note: "aether" is sometimes spelled "ether" or "æther."] (51-53) 


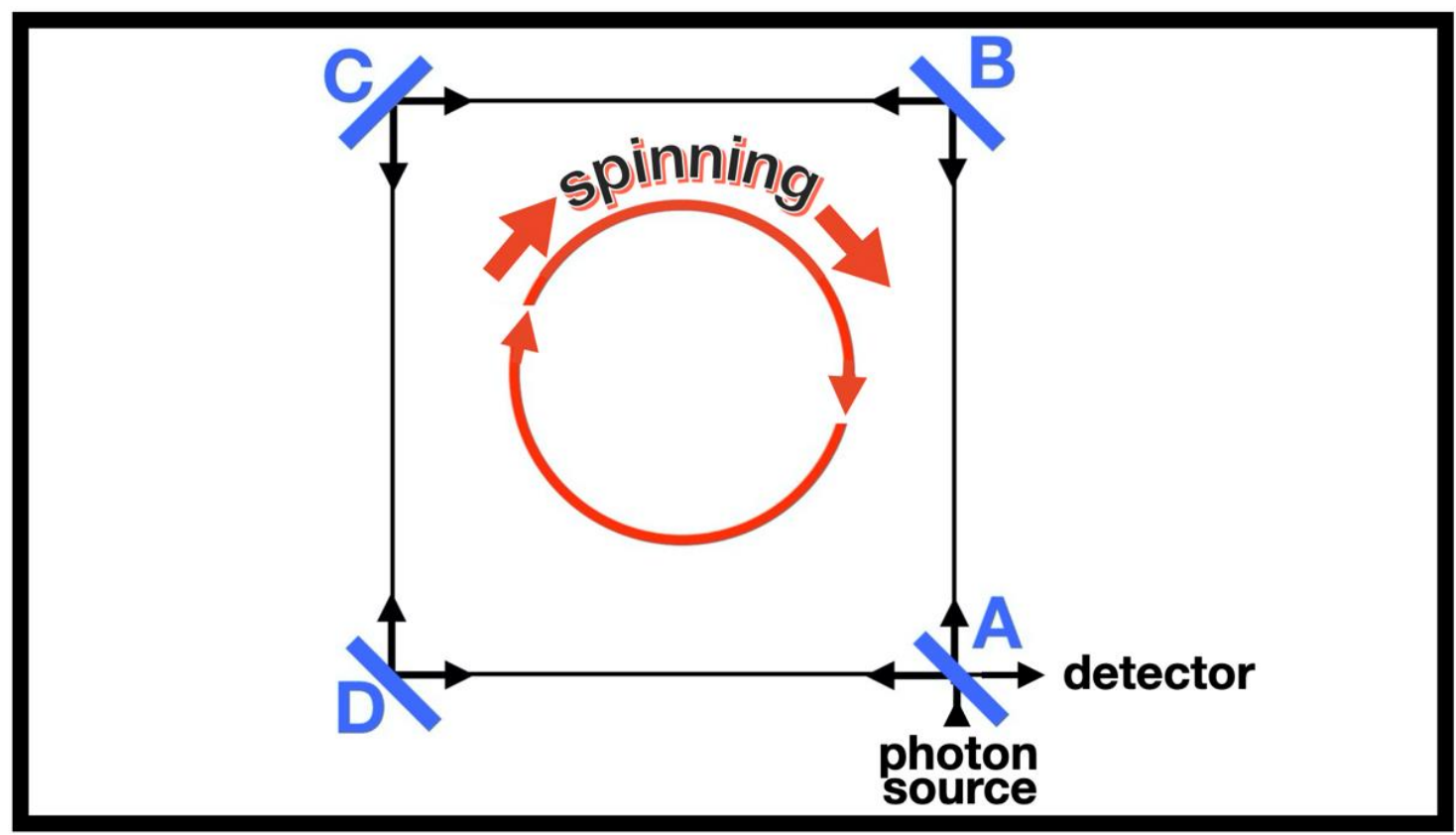

Fig. 4. Sagnac built this interferometer. The entire apparatus spins at least 2 full rotations per second.

The blue lines are mirrors, except for A which is a beam splitter. A photon travelling around the perimeter counterclockwise reaches the detector before a photon travelling around the interferometer in a clockwise direction, because of the rotation of the interferometer, the photon travelling counterclockwise had less distance to go. Since the interferometer is the inertial frame within which the detector is standing, the speed of light is faster counterclockwise than clockwise.

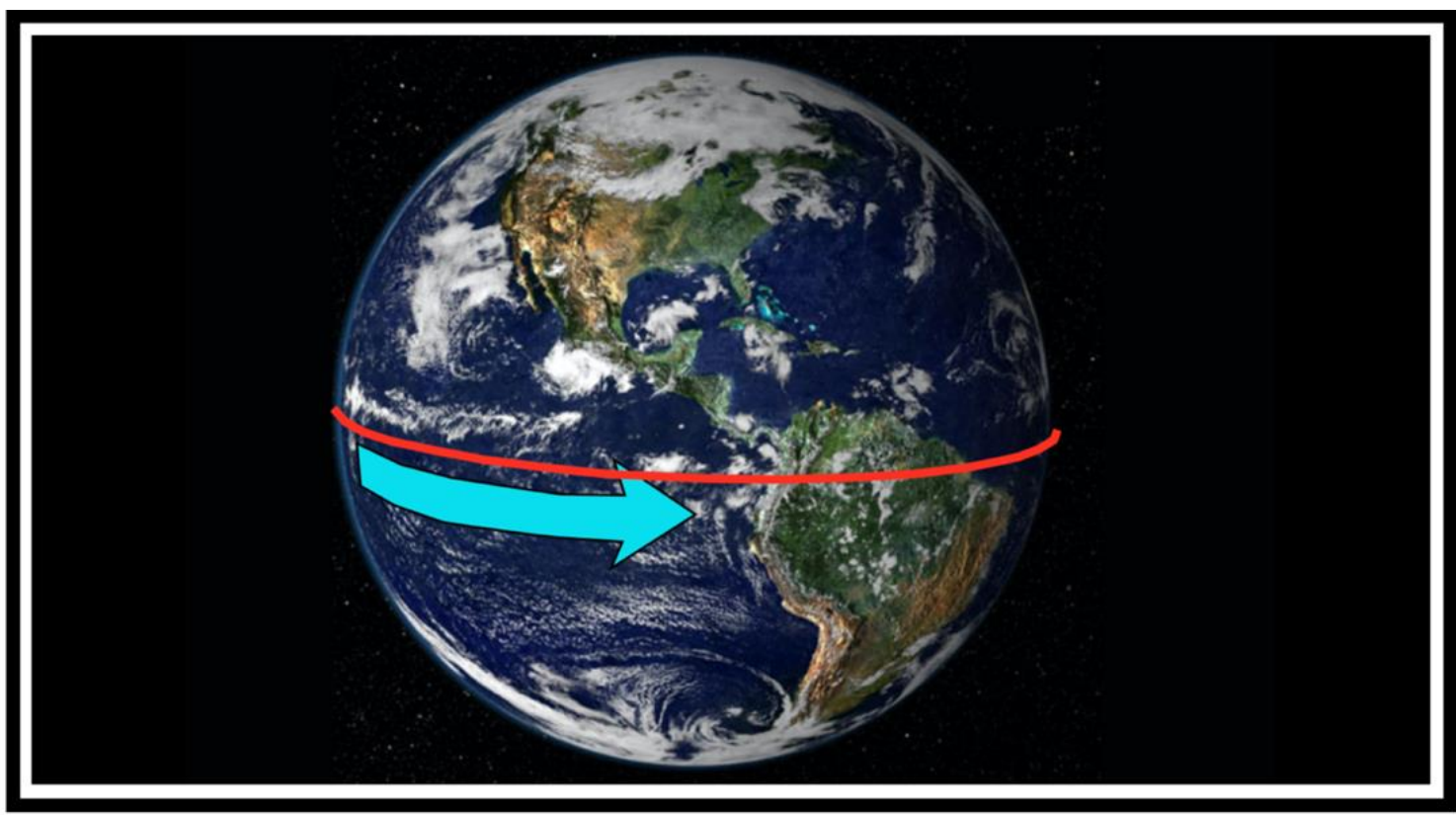

Fig. 5: The earth is a Sagnac interferometer (i.e. a rotating interferometer). If microwave signals were sent both ways around the equator at lightspeed, it would take 414 ns less time travelling westward than travelling eastward, due to the earth's rotation. In the 0.135 seconds it took for the signal to circumnavigate the earth, the earth would rotate 62 meters eastward, shortening the distance westward 
and lengthening the distance eastwards by 62 meters. Conclude: from a human viewpoint the speed of light to the west is faster than the speed of light to the east.

The authors of the CCDS, tiptoeing cautiously around the academic relativity experts, avoided mentioning the name "Georges Sagnac," because they feared that name might be a red flag for relativity experts. Sagnac's three publications (of 1913-1914) were never translated from French, just the CCDS agreement was never translated from French to English. Why the fear? Because Sagnac had proved that luminiferous aether exists and therefore Einstein's Theory of Special Relativity (TSR) was wrong in discarding aether. It was easier to keep relativity experts uninformed, than to provoke an argument. Even today, half a century later, relativity experts are oblivious to the contradiction between Einstein's TSR and the time displayed on their own cell phones.

Sagnac had a major impact on the CCDS, just as he had a major impact on the inertial guidance systems in airplanes and rockets, in the form of ring laser gyroscopes. But because of the fear of awakening relativity experts from their ignorance, CCDS experts didn't mention Sagnac's name.

Fig. 5 shows that in the earth's inertial frame, the speed of light is faster to the west than to the east, contradicting Einstein. However, if you insert a mirror so the signal circumnavigates the earth westward then reflects and circumnavigates it eastward, you will discover that Einstein is correct: the two-way speed of light is always $\mathbf{c}$. Thus the "two-way" speed of light is $\mathbf{c}$, but the one-way speed of light often is not.

In 1905 when Einstein was making his erroneous statements about time and the speed of light, there was no way to test the one-way speed of light. The Michelson-Morley experiment only tested the twoway speed of light. Mathematician Henri Poincaré suggested that since the math was easier if one assumed the one-way speed of light was the same in either direction, therefore it was expedient to assume that to be true. Einstein adopted Poincaré's suggestion, making it sound like a law of nature.

To adjust for the Sagnac effect (clocks moving westward click faster than clocks moving eastward) the CCDS used the following equation, which they described in arcane language for the purpose of keeping you baffled and mystified.

$$
2 A_{E} \omega / c^{2}
$$

Here is what they wrote: "Where $A_{E}$ is the equatorial projection of the area enclosed by the trajectory traveled by the clock (or by the signal) and by the lines connecting the sites of the two clocks with the center of the Earth," and $\omega$ is the angular velocity of the Earth. If you find that sentence confusing, then you should know that it was written for the purpose of derailing your thinking.

The last thing CCDS authors wanted was for physicists to understand Eq. 3. That equation implies that the speed of light travelling westward is faster than the speed of light travelling eastward.

It is easy to see why the CCDS would place so much emphasis on the Sagnac effect, because unless they corrected for it, atomic clocks on satellites, ships, and airplanes travelling to the west would tick faster than atomic clocks travelling to the east.

\subsection{Franco Selleri (1936-2013)}

These ideas about relativity come from a physicist named Franco Selleri. He was at the University of Bari, Italy, and he was an outsider with a colorful career in academic physics. $(35,56-63)$

Early in his physics career Selleri became internationally famous for an idea he had about particle physics. That led to a career in which he spent years at CERN, and at various universities around the United States and Europe as one of the leading young physicists. He discovered that empirical research in subatomic physics was hobbled by the absence of a theoretical foundation for quantum mechanics. Like half of physicists, he found the Copenhagen Interpretation unsatisfactory. (35) 
With encouragement from Louis de Broglie, Karl Popper, and many others, Selleri set about to build a coherent way to understand the quantum world. He loved the optics research by Robert Pfleegor and Leonard Mandel which proved that wave-particle duality is wrong, as we will discuss below in Section 3.6. (14) Selleri never considered the possibility that these zero-energy quantum waves might be travelling in the opposite direction as the particles. As Selleri became more interested in the fundamentals of QM, other physicists lost interest in him.

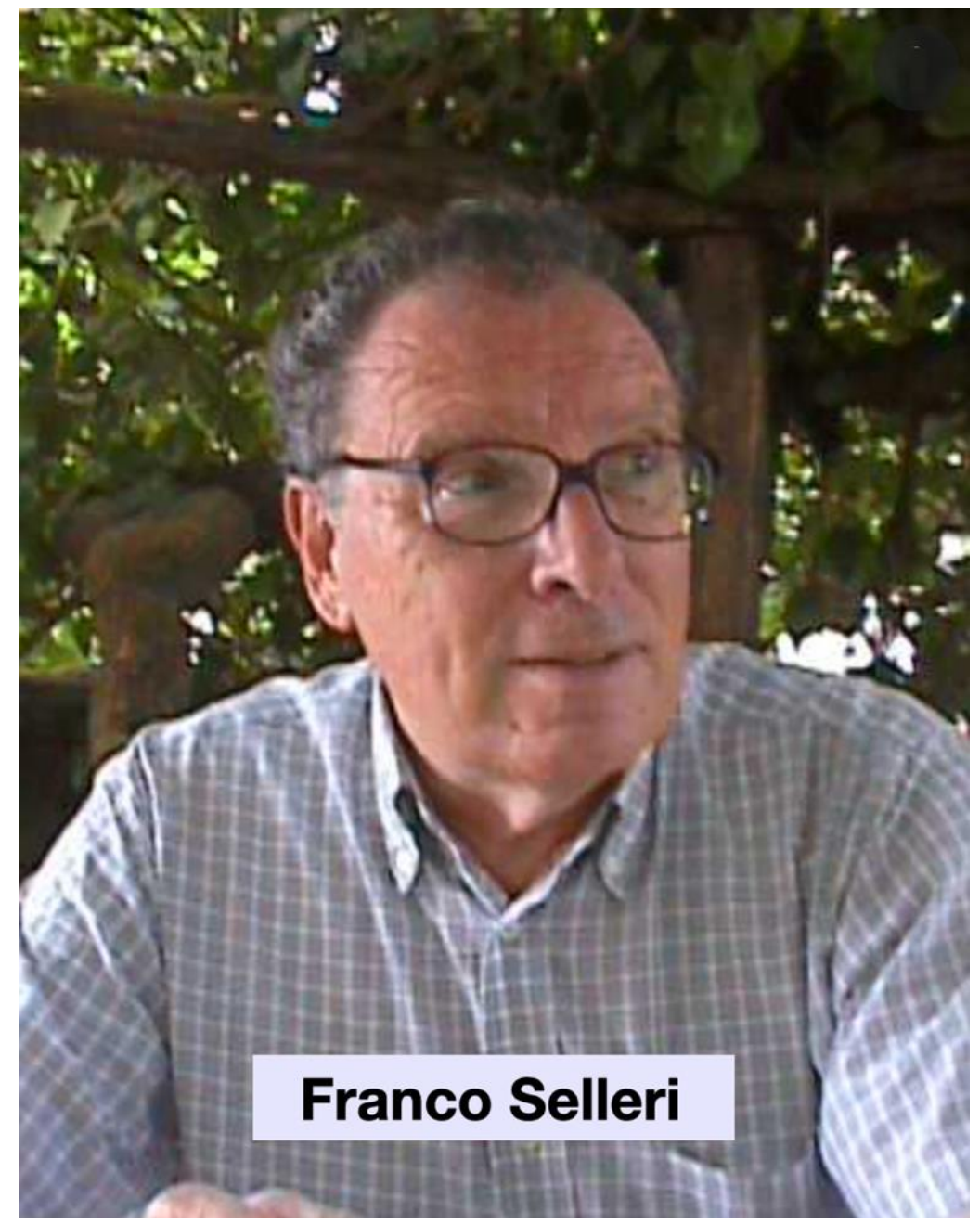

Fig. 6. Franco Selleri (1936-2013) at University of Bari, Italy, established his own relativity theory based on absolute simultaneity. It is the only relativity theory that is built on simultaneity as the central issue.

He became a full professor at the University of Bari, in charge of their nuclear reactor. At international conferences on quantum physics, young physicists would tell Selleri that his ideas about quantum mechanics were fascinating, but it was too dangerous for them to do empirical research along the lines that Selleri suggested. If they did, they said, then either they would prove that the Copenhagen interpretation was wrong, or that it was correct. Either way they would lose. If the Copenhagen interpretation proved to be wrong, then they would be ostracized, and their lab would never get funding again because no one was allowed to contradict the Copenhagen orthodoxy. If the data showed that Copenhagen was correct, then they would be ostracized and never get funding again because they would be said to have wasted money proving what everyone knew to be correct. In either case their reputation would be ruined, and they would have to lay-off their staff, and never become full professors.

After decades of Selleri being ostracized for his criticism of the Copenhagen orthodoxy, one young physicist told Selleri that there needs to be a medium in which Selleri's "quantum waves" travel. That

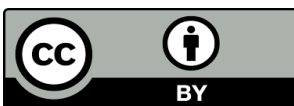


comment led Selleri to switch his interest from quantum mechanics to relativity. For the last decade of his life, he developed his unique theory of relativity based on the concept of absolute simultaneity. His ideas about aether were more popular among physicists than his ideas about zero-energy quantum waves had been. Since Selleri based his relativity on the foundation of absolute simultaneity, he was in complete harmony with the UTC and CCDS.

Selleri had an independent mind and therefore he was not listened to that much. Selleri said that physics, like fashions in clothing, is dominated by fads, as defined by the tribe of physicists, who listen to other members of the tribe, but ignore what outsiders say. An idea that is fashionable and in vogue one year will vanish the next year, when physicists become infatuated with a new style of thinking. Their main interest is money and power, not truth. Therefore it is essential for academic physicists to keep up with the fads that are currently in vogue, which never included the issues that Selleri was interested in.

Selleri authored 350 scholarly papers and many books, but because his ideas were not mainstream, neither he nor his students ever achieved much notoriety. He believed theoretical physics cannot be driven by expert consensus, but only by empirical observations. The Italian tradition of atomic physics was established by Enrico Fermi, who built the first nuclear reactor, and who was strongly biased in favor of empirical research instead of expert opinion. Fermi disliked the Copenhagen interpretation. Fermi was 35 years older than Selleri.

\section{Selleri said, "History teaches that in science the majority is usually wrong." (35)}

If you study Selleri, it is important to know that he does not use the Lorentz factor (Eq. 4) in his discussions of relativity:

$$
\gamma=\frac{1}{\sqrt{1-\left(v^{2} / c^{2}\right)}}
$$

Instead, Selleri uses the inverse:

$$
R=\frac{1}{\gamma}=\sqrt{1-\left(v^{2} / c^{2}\right)}
$$

\subsection{Aether and absolute simultaneity}

Given that Sagnac demonstrated the existence of aether, and the CCDS endorsed Sagnac, what is aether? As a first approximation we can say that it is the vacuum of empty space within which there is absolute simultaneity.

To explain the history of aether we must go back a century and a half and talk about the speed of light. It was thought that light was a wave and they thought it must travel through some medium, the way water waves travel through water. The earth travels $30 \mathrm{~km} / \mathrm{sec}$ around the sun, and the direction reverses

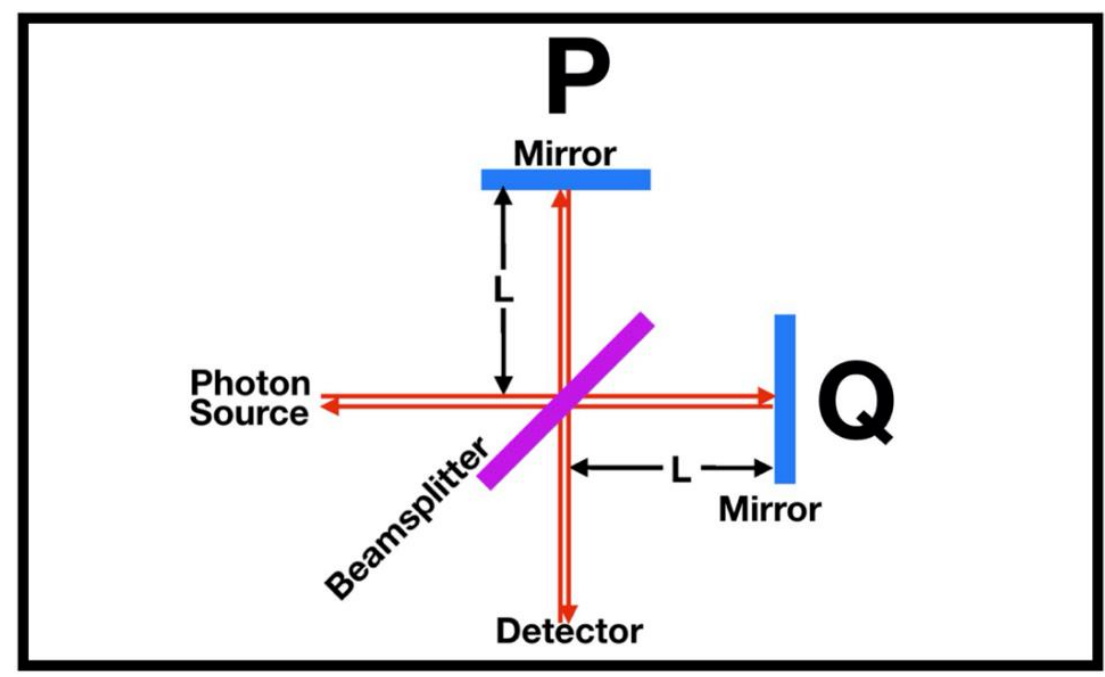


every six months. Therefore, if the earth travels through aether, the speed of the "wind of aether" should be different six months later.

Fig. 7. In 1881 Michelson's equipment measures only the two-way, not the one-way speed of light.

The interferometer was turned such that first the $P$ arm, then the $Q$ arm was aligned with motion of the earth through aether. If the 2-way speed of light was slower in one direction than in the orthogonal direction, there should be wave interference. But there was no such interference.

To measure the speed with which the earth passes through aether, Michelson and Morley used the contraption shown in Fig. 7 to measure the 2-way speed of light in all directions and found that it was always the same: no 6-month variation. The Trouton-Noble experiment in 1903 came to similar conclusions. (45)

The 2-way speed of light means going out from a source, hitting a mirror, and coming back. Remember what we said about microwaves going around the earth? If they put a mirror up and after the microwave went west it hit the mirror and went around the earth to the east, you would find that there is always the same 2-way speed of light. And you would not recognize that light goes faster to the west than to the east.

Selleri agrees with Lorentz in saying that because of the Lorentz transformations we can never directly measure the speed of the "wind of aether."

The Dutch physicist Hendrik Lorentz (1853-1928) studied how James Clerk Maxwell's equations could be modified to fit the Michelson-Morley data. Lorentz remained enthusiastic about aether until his death. Lorentz discovered that Maxwell's equations could be reconciled with the Michelson-Morley data with what we now call, "Lorentz invariance," by means of the following equations. (44)

$$
\begin{gathered}
\gamma=\frac{1}{\sqrt{1-\left(v^{2} / c^{2}\right)}} \\
x^{\prime}=\gamma(x-v t) \\
y^{\prime}=y \quad z^{\prime}=z \\
t^{\prime}=\gamma\left(t-\frac{v x}{c^{2}}\right) \\
m^{\prime}=\gamma m
\end{gathered}
$$

where $\boldsymbol{\gamma}$ is the "Lorentz factor" defined by Eq. $4, v$ is our velocity through the aether, and $x, y, z, t$ and $m$ are the three axes, time and mass.

Here is what the Lorentz transformations do. If a woman sitting in a chair in outer space is holding a glass jar with a light bouncing up and down, assume it is bouncing 5 times per second. As her chair accelerates to almost the speed of light, the rate of bouncing decreases to about one per second. In other words, time slows down as you approach the speed of light. This is called "time dilation." The length of her chair also shrinks which is called "length contraction." Also, as the woman approaches the speed of light, she and her chair become heavier and heavier.

Einstein adopted the Lorentz transformations as the essence of his special relativity. Einstein and Lorentz remained friends throughout their lives. But Lorentz always believed in the existence of aether. Einstein at first denied the existence of aether, and therefore got into all this trouble about time. By 1920 Einstein realized that without aether there would be no structure to empty space. Since his Theory of General Relativity described a structure to space, Einstein changed his mind and endorsed aether in 1920. (32)

\subsection{Einstein's strong relativity contrasted with Selleri's weak relativity}


Franco Selleri, whose viewpoint is adopted in the first half of this article, draws a distinction between strong and weak relativity. Einstein advocated strong relativity, which assumes or postulates that the laws of nature are the same in all inertial frames of reference, and there is no simultaneity. Selleri advocates weak relativity, which is to say that we can never directly measure our speed through aether. The Michelson-Morley experiments are correct. The reason we can't measure it is because of the Lorentz transformations.

Nevertheless, Selleri says there is a "preferred" frame of reference, a stationary frame of reference somewhere in the universe, which is the basis of our simultaneity. This is the same frame of reference that Isaac Newton postulated, although it is bent by gravity (as in General Relativity). It is in this frame of reference (shown in blue in Fig. 8) that Lorentzian aether at rest is anchored. Only in that stationary frame of reference is the one-way speed of light the same in all directions.

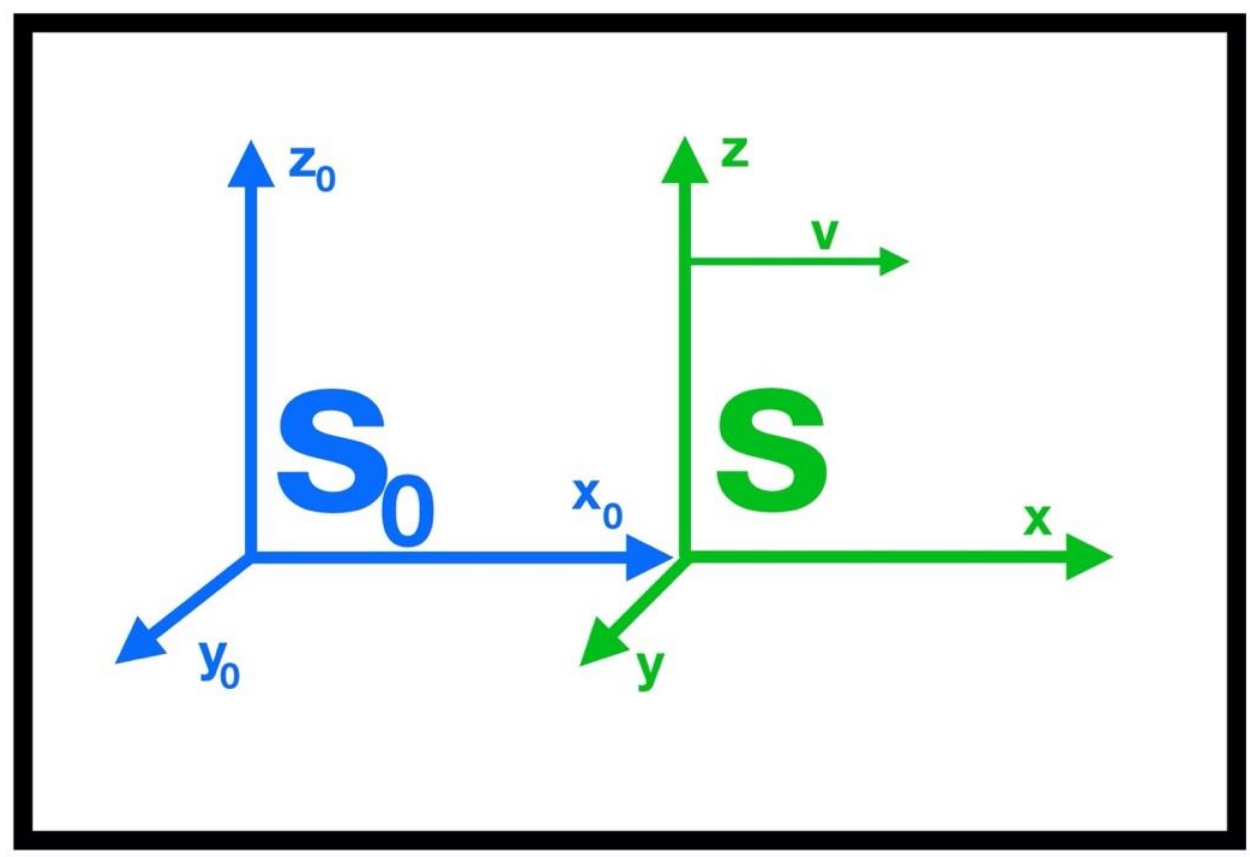

Fig. 8. Our inertial frame (S in green, on the right) is moving at velocity $v$ relative to the stationary inertial

frame $\boldsymbol{S}_{\mathbf{0}}$, which is where aether is anchored. The one-way speed of light $\boldsymbol{c}$ is the same in all directions only in $\mathbf{S}_{\mathbf{0}}$, not in $\mathbf{S}$. Therefore $\mathbf{S}_{\mathbf{0}}$ is called the "preferred inertial frame." Because of the Lorentzian transformations we can never directly measure $v$, but we can indirectly measure it at $630 \mathrm{~km} / \mathrm{sec}$, assuming the CMBR is stationary. 
The properties of $\mathbf{S}_{\mathbf{0}}$ are astonishing. When someone in $\mathbf{S}$ observes $\mathbf{S}_{\mathbf{0}}$, time in $\mathbf{S}_{\mathbf{0}}$ goes faster (antidilation of time) and a one meter ruler in $\mathbf{S}_{\mathbf{0}}$ is longer than one meter in $\mathbf{S}$. That is the reciprocal of an observer in $\mathbf{S}_{\mathbf{0}}$ who looks at $\mathbf{S}$, where there is time dilation and length contraction.

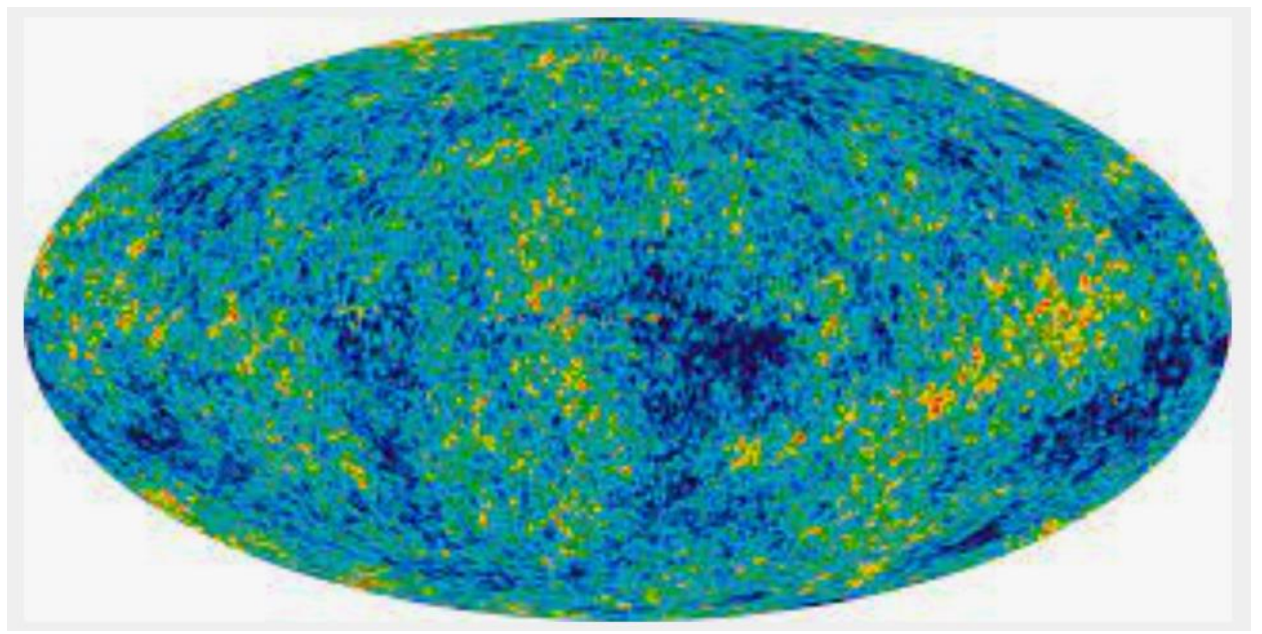

Fig. 9. We assume the "preferred" or stationary frame of reference $\mathbf{S}_{\mathbf{0}}$ is stationary relative to the CMBR

(Cosmic Microwave Background Radiation).

There are three issues in our discussion of relativity.

a) Because of the Lorentz transformation equations, there is no direct way to measure our speed through aether. Aether is anchored in a stationary frame of reference $\mathbf{S}_{\mathbf{0}}$.

b) There exists a stationary frame of reference $\mathbf{S}_{\mathbf{0}}$ somewhere in the universe, which is the only inertial frame where the one-way speed of light is the same in all directions.

c) That stationary frame is anchored in the Cosmic Microwave Background Radiation (CMBR).

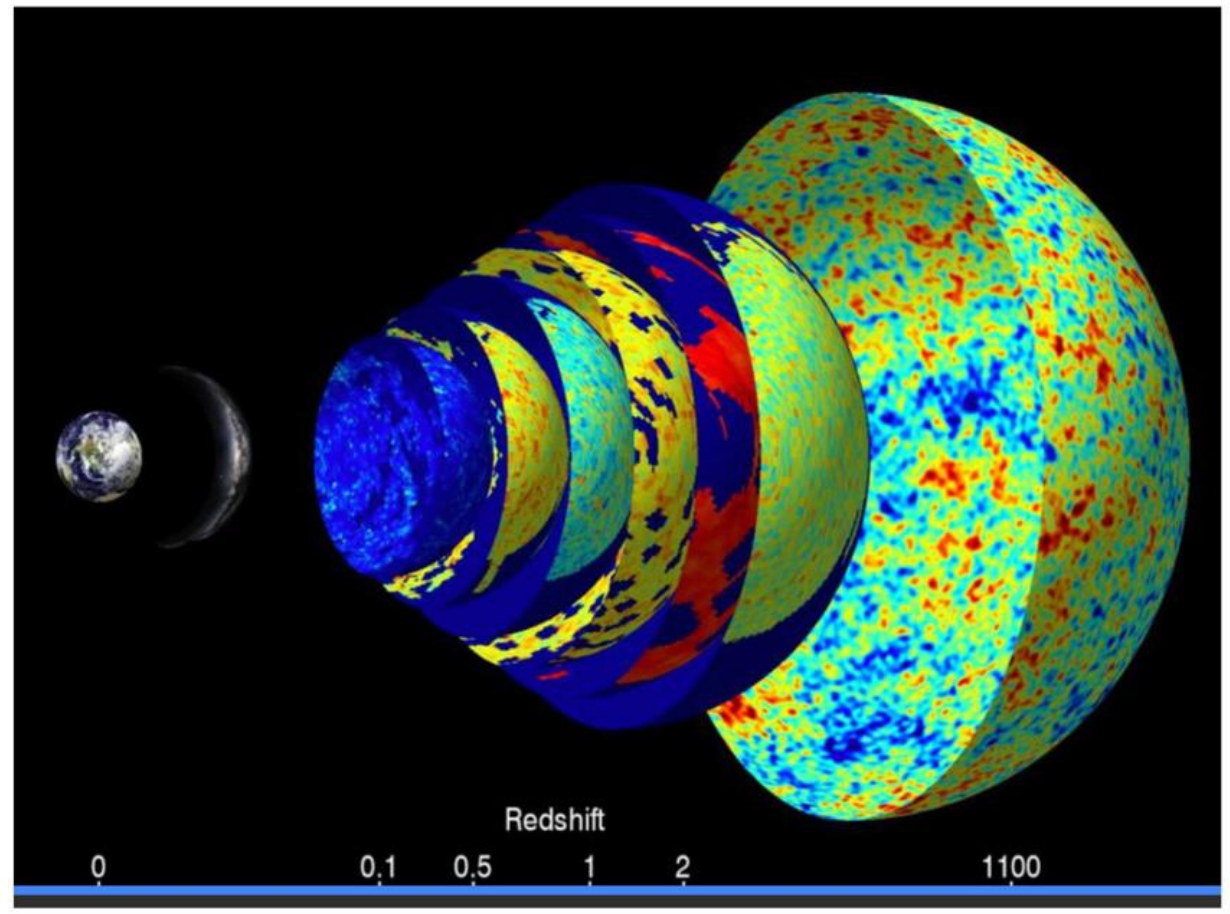


Fig. 10. This is a map of our location in the stationary aether of the universe.

We can approximate that stationary frame of reference if we assume that this $\mathbf{S}_{\mathbf{0}}$ frame of reference is not moving relative to the CMBR. We know from slight variations in the background radiation (anisotropy above and below $2.725^{\circ}$ Kelvin) that we are moving at about $630 \mathrm{~km} / \mathrm{sec}$ towards the constellation Leo, which is three times the speed of rotation of the Milky Way Galaxy. The constellation Leo is also moving at $630 \mathrm{~km}$ per second in the same direction that we are, so we are not getting any closer to Leo. We are both moving relative to the CMBR. Some scholars would say there is a "wind of aether" blowing past Leo and the earth, at velocity $630 \mathrm{~km} / \mathrm{sec}$. The speed of the "wind of aether" cannot be directly measured. It passes straight through us and through the earth.

\subsection{There is no space-time continuum}

Selleri discovered that Lorentz had made a mistake in his equation for time dilation. Time slows down with velocity and gravitation. But it does not slow down when you are further away. Lorentz's mistake was to indicate that time slows down when you are further away (see Eq. 7).

$$
t^{\prime}=\gamma\left(t-\frac{v \mathbf{X}}{c^{2}}\right)
$$

Lorentz inserted the variable " $x$ " in the upper right corner of Eq. 7, for no apparent reason. He never justified why he did that. Selleri says the dilation of time is caused by speed and gravity, but it is not caused by distance from the observer (" $x$ "). Here is Selleri's equation for time dilation:

$$
t^{\prime}=\gamma t
$$

(9)

This equation is endlessly confusing. It is easy to get muddled about which frame of reference has time $\boldsymbol{t}$ and which has time $\boldsymbol{t}^{\prime}$. The point is simple. Eq. 9 is intended to refer to time dilation with velocity or gravitation, but it omits the variable " $x$ " which is because time does not dilate with distance $(x)$.

Selleri points out that when you correct this equation of Lorentz's, there is no space-like quality to time. Therefore, there is no "space-time continuum." There is a 3-dimensional space continuum which is bent by gravity, but time is not like a fourth dimension of space.

Absolute simultaneity and the discarding of a 4-dimensional space-time continuum are two aspects of the same thing. Each one implies the other. When you remove a spatial component " $x$ " from Lorentz's time transformation, what results is absolute simultaneity. The opposite is also true. When you have absolute simultaneity (as in the CCDS) then you necessarily remove the spatial component " $x$ " from your calculation of time. In other words, the standardized time displayed on your cell phone contradicts the idea of a space-time continuum.

The result of that error on the part of Lorentz has been catastrophic, especially for Einstein's personal mental health. Einstein was incarcerated all his life in the Minkowski prison of hyper-determinism, as you will discover below. Minkowski hyper-determinism encourages passivity and despair as one's overall attitude toward life.

Hermann Minkowski (1864-1909) took Lorentz' error about time and built it into the Minkowski manifold, in which time is a dimension of space-time, as in this equation:

$$
c^{2} t^{2}-x^{2}-y^{2}-z^{2}=1
$$

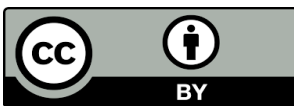


This leads to a kind of Pythagorean theorem for measuring distance in this alleged "space-time":

$$
\text { distance from } \mathrm{A} \text { to } \mathrm{B} \text { is }=\frac{1}{\gamma} \sqrt{\left(\sum_{i=1}^{3}\left(a_{i}-b_{i}\right)^{2}\right)-c^{2}\left(t_{A}-t_{B}\right)^{2}}
$$

There is no such thing as a space-time continuum. Like Selleri, we reject Eqs. 10 and 11. In 1905 Einstein made a mistake by adopting the Minkowski manifold, and its four-vectors. That mistaken use of fourvectors persisted in Einstein's Theory of General Relativity (TGR). (33)

If we could remove the four-vectors, and change them to three-vectors plus time, then Einstein's tensors of the Theory of General Relativity (TGR) would be correct.

$$
\begin{aligned}
& R(x, y ; u, v)=\left[\begin{array}{cc}
s(x, u) & s(x, v) \\
s(y, u) & s(y, v)
\end{array}\right] \\
& =\sum_{k=1}^{r}\left[\begin{array}{cc}
a_{i p}^{(k)} x_{i} u_{p} & a_{i q}^{(k)} x_{i} u_{q} \\
a_{j p}^{(k)} x_{j} u_{p} & a_{j q}^{(k)} x_{j} u_{q}
\end{array}\right]
\end{aligned}
$$

The study of the shape of non-Euclidean space is greatly enriched by the Calabi-Yau manifold with its Ricci-flat metric. Shing-Tung Yau has vastly enriched our understanding of the topology of TGR.

We will use your own experience to prove that there is no "space-time continuum." And then we will talk about why that is very psychologically important. If there were a "space-time continuum" and we're counting time in nanoseconds, then somewhere on earth there is a person in an airplane who is 10 nanoseconds ahead of you. We will exaggerate and say he is ten minutes ahead of you. So, that person can look over and see your life and see what's going to happen in the next 10 minutes.

You may feel that you are free to do anything. But that other person can see what's going to happen 3 minutes from now, at which time you are going to reach up and lightly scratch the righthand side of your face. So, when 3 minutes arrives, you don't have any choice about it. You must reach up and scratch the right side of your face because that other person has already seen it happen.

So, could that kind of hyper-determinism be true? Philosopher Karl Popper said in his autobiography that Einstein was trapped in such a Minkowski prison of hyper-determinism. But we know that it's not true. Why? Because of the CCDS. We know there is absolute simultaneity everywhere, and therefore the other person in the airplane cannot be 10 nanoseconds or 10 minutes ahead of you. He or she has precisely the same time as you! And that makes the idea of space-time continuum impossible.

So, to summarize, if you have a cell phone, then you are telling time by the Coordinated Universal Time (UTC), and the UTC system is based on the concept of absolute simultaneity, which means that all other observers anywhere have precisely the same UTC time and the same sequence of events as you do. Which means there cannot be any space-time continuum.

\subsection{Mathematics alone is not enough}

We live in a scientific culture where physicists tend to trust equations if the equations are verified by empirical data. One of Selleri's central ideas is that equations alone don't tell the whole story. Here is what he wrote: "One should never forget that behind the equations of a theory there is a huge qualitative structure made of empirical results, generalizations, hypotheses, philosophical choices, historical conditionings, personal tastes, conveniences.... The correctness of the mathematical formalism is not enough to validate a scientific structure."

Here is an example. If you limit your focus to the equations of TSR you might convince yourself that "Einstein's TSR is correct" because there is a mountain of empirical data that says that the round-trip speed of light in a vacuum is $299,792,458 \mathrm{~m} / \mathrm{sec}$ for all observers. But Einstein paid a high price to 
purchase his special relativity: the loss of simultaneity. Furthermore the round-trip and one-way speed of light are not the same thing.

Many, many physics experts have gone down the rabbit hole with Einstein in his 1905 theory of special relativity, in which he was so in love with the idea of the speed of light being the same for all observers, that he was quite willing to sacrifice simultaneity. In the 21st century that viewpoint is impossible to maintain, unless you avoid all cell phones and computer networks.

The bottom-line message of this entire article is that you need common-sense. Mathematics cannot replace common-sense. Often mountains of mathematical equations are used for the purpose of hiding the absence of common-sense. Mathematics is improperly used if it is used for the purpose of obscuring reality.

\section{Common-sense about quantum weirdness}

We turn now to the second half of this article: common-sense about quantum weirdness. This is a subject about which we claim, with good reason, physicists have lost their common-sense. $(1,2,4-31,46)$

Quantum mechanics is the science of atoms and subatomic particles. There are ten-billion-billion atoms in every grain of sand. Although we can probe the subatomic world with experiments and quantum mathematics, it appears to be organized in a way that derails our thinking, prior to this article. This article is the first time that a coherent picture of the quantum world has been presented.

We claim that nature is organized in a dramatically different way than what scientists envision. Physicists are able to predict the behaviour of the quantum world by means of quantum mathematics, but they are not able to formulate a coherent picture of what the quantum world is like. Below we will present the first ever coherent picture that corresponds to both quantum experiments AND quantum mathematics.

But there are two problems with the picture we are about to present. The first is that it is a century too late in arriving. If this proposal had been made in the 1920's when physicists were searching for such a coherent picture, it would probably have been applauded. Today the physics community greets such a solution with apathy or hostility.

During the past century physicists have been defeated by quantum weirdness. They act like people who have been defeated in war, and who have learned to adjust their thinking so as to avoid "dangerous" subjects.

There is a glaring difference between the culture of physicists and that of mathematicians. After a century of defeat, physicists are willing to say that a problem cannot be solved, so it is expedient to avoid thinking about it. Mathematicians see an insoluble problem as an exciting possibility. Waiting 350 years for Fermat's last theorem or 160 years for the Riemann hypothesis makes the problem a more attractive prize. But physicists do NOT yearn for the day when they will solve quantum weirdness. They have come to believe that it is best to avoid thinking about it.

Unfortunately, scientists have lost the ability to think. That sounds arrogant, to say that brilliant people, some of whom have Nobel Prizes, have lost the ability to think. It is a consequence of physicists losing the desire or motivation to ponder such a frustrating problem as quantum weirdness, and therefore refusing to use their brilliant minds to grapple with TEW (Theory of Elementary Waves). If scientists would toggle the switch on the side of their skull to "ON," they would be able to think. But they have become lazy and avoidant of this subject. There is no money or prestige in it. So why bother?

For example, this author's college roommate subsequently worked for half a century on the faculty of the Jet Propulsion Labs at the California Institute of Technology studying solar wind. When we solicited his comments on TEW, he replied, "I have zero interest in quantum weirdness. I don't want to waste time discussing the double slit experiment. I have more important things to do, like riding bicycles with my son." 
Since quantum weirdness was a problem that defeated Einstein and many of the greatest geniuses of all time, who spent decades, and were unable to solve the problem, we might expect that when a solution finally emerges (as it will below) the quantum world will look drastically different than anything we expected. Christopher Zeeman used to say "It is a mistake to read the research before tackling an insoluble mathematics problem, because it will slot your mind into the same groove everyone else is trapped in."

What you are about to read is outside the groove in which everyone else is trapped. For example, when people hear that we reject wave-particle duality (see Section 3.6), most physicists stop reading. That is because most physicists are trapped in the groove that hides the quantum world from view.

Thomas Kuhn's book, The Structure of Scientific Revolutions, says that a revolution in scientific thinking constitutes a paradigm shift, and leaders of science, trained in the obsolete paradigm, invariably find the new paradigm to be unintelligible gibberish. (38)

One example of a paradigm shift was Alfred Wegener's proposal of continental drift. In 1912 Wegener proposed that there had once been a super-continent which he named "Pangaea" that had broken apart, and all of today's continents were pieces that drifted to their current position. His idea was universally rejected by all leading scientists and scholarly societies, as too preposterous to be discussed, because it was obvious to all thinking people that there was no force strong enough to move continents across the face of the earth. For the next half-century, Pangaea was never mentioned in science. (65)

A paradigm shift is an exciting opportunity for young scientists to make a career. It is akin to discovering a new, unexplored continent, and having the opportunity to draw the first maps.

\subsection{The central riddle of QM}

Here is a riddle. In the next dozen equations, we will develop the Schrödinger equation. Yet something is missing. The question is: what is missing?

How does a quantum particle travel from the particle source to a detector, such as the human eye? We will describe the motion using a simple plane wave

$$
\psi_{R}(x, t)=e^{i(k x-\omega t)}
$$

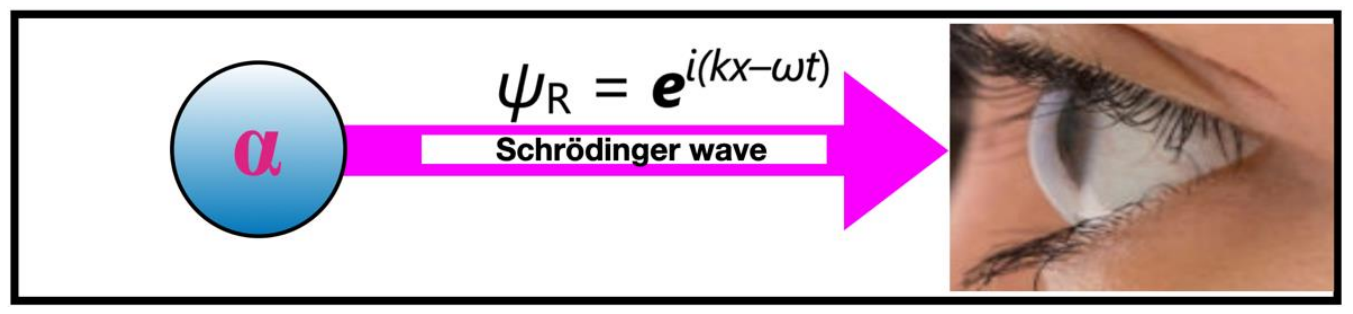

Fig. 11. How does a plane wave develop into a Schrödinger wave?

We use the subscript $\mathbf{R}$ in our wave equation because the plane wave is moving to the Right. Using that plane wave, we now focus on energy as we develop the Schrödinger equation:

$$
\begin{gathered}
E=\text { kinetic energy }+ \text { potential energy } \\
E=\frac{1}{2} m v^{2}+u=\frac{p^{2}}{2 m}+u
\end{gathered}
$$

Taking the second derivative $\partial^{2} / \partial x^{2}$ of the wave function $\psi_{R}=e^{i(k x-\omega t)}$ (Eq. 14) we get:

$$
\begin{array}{r}
\frac{\partial^{2} \psi_{R}}{\partial x^{2}}=\frac{\partial^{2}}{\partial x^{2}}\left(e^{i(k x-\omega t)}\right)=(i k)^{2} \psi_{R}=- \\
k^{2} \psi_{R}=\frac{p^{2}}{\hbar^{2}} \psi_{R} \\
\hbar^{2} \frac{\partial^{2} \psi_{R}}{\partial x^{2}}=p^{2} \psi_{R}
\end{array}
$$


Multiplying both sides of Eq. 16, $\left[E=\left(p^{2} / 2 m\right)+u\right]$, by $\psi_{R}$, we get:

$$
E \psi_{R}=\frac{p^{2} \psi_{R}}{2 m}+u \psi_{R}
$$

and inserting Eq. 18 we get the Time Independent Schrödinger Equation (TISE):

$$
E \psi_{R}=-\frac{\hbar^{2}}{2 m} \frac{\partial^{2} \psi_{R}}{\partial x^{2}}+u \psi_{R}=\mathbf{T I S E}
$$

The time dependent equation can be easily derived by differentiating our wave equation

$$
\begin{gathered}
\psi_{R}=e^{i(k x-\omega t)} \quad \text { by } \partial / \partial t: \\
\frac{\partial \psi_{R}}{\partial t}=-i \omega \psi_{R}
\end{gathered}
$$

We define $E=\hbar \omega$. Multiplying that by $\psi_{R}$ we get:

$$
\begin{array}{r}
E \psi_{R}=\hbar \omega \psi_{R} \\
-\frac{i}{\hbar} E \psi_{R}=-i \omega \psi_{R}=\frac{\partial \psi_{R}}{\partial t} \\
E \psi_{R}=-\frac{\hbar}{i} \frac{\partial \psi_{R}}{\partial t}=i \hbar \frac{\partial \psi_{R}}{\partial t}
\end{array}
$$

We can substitute that into the TISE and that gives us:

$$
i \hbar \frac{\partial \psi_{R}}{\partial t}=-\frac{\hbar^{2}}{2 m} \frac{\partial^{2} \psi_{R}}{\partial x^{2}}+u \psi_{R}
$$

Which is the Time Dependent Schrödinger Equation, TDSE.

Can you see what the problem is? Ever since Erwin Schrödinger derived his wave equations in 192526 it has presented scientists with two problems:

1. It is the most important equation in quantum physics.

2. It covers everything, and yet, somehow, it leaves something out. What is missing?

The answer (Fig. 12) was given by Richard Feynman, who said, "Quantum particles behave differently if we watch them than if we close our eyes."

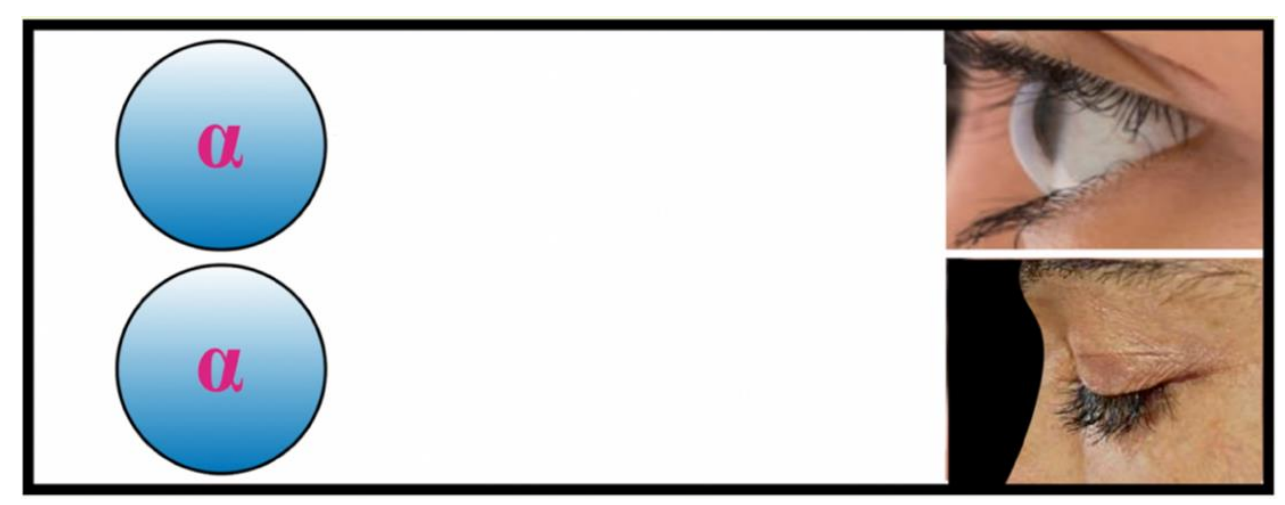

Fig. 12. Feynman says a quantum particle ( $\boldsymbol{\alpha})$ behaves differently if we are watching it.

How can the Schrödinger wave account for this peculiar idea? We need a new perspective, to preserve the Schrödinger equation intact, yet explain why it makes a difference whether humans are watching.

\subsection{A proposed model to solve the riddle}

We propose that every Schrödinger wave has a pre-history. Before the particle is emitted by the source, nature is bursting with excitement about what might happen next. We propose that a covert signal (Fig. 
13) emanates from the human eye (or from any detector) and strikes the particle before the particle takes flight back to the human eye.

The blue arrow in Fig. 13 represents a zero-energy wave. A lot of people get hung up right there. They say, "There's no such thing as zero-energy waves! Every wave carries energy." Well, that's not true, even though it is widely taught. (49) The Schrödinger wave for example, does not carry energy. It carries something called a Hamiltonian operator or a momentum operator. But it carries no raw energy. The Schrödinger wave cannot push or pull particles, it can do no work. It describes what might happen but does not propel things to happen in that way. That's because it does not carry any energy.

That is typical of waves in the quantum world. They carry probability amplitudes, but they don't carry energy. (3) So, the waves we're talking about in Fig. 13 are like all the other waves, like the Schrödinger wave in that it is a zero-energy wave. We're talking about two of them. One going out from the eye to the particle. And then the Schrödinger wave carrying the particle back to the eye.

We will call the zero-energy wave that goes out from your eye, an "Elementary Wave." Our best plan is to build a model in the simplest possible way. So, let's just consider a one-dimensional wave. Now such a wave when it hits a vertical barrier tends to reflect. It bounces off the wall and goes back in the opposite direction from which it came. In nature one often finds waves that reverse directions if they hit an orthogonal obstacle. Remember that we are building a hypothetical model.

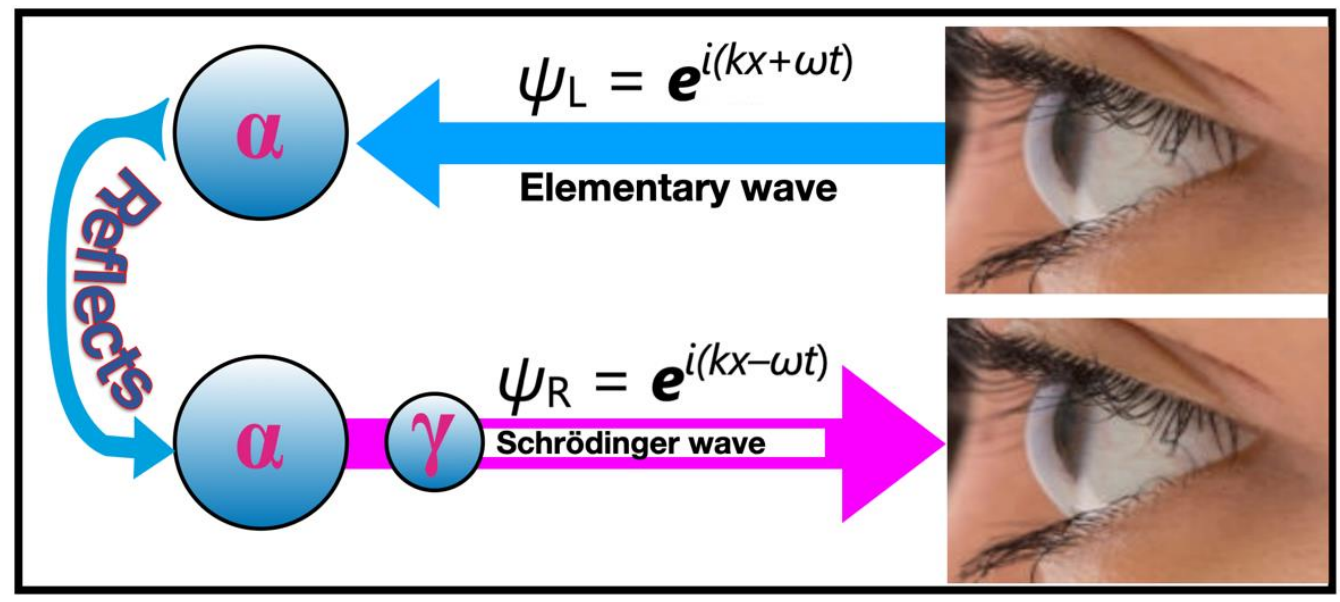

Fig. 13. This is simplest model we can propose, in one-dimension. The Schrödinger wave (in pink) carries particle gamma (a photon) to the detector.

Two solutions of the wave equation $\left(\mathbf{u}_{\mathbf{t t}}=\mathbf{c}^{2} \mathbf{u}_{\mathbf{x x}}\right)$ are $u=\boldsymbol{e}^{i(k x+\omega t)}$ and $u=\boldsymbol{e}^{i(x k-\omega t)}$, representing a wave moving to the left $\left(\psi_{\mathrm{L}}=\boldsymbol{e}^{i(k x+\omega t)}\right)$ and right $\left(\psi_{\mathrm{R}}=\boldsymbol{e}^{i(x k-\omega t)}\right)$. Above we showed how the rightward moving wave can blossom into a Schrödinger wave, still a zero energy wave. However, the mathematical manoeuvres we used to allow $\psi_{\mathrm{R}}$ to blossom will not work to make $\psi_{\mathrm{L}}$ blossom. There is a plus sign in the exponent which prevents the Schrödinger wave from developing from $\psi_{\mathrm{L}}$.

From now on we are going to refer to this complicated mechanism (Fig. 13) by the following six words, "Particles follow zero-energy Elementary Waves backwards."

\section{Particles follow zero-energy Elementary Waves backwards.}

We say that because, if you overlook the details, first an Elementary Wave goes out from the detector, and the result is that a particle $(\boldsymbol{\gamma})$ follows that trajectory back to the detector. 
That is a lot of baggage to put on that wave and particle. What would make us think that particles and waves would behave in such a peculiar manner in the quantum world? We would not believe it if we had not spent the last ten years reviewing a mountain of published experimental evidence, which says that particles do follow zero-energy Elementary Waves backwards. (4-31)

However, our model (Fig. 14) is slightly more complicated than what we just stated.

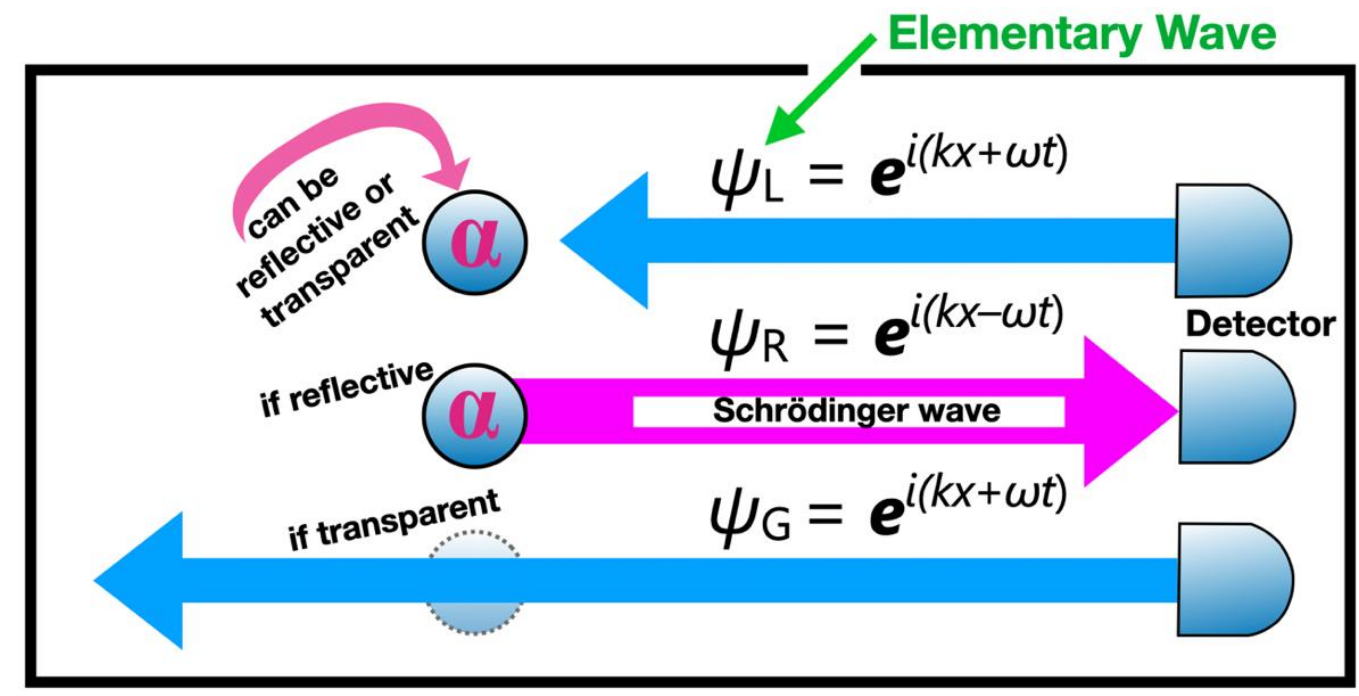

Fig. 14. This shows our full model. The particle $\boldsymbol{\alpha}$ can exist in two states (reflective or transparent). If it is transparent then $\psi_{\mathrm{L}}$ passes straight through and vanishes off the horizon to the left, becoming $\psi_{\mathrm{G}}$. The subscript " $G$ " in " $\psi_{\mathrm{G}}$ " means "ghost," and the ratio of Elementary Waves in this category to $\psi_{\mathrm{L}}$ waves is infinity to one.

In any volume of space there are a finite number of particles (or a finite amount of energy) but an infinite number of waves $\psi_{\mathrm{G}}$ with no particle attached. Therefore the ratio of $\psi_{\mathrm{G}}$ to $\psi_{\mathrm{R}}$ is infinity to one, and it is a high order of infinity.

Note that our model applies only to free particles, not particles that are bound in atoms or molecules or harmonic oscillators. The reason is that if a particle is gyrating in ellipses, it doesn't really matter much whether a particle is following a wave backwards or forwards, or whether the particle is itself the wave. So, TEW does not differ significantly from QM except for free particles.

Focusing on our central model, Fig. 13, we now ask this question: when does wave-function collapse occur? Below in section 4.3 we will define "wave-function collapse." In our model it occurs when the particle leaves the gun, carried by the Schrödinger wave. Prior to that the model is probabilistic. If there are Elementary Waves impinging on the particle from many directions, the particle will randomly select which incident Elementary Wave to respond to (i.e. the particle will select one incoming Elementary Wave to follow backwards) influenced by the amplitude with which that incident wave presents itself to the particle. But it is a random choice.

If we ask, "What controls the system?", the answer is that it is the interaction of the particle and Elementary Waves, an interaction that is often random.

As the particle makes that choice and leaves the gun in response to one incoming Elementary Wave, it becomes a deterministic experiment, with the particle following backwards that specific Elementary Ray with a probability of one, the same pathway that the Elementary Wave followed as it came from the detector to the particle gun. 
Wave-function collapse in our model occurs earlier, and at a different location, than in QM. In QM wavefunction collapse is said to occur when an observation is made, which is long after the particle left the gun. We will explore these issues in greater detail below: Section 4.3 .

\section{3 "Plain waves" but no "plane waves"}

Although we use the term "plane wave", it is of vital importance to recognize that our elementary waves never form parallel planes (Fig. 15).

Our wave equation ( $\mathbf{u}_{\mathbf{t t}}=\mathbf{c}^{\mathbf{2}} \mathbf{u}_{\mathbf{x x}}$ ) is a linear partial differential equation, and therefore any two solutions can be added together in a linear way and arrive at another solution. However, that only applies to the wave equation arising from one specific point of origin $\mathrm{x}_{0}$. Instead of the detector being the human eye, think of the detector as being a broad screen, as in the double slit experiment (which we will discuss in Section 4.2).

Other points of origin in proximity to $x_{0}$, points such as $x_{1}, x_{2}, x_{3}, \ldots x_{n}$, all have their own separate wave equations. Therefore the wave arising from one point cannot be added to the wave arising from another point. Therefore elementary waves are not "plane waves" in the sense of Fig. 15. We only speak of "plane waves" because the mathematical tradition is to call $\left(\psi=\boldsymbol{e}^{i(k x \pm \omega t)}\right)$ a "plane wave." It would be less confusing if we changed the spelling and called them "plain waves."

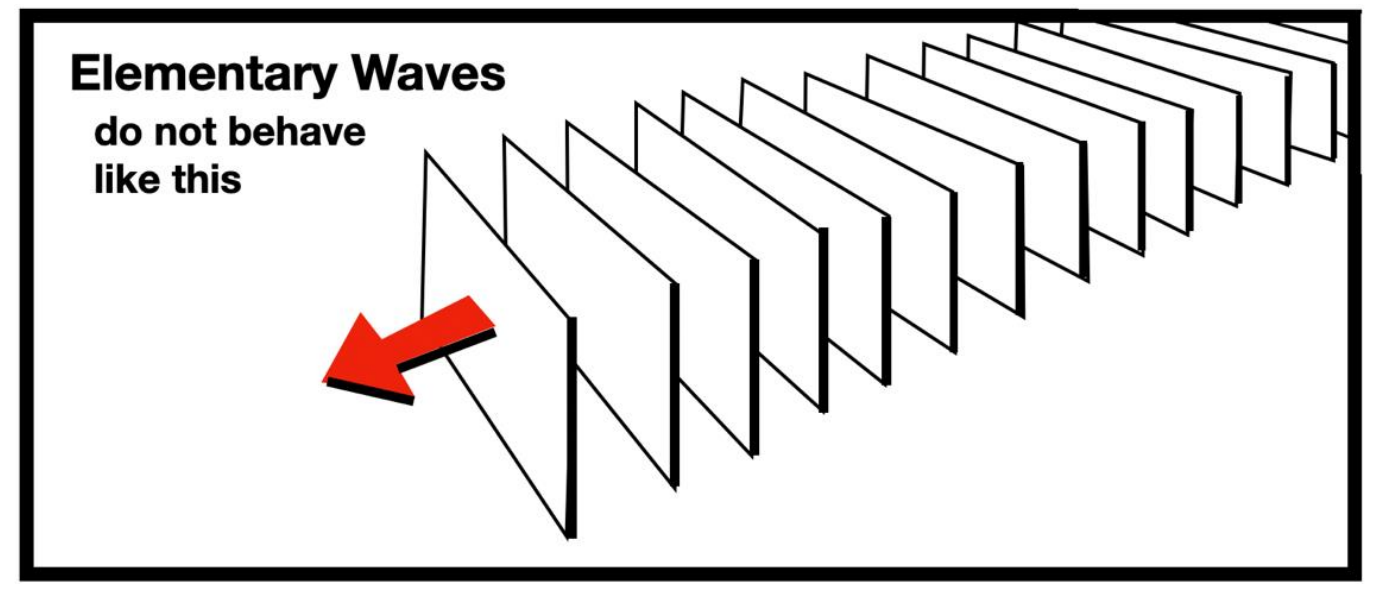

Fig. 15. When we use "plane wave equations" we are never referring to parallel planes like this. It would be less confusing if we changed the spelling and called ours, "plain wave equations."

What we just said about "no plane waves" explains why there is complementarity in the double slit experiment, as we will show below (Section 4.2.1). That is one of the deepest mysteries of QM that we will solve below.

Unlike QM, TEW does not involve the concept of a superposition of a particle. Particles in TEW have a definite location and velocity, limited by the Heisenberg Uncertainty Principle. Waves of course can be in a superposition in TEW. Since QM thinks of particles as being wave-packets, they can be in a superposition in QM.

Erwin Schrödinger, by the way, did not believe in the existence of physical particles. He thought they could be replaced by wave-packets. We don't agree. Our radical point of view is that we believe in the existence of the physical world, including physical particles. A physical particle, quantum particle, is carried in a wave-packet as its vehicle or container.

In TEW there is a distinction between the wave-packet and the physical particle. Only the wave can be in a superposition. For example, in a double slit experiment the particle goes through one and only one of the two slits, in TEW, as discussed below. 
Ours is not an "interpretation" of quantum math. Ours is a new mathematics. It is the mathematics of a hypothetical world called "The Elementary Wave World." We enfold the Schrödinger equation within other equations that change the meaning and behavior of a Schrödinger equation. You will be surprised at how different our Schrödinger wave equation behaves, than what you would expect.

One difference between TEW and QM concerns when we should start the stopwatch to time quantum experiments. This difference between QM and TEW concerning when the stopwatch begins, is a dramatic difference when we investigate the Bell test experiments below (in Section 4.5). The TEW picture of what is happening often violates the "light cones" that are published in QM scholarly articles about Bell test experiments. In other words, from a QM viewpoint the Elementary Waves would be classified as travelling faster than the speed of light. But from a TEW viewpoint we never exceed lightspeed. We simply start the stopwatch when the Elementary Waves begin to move, which is invariably long before any particle gets involved.

The Schrödinger equation acts differently in TEW than in QM in another respect. If a Schrödinger wave carries the particle to a detector, that same detector was the origin (earlier in time) of the Elementary Wave that later reflected and became a Schrödinger wave. It is like an elastic band connecting point " $z$ " (the detector) to the particle ( $\gamma$ or $\alpha$ ). As the Elementary Wave $\psi_{\mathrm{L}}$ from " $z$ " stretches the elastic band out to the particle, then the wave morphs into a Schrödinger wave $\psi_{\mathrm{R}}$ following the elastic band back to point " $z$." Thus " $z$ " is the origin and the destiny of the wave.

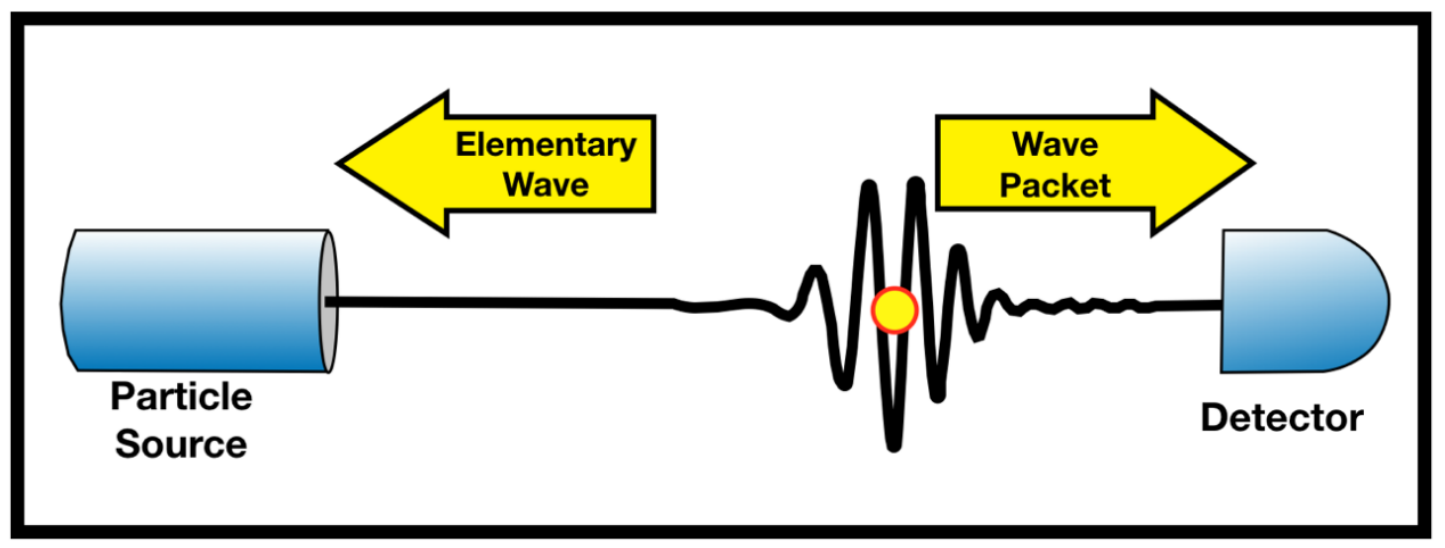

Fig. 16. An Elementary Wave travels from detector to the particle source, and a Schrödinger wavepacket carries the physical particle back to the detector.

\subsection{Does the moon only exist when people look at it?}

David Mermin, a professor of quantum physics at Cornell University, said, "Quantum mechanics has proved that the moon only exists when people look at it." (46) QM assumes that wave-function collapse occurs only when we observe something. This is the philosophical school of positivism, which had a huge impact on QM in Copenhagen in the early years of QM.

How does our model differ from David Mermin's comment about the moon?

The difference is that we propose that Elementary Waves perpetually come from everywhere, from all directions and at all wavelengths. There is nothing special about the human eye. If no one were looking at the moon it would continue to exist because the impinging Elementary Waves are always hitting it from every angle. Unlike Mermin's comment, our model is consistent with the idea that the moon is 4 billion years old. It existed before there were any multicellular life on earth to observe it.

\subsection{The relationship of "waves" and "quantum particles"}


Consider the relationship between waves and particles. QM says that they are two aspects of the same thing. Wave particle duality means that something in the quantum world can appear to be a wave if we look at it from one perspective or can appear to be a particle if we look at it from another perspective.

Another possible relationship of waves and particles is the "pilot wave" theory of Louise de Broglie and David Bohm. According to that idea there are zero-energy waves and particles follow those waves in the same direction as the waves are travelling. Franco Selleri subscribed to that idea. We reject the pilot wave theory for many reasons. We reject its determinism, its use of a "guiding equation", and we say that the pilot waves are travelling in the wrong direction.

Our viewpoint is that there are always zero-energy waves in nature and quantum particles follow those waves in the opposite direction. Below we will develop the partial differential wave equations to show how that could happen.

\subsection{History of the wave-particle duality error}

Below we will propose a mathematics that gets rid of all quantum weirdness. Since our model rejects wave-particle duality, let's discuss that.

How did physics arrive at the belief in the false doctrine of wave-particle duality? The history is simple. Louis de Broglie wrote a PhD thesis in 1924 in which he postulated that electrons and all matter had associated waves that obeyed some of the equations that Einstein had proposed for photons. (34)

$$
\lambda=h p=h m v
$$

That thesis fell into the hands of Erwin Schrödinger, who was steeped in the wave equations of classical physics:

$$
\begin{aligned}
\frac{\partial^{2} u}{\partial t^{2}} & =c^{2} \frac{\partial^{2} u}{\partial x^{2}} \\
u_{t t} & =c^{2} u_{x x}
\end{aligned}
$$

In discussing de Broglie's thesis with friends, Schrödinger realized that you can't talk about "waves" without a wave equation, and, using the de Broglie wavelength he developed the Schrödinger wave equation in 1925-1926. Schrödinger did not believe in the existence of physical particles. He thought they could be replaced by wave-packets, which is consistent with a Fourier transform. $(54,55)$

The idea of "wave-particle duality" is intrinsic to Schrödinger's idea that physical particles don't exist, that they can be replaced by wave-packets.

In 1927-1928 Clinton Davisson and Lester Germer at the Bell Labs discovered that there were wavelike properties associated with electrons as they ricocheted off (or refracted through) a crystal of nickel. This was immediately embraced by the founders of QM as confirmation of Schrödinger's idea that particles were actually wave-packets obeying wave dynamics. There have been many other experiments that confirm a statistical correlation between waves and particles, and those experiments has been considered "proof" of wave-particle duality.

When a quantum expert says that the doctrine of "wave-particle duality has been proved by empirical evidence," that means that the expert is unfamiliar with the Pfleegor and Mandel optics experiments of 1967-68, which disprove wave-particle duality. (14)

Pfleegor and Mandel constructed an experiment parallel to a double slit experiment. In a double slit experiment photons go through two slits and interfere with one another. Pfleegor and Mandel replaced the two slits with two lasers. Each individual photon must come from one laser or the other but not both simultaneously. They found a wave interference pattern on the target screen, and that pattern persisted as they reduced the frequency with which a photon was entering the equipment. They would have one photon go through the equipment, followed by 100 times as much time with no photon. There were 
never two photons in the equipment at the same time. Even with no photons, there continued to be an interference fringe pattern on the target screen.

Unlike a double slit experiment, one could not say the persistent interference wave pattern was caused by the same photon coming through both slits simultaneously. The only possible explanation of the wave pattern was that zero-energy waves were associated with each of the lasers, causing an interference pattern, and on those rare occasions when a photon was present, the photon made apparent the interference pattern that was already present. Those experiments were incompatible with wave-particle duality. (14)

\section{Testing our model vis-à-vis five quantum experiments}

So far we have built a model to which we gave the peculiar name, "Particles follow zero-energy Elementary Waves backwards." As absurd as our model appears at first to be, it is no more absurd and preposterous than the boxy contraption built by two bicycle mechanics, Wilbur and Orville Wright, a harebrained gadget that carried the two brothers aloft in 1903 at Kitty Hawk. First they built a glider in 1902 then added an engine in 1903. Their model (Fig. 17) did actually fly off the ground. It was the first airplane to ever do so. And it sparked a revolution.

The Wright brother's model is an analogy. Our quantum model looks as preposterous in the quantum world as did the Wright brothers model in the world of flight. Whether or not these models look preposterous is irrelevant. The only important issue is whether they can fly. Just as the Wright brothers' contraption could fly, we will use five quantum experiments to show that our model explains those experiments better than any other theory can do: much better.

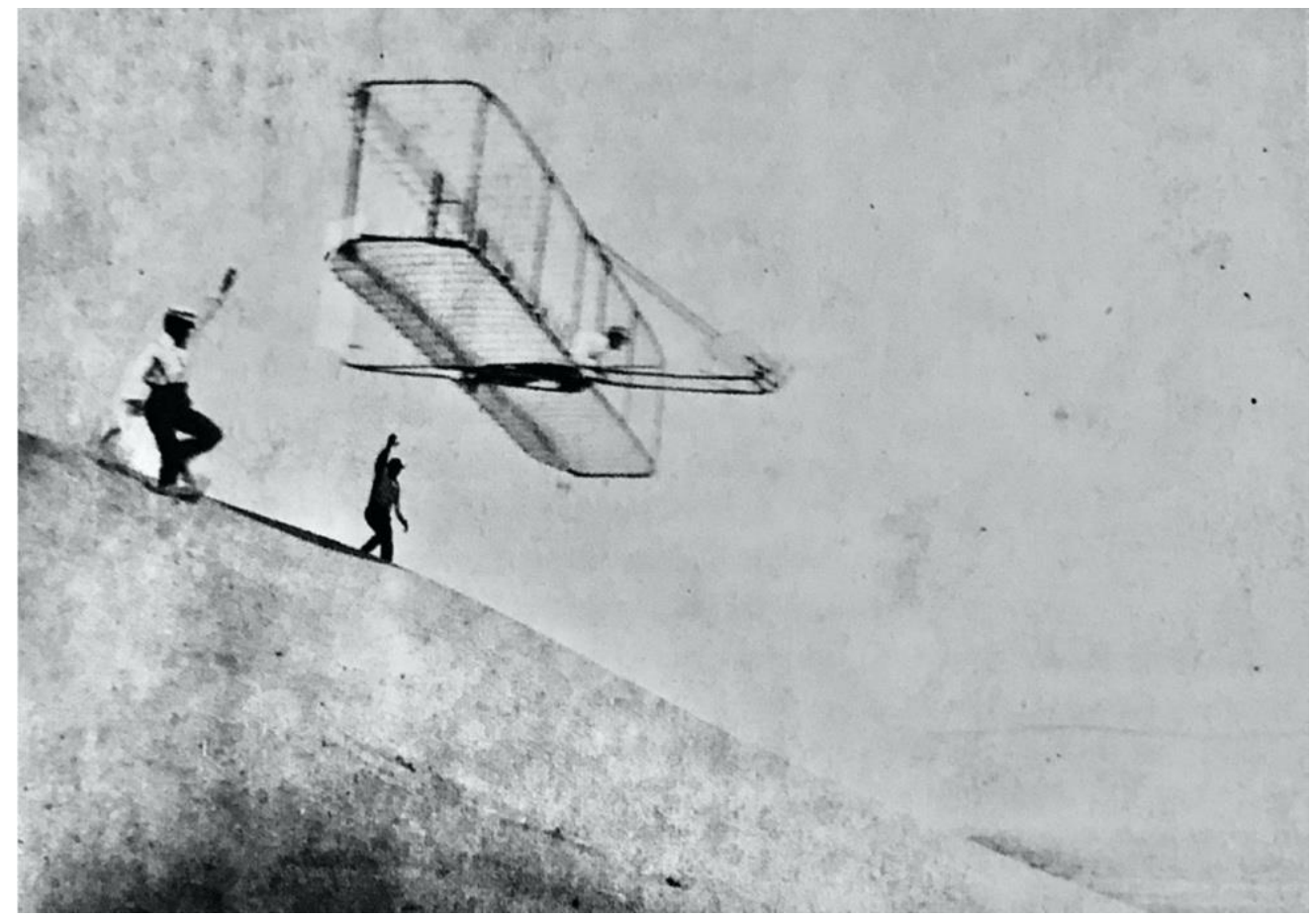

Fig. 17. In 1902 the Wright brothers built a kite, which they changed into a glider, which they flew at Kitty Hawk, a treeless barrier island off the Atlantic coast of North Carolina, USA. 


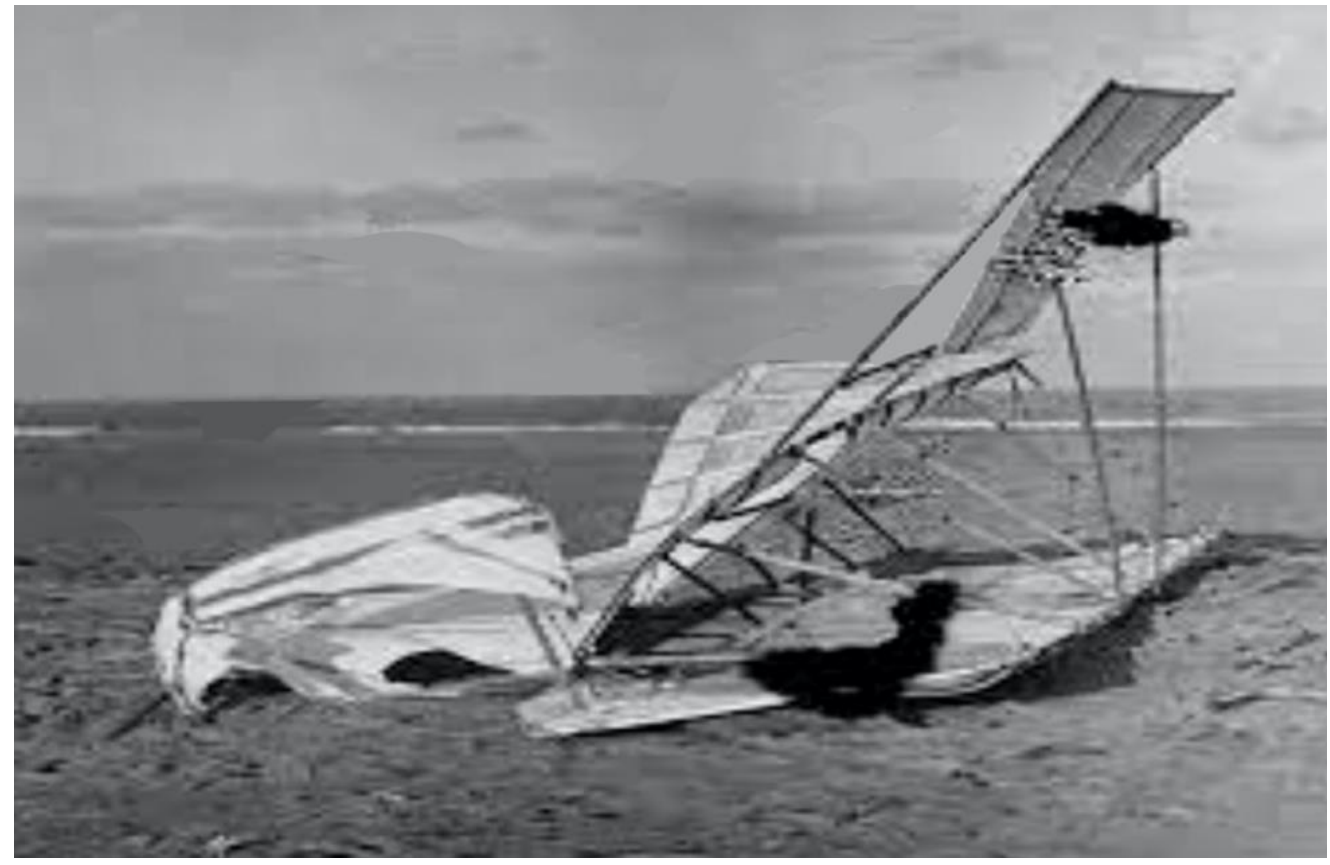

Fig. 18. Testing the first airplane was dangerous.

In 1903 they added a motor to their glider of 1902, and it became the first airplane. This is an analogy for the unlikely contraption we built as our model to explain the quantum world. It does not matter whether an experimental model looks absurd. The only thing that is relevant is whether it can fly. We will test our model against five experiments.

In 1908 a propeller broke and shredded the wings. Orville Wright fell 100 feet out of the sky, broke his leg, hips and ribs and spent seven weeks in the hospital. The passenger flying with Orville died. Meanwhile Wilbur flew 100 times in Europe, breaking every record for altitude, speed, and duration and attracting international attention.

The question now is whether or not our model will fly. We will test how it performs in five quantum experiments:

4.1 A neutron interferometer experiment

4.2 A double slit experiment with complementarity

4.3 The Schrödinger cat paradox

4.4 The Purcell effect

\subsection{A Bell test experiment}

These five experiments are like Kitty Hawk, where the Wright brothers tested their airplane (Fig. 11). In other words, if our model, as weird as it appears to be, can make sense of these five experiments, then we will proclaim that it has passed the initial tests and should be taken seriously, no matter how absurd it appears to be.

\subsection{A neutron interferometer experiment}

Consider first a neutron interferometer, that illustrates what we are saying about particles following waves backwards, and then, later in this article we will present our explanation of the Bell test experiments, which differs from Einstein's local realism, but also differs from QM. We have a much more practical and believable view of the Bell test experiments than did John Bell. 
The experiment was published in published in 1992 in Physical Review A by Kaiser, Clothier, Werner, et. al. "Coherence and spectral filtering in neutron interferometry," vol. 45, pp. 31-42, 1992. $(37,66)$ DOI: 10.1103/PhysRevA.45.31

Helmut Rauch in Vienna, Austria, established a research team that laid the foundations of neutron interferometry research. Their experimental work was well known and highly respected. They published many articles in leading journals of physics, including the article we are about to discuss.

Neutrons entered an interferometer where they bounced off various silicon blades. The blades divided the neutrons into two streams, $\psi_{1}$ and $\psi_{2}$, then the beams were recombined before they left the interferometer and went to a detector (Fig. 19). Where the streams divided there was an oscillating aluminum plate that changed the phase of $\psi_{2}$ compared to the phase of $\psi_{1}$, so the detector saw an interference pattern (a sine wave). They put in the upper beam $\left(\psi_{2}\right)$ a sample of bismuth of varying thickness. Bismuth slows down neutrons and neutron waves. When they increased the thickness of bismuth the amount of interference diminished, to the point that at $12 \mathrm{~mm}$ or more, all interference was gone (flat sine waves). They reasoned that the upper wave-packet $\left(\psi_{2}\right)$ had been so delayed that the lower wave-packet $\left(\psi_{1}\right)$ had already left the interferometer before the upper wave-packet arrived at the reunion point. That explained why interference diminished to zero.

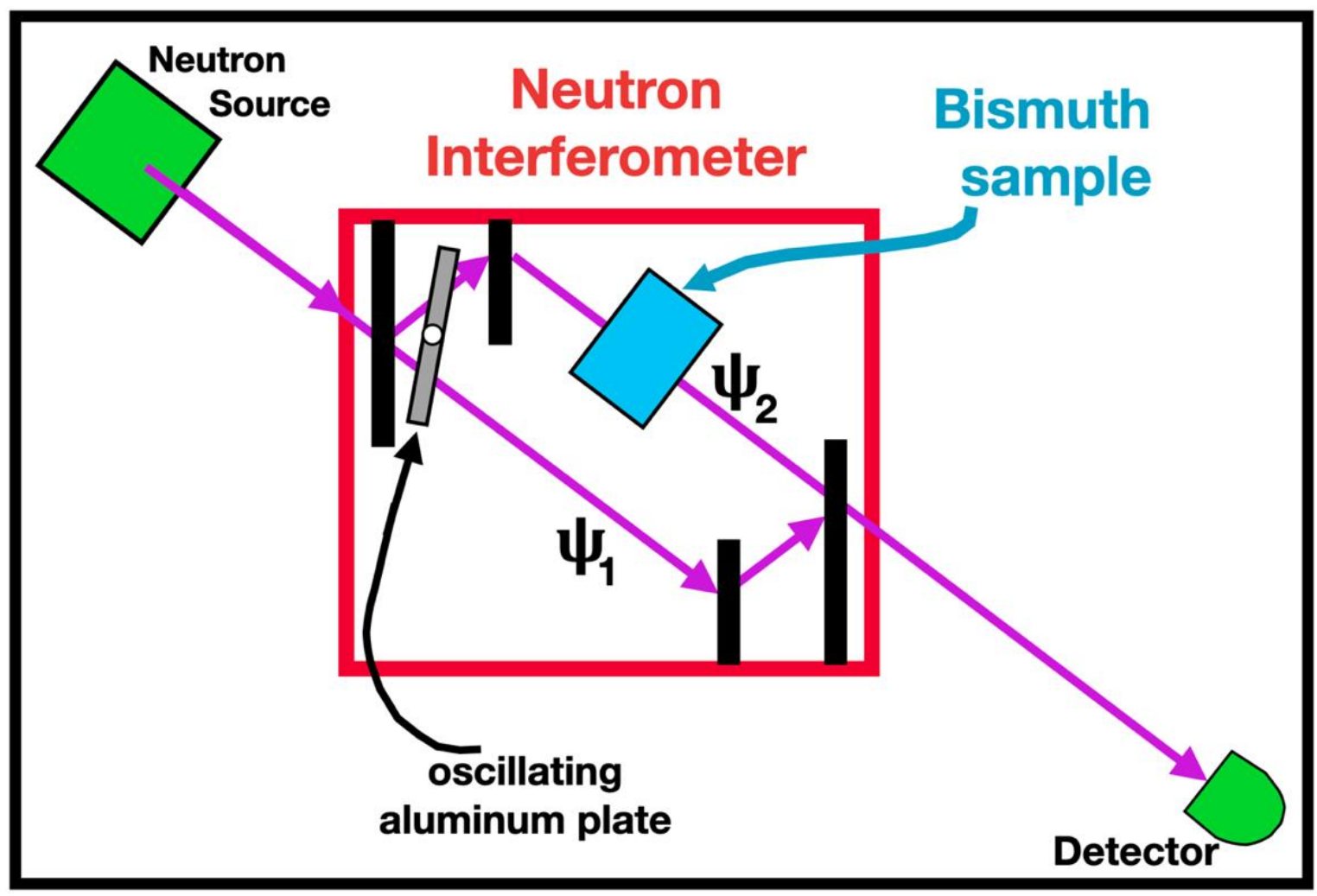

Fig. 19. Kaiser et.al. sent a beam of neutrons into an interferometer, where silicon blades (in black) divided it into $\psi_{1}$ and $\psi_{2}$ which acquired an oscillating phase shift relative to one another. The $\psi_{2}$ beam passed through a bismuth sample that slowed it down. After the beams were recombined, the height of sinusoidal waves diminished as more and more bismuth was added, eventually diminishing to zero interference when the bismuth sample is $12 \mathrm{~mm}$ or more in thickness.

They then repeated the same experiment with one tiny modification. They inserted a nearly perfect (NP) silicon crystal in the exit stream (Fig. 19), downstream from the interferometer, before the neutrons reached the detector. The crystal focused the beam, decreasing the spread of neutron wavelengths and increasing the height of the center of the Gaussian (Fig. 21). Since that crystal was inserted downstream 
from the interference, it was expected to have no impact on the interference upstream inside the interferometer.

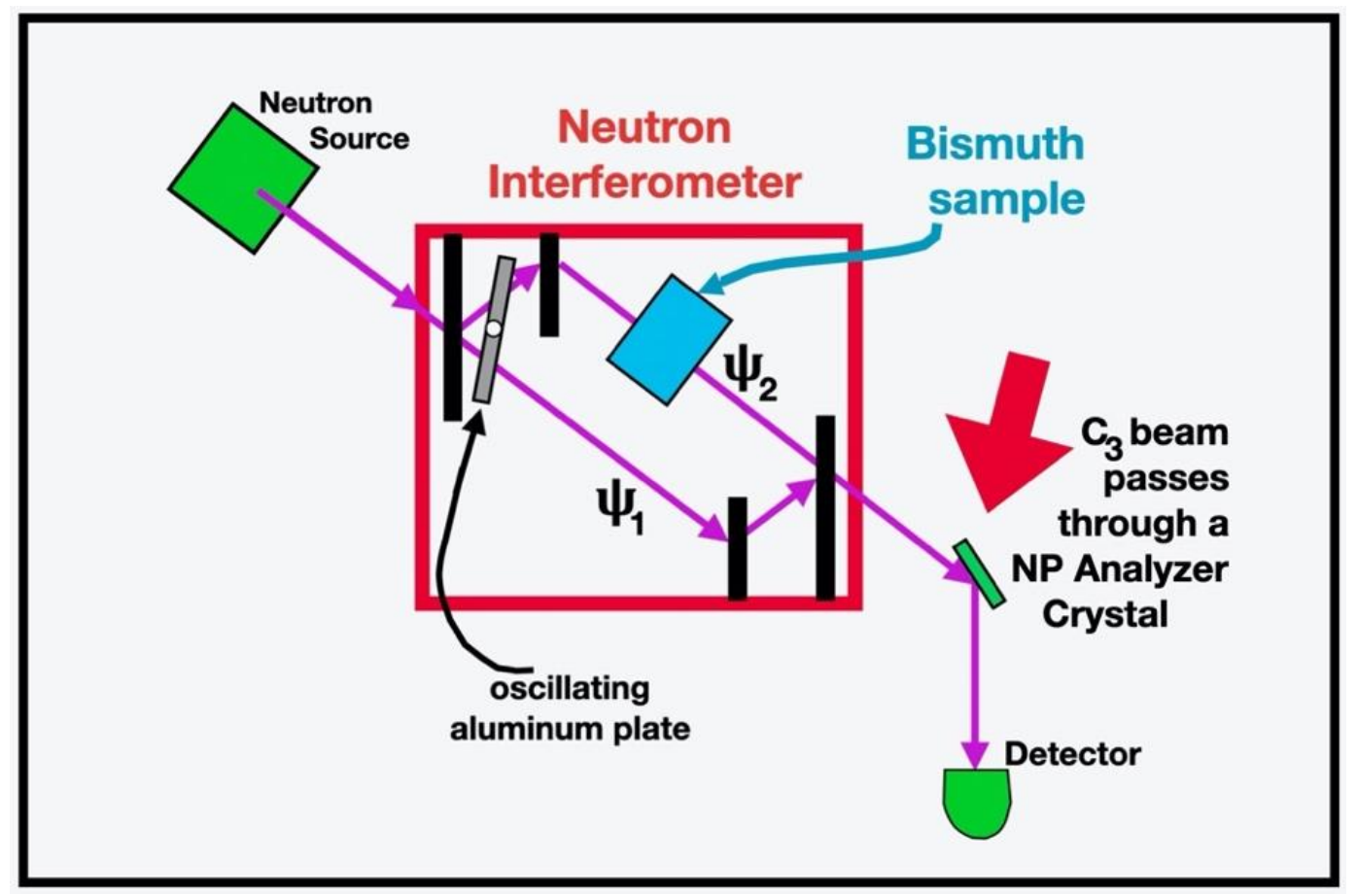

Fig. 20. A nearly perfect (NP) silicon analyzer crystal is inserted in the $C_{3}$ exit beam of the interferometer.

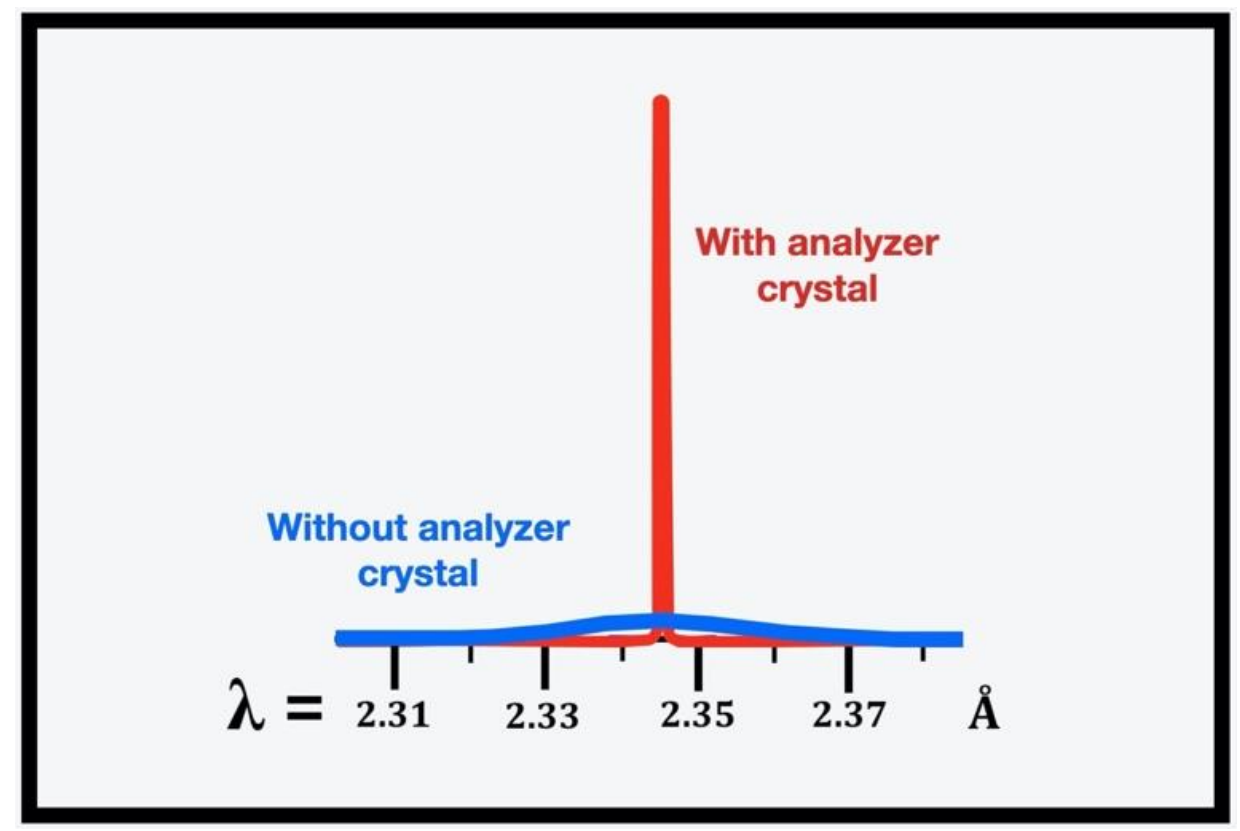

Fig. 21. The nearly perfect (NP) analyzer crystal focused the neutron beam: decreasing the scatter of neutron wavelengths and increasing the height of the Gaussian in the center. It focuses the beam. 
To their astonishment (Table 1 and Fig. 22) the NP analyzer crystal restored robust interference. The researchers said they could not explain these data, and they said that QM could not explain these data.

\begin{tabular}{|c|l|l|}
\hline $\begin{array}{c}\text { Bismuth } \\
\text { width } \\
\text { in } \mathbf{~ m m}\end{array}$ & $\begin{array}{l}\text { Amount of interference in the } \mathrm{C}_{3} \\
\text { Direct Beam } \\
\text { Without Analyzer Crystal }\end{array}$ & $\begin{array}{l}\text { Amount of interference in the } \mathrm{C}_{3} \\
\text { Beam With } \\
\text { NP Analyzer Crystal }\end{array}$ \\
\hline $0 \quad \mathrm{~mm}$ & $100 \%$ & $100 \%$ \\
\hline $4.01 \mathrm{~mm}$ & $57.3 \pm 1.0$ & $97.1 \pm 5.1$ \\
\hline $12.26 \mathrm{~mm}$ & $8.0 \pm 0.8$ & $89.6 \pm 4.4$ \\
\hline $16.15 \mathrm{~mm}$ & $1.8 \pm 0.8$ & $86.0 \pm 4.8$ \\
\hline $20.08 \mathrm{~mm}$ & $2.9 \pm 0.6$ & $95.2 \pm 5.2$ \\
\hline
\end{tabular}

Table 1. Height of the sinusoidal curve. (Data are copied from the righthand column of Tables III and VI, of the Kaiser, et. al. 1992 article.)

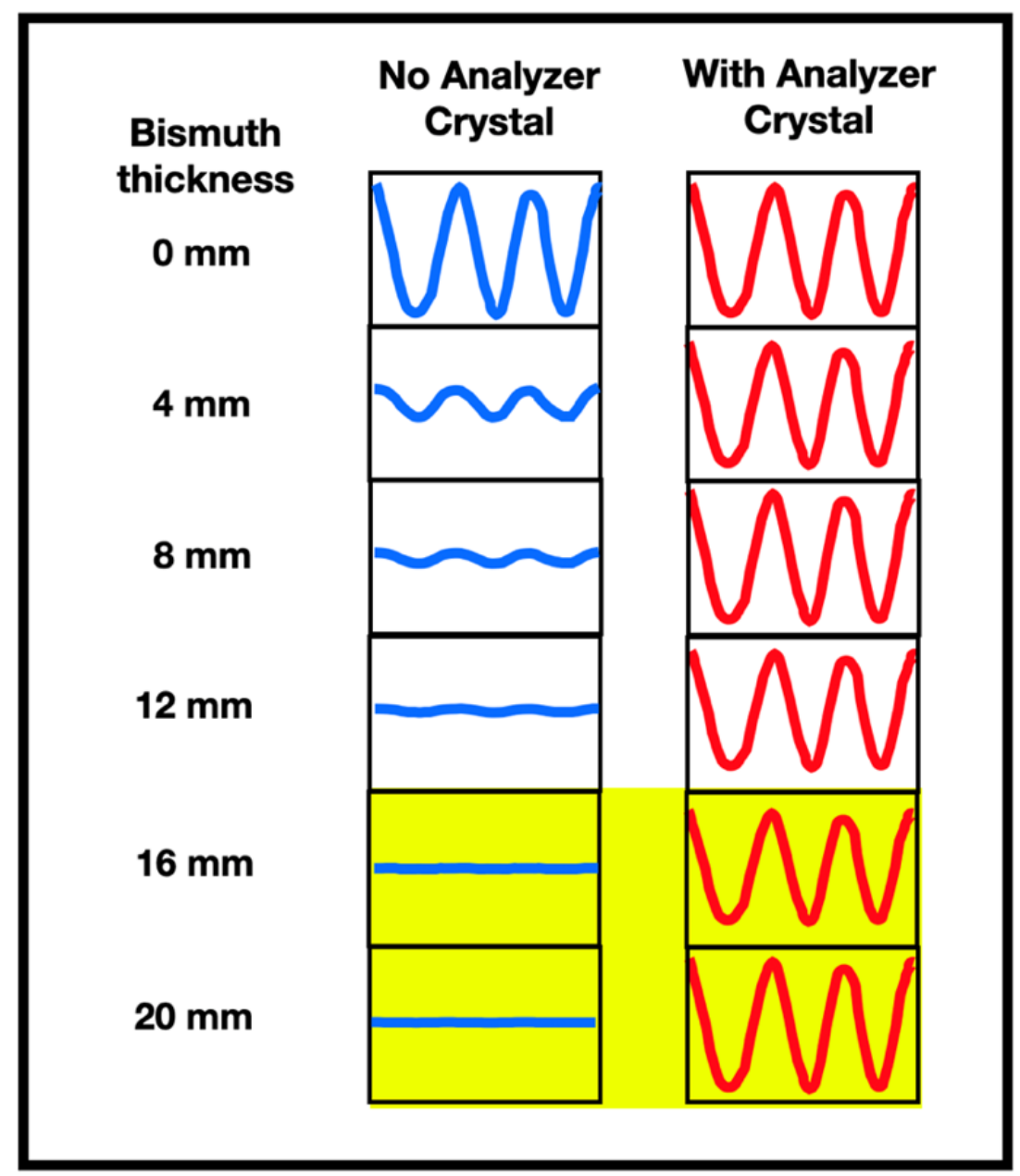

Fig. 22. Comparison of the interferograms with no NP analyzer crystal, compared to the presence of an NP analyzer crystal. In the yellow area, with 16 or $20 \mathrm{~mm}$ of bismuth, all interference died out (center yellow area) until an analyzer crystal was inserted, whereupon robust interference was restored (lower 
right yellow area). These curves come from the top and bottom graphs of Fig. 9, page 40 of the Kaiser, et. al. 1992 article.

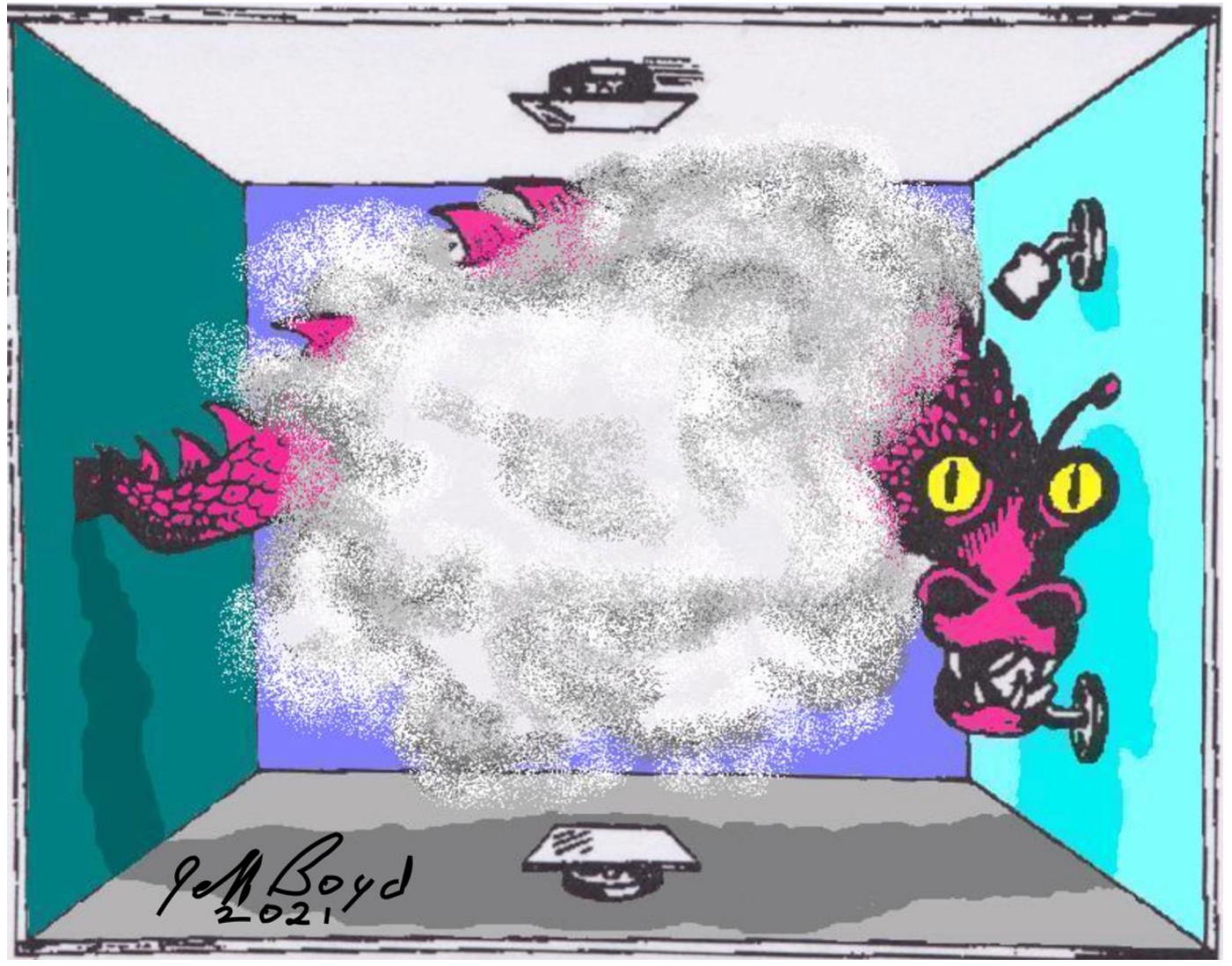

Fig. 23. Kaiser and his team could not explain their data and cited "Wheeler's smoky dragon" as the best explanation they had. It is unusual to find a cartoon offered as a scientific explanation in Physical Review $A$. The dragon was meant to symbolize those unexplainable things that suddenly emerge from the fog in quantum experiments. This cartoon contradicts the idea that "Quantum mechanics can explain all experiments." It suggests that quantum experiments are haunted by a dragon.

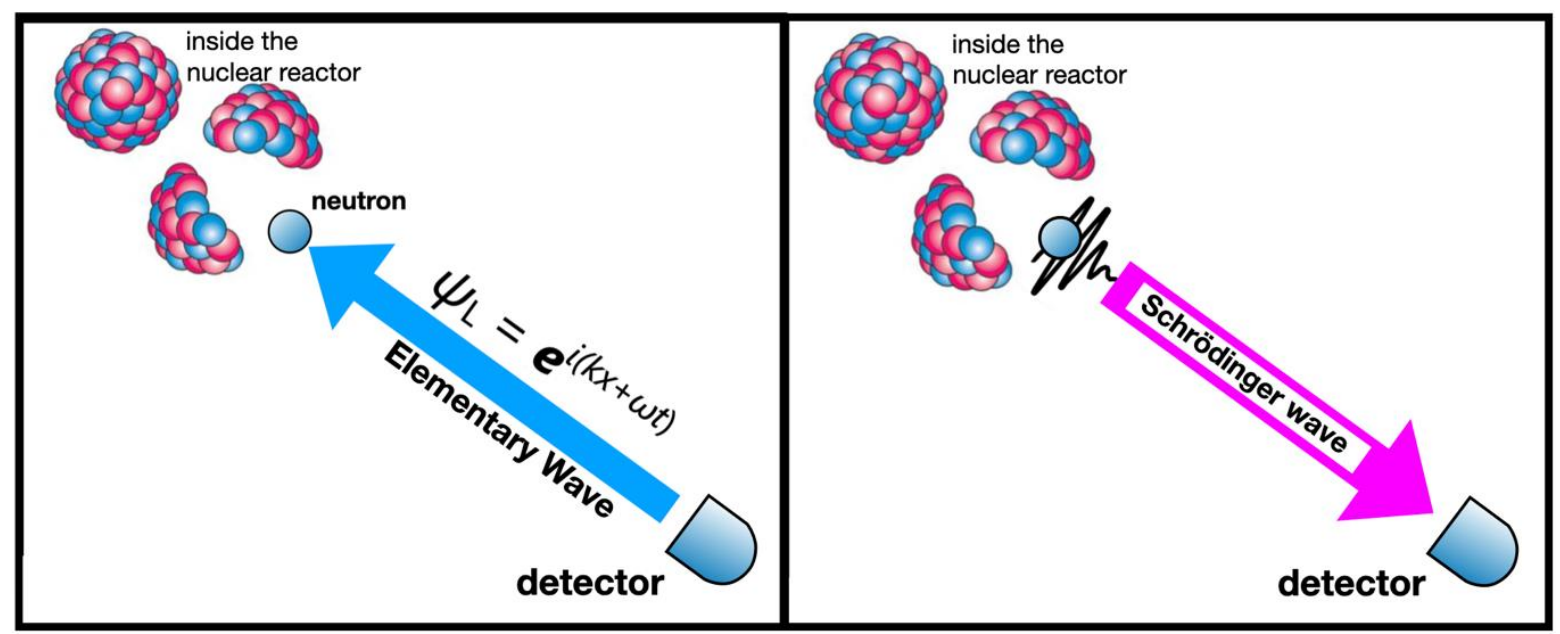

Fig. 24. This is our proposal for how to explain the data: waves travel in both directions.

An elementary wave (in blue) starting at the detector travels backwards through the interferometer and enters the nuclear reactor where it recruits a neutron just released from the splitting of an atomic 
nucleus. As the wave reflects off the neutron it become a Schrödinger wave (pink), carrying the neutron to the detector. The data support the idea that waves are travelling in both directions.

The authors (Kaiser, et.al.) said they could not explain why an NP Analyzer Crystal placed downstream from the interferometer would produce such robust sinusoidal curves in the data. They cited Wheeler's smoky dragon Fig. 23, to declare that unexplainable things happen in QM experiments.

We say that if the presence or absence of an analyzer crystal causes or prevents wave interference, then the analyzer crystal must be upstream from the interference. This means waves are travelling in the opposite direction as the neutrons (Fig. 24).

\subsection{Summary of the neutron interferometer data}

Let's review what we know from the Kaiser neutron interferometer data. There are two columns of data (Table 1 and Fig. 22). The middle column shows that the oscillating aluminum plate affects the neutron wave-packets before the bismuth does, which could only happen if there were Schrödinger waves moving from the neutron source to the detector. The right-hand column of data shows that an analyzer crystal restores robust interference inside the interferometer, which could only happen if there were zero-energy plane waves (elementary waves) moving from the detector up into the neutron source. Therefore, the data demonstrates that waves travel in both directions.

These experimental data show that the plane wave (elementary wave, $\psi_{\mathrm{L}}$ ) and the Schrödinger wave $\left(\psi_{R}\right)$ co-exist, moving in opposite directions (Fig. 24).

In summary, in this and in many other experiments the blue arrow (called an "elementary wave" in Fig. 24) travels from the detector to the particle source, and a quantum particle follows that elementary wave backwards to the detector. The point of origin and the final target are the same: the detector.

\subsection{The double slit experiment explained by TEW}

We now discuss the double slit experiment which is the most famous of all quantum experiments. According to Richard Feynman QM does not know how to explain this experiment. Feynman said the double slit experiment contains what he called the "Central Mystery" of QM. Our model can explain the double slit experiment.

The detector is called the "target screen," which is on the right. And particle $\alpha$ (alpha) is on the left, inside the particle gun. In between them, in the middle, there is a barrier with two slits, as you know. From any point, such as point " $\mathrm{z}$ " on the target screen, Elementary Waves, which are the blue arrows, go forth in these arcs. Then they go backwards through the two slits, A and B. And in proximity to the particle gun the ones through $A$ interfere with the ones through $B$. So, in our model all wave interference is near the particle gun. No wave interference is near the target screen.

So as the waves from point " $\mathrm{z}$ " on the target screen impinge on particle $\alpha$ inside the gun, they interfere with each other, and that interference affects the amplitude with which a given wave from a given point on the target screen impinges on the particle gun.

Depending on the location of " $\mathrm{z}$ " on the target screen the wave passes through slit A before, at the same time as, or after it passes through slit B. The waves through A and B cause wave interference (constructive, destructive, or intermediate) as they impinge on the particle gun. The interference determines the amplitude of the wave as it impinges on particle $\boldsymbol{\alpha}$.

Waves from all other points on the target screen (each of which has a different PDE wave equation) likewise converge on $\boldsymbol{\alpha}$, each with its own amplitude. Based on random chance the particle chooses to respond to only one of the incident waves. "Respond to" means that it becomes reflective instead of transparent (see Fig. 16 for definition of the terms "reflective" and "transparent"). 


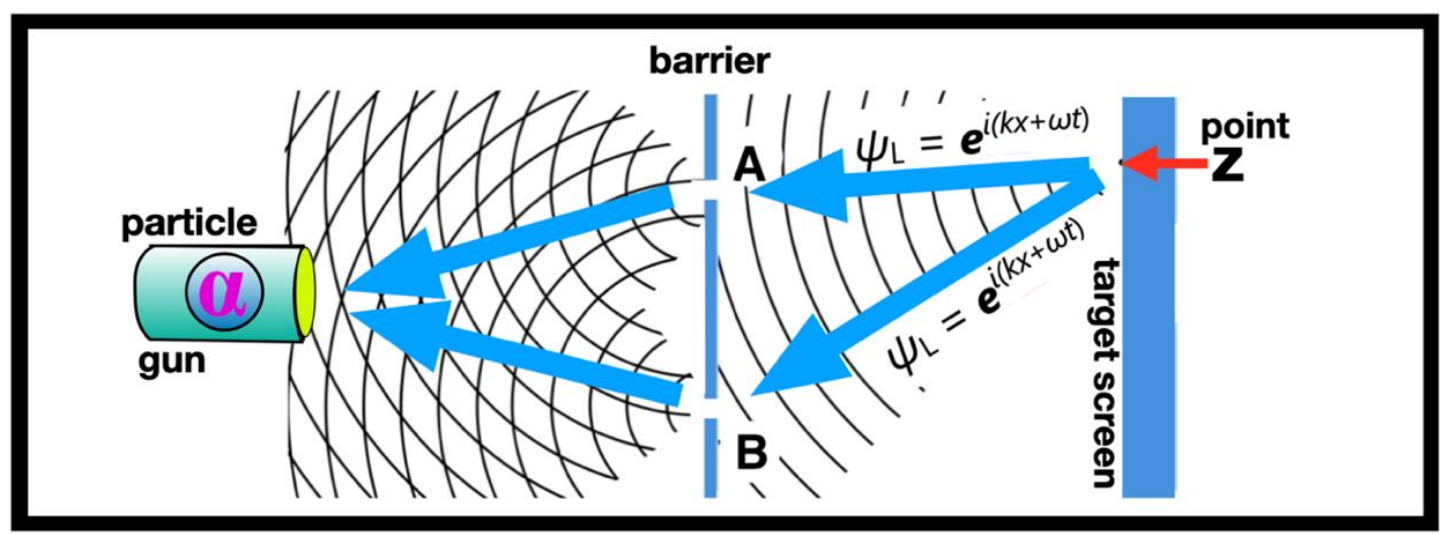

Fig. 24. In a double slit experiment each point " $z$ " on the target screen emits Elementary Waves moving to the left in arcs or semi-circles.

We have only shown waves from one point " $z$ ". But all the different points, a zillion points on the target screen are likewise putting forth Elementary Waves which are arriving at the particle gun with various intensities. The waves from point " $z$ " do not interact with, do not add together with, do not interfere with waves from other points on the target screen.

Each point on the target screen gives rise to a linear partial differential wave equation $\left(\mathbf{u}_{\mathbf{t t}}=\mathbf{c}^{\mathbf{2}} \mathbf{u}_{\mathbf{x x}}\right.$ which yields a solution $\psi_{\mathrm{L}}=\boldsymbol{e}^{i(k x+\omega t)}$ which are linear differential equations. But the linear equation from each point on the target screen is a unique wave equation, different from the other points on the target screen. So, each separate point on the target screen gives rise to a separate linear partial differential equation. This is the key to understanding the double slit experiment. Although two waves (i.e., 2 solutions to the PDE) can be added together to make another solution, IF both waves come from the same PDE (which means they originate at the same point in space), you CANNOT add together two waves from different PDEs (which means they originate at two different points in space). Therefore, although we call these "plane waves", there are no planes crossing the space as a train of vertical lines moving right to left: i.e., Fig. 17 is not how Elementary Waves behave.

Now let's turn our attention to particle $\boldsymbol{\alpha}$ inside the particle gun. Mathematician John von Neumann had asked a question that no one was able to answer. He said, the Schrödinger equation is a deterministic equation. So, how does the randomness get into QM?(64) We can answer that question. Randomness comes from the particle, which we've called alpha $(\boldsymbol{\alpha})$. The particle located in the gun makes a random choice among the incoming Elementary Waves. That choice is influenced by the amplitude with which the waves present themselves. Wave-function collapse occurs when the particle is emitted from the gun in response to, following backwards, one incoming Elementary Wave.

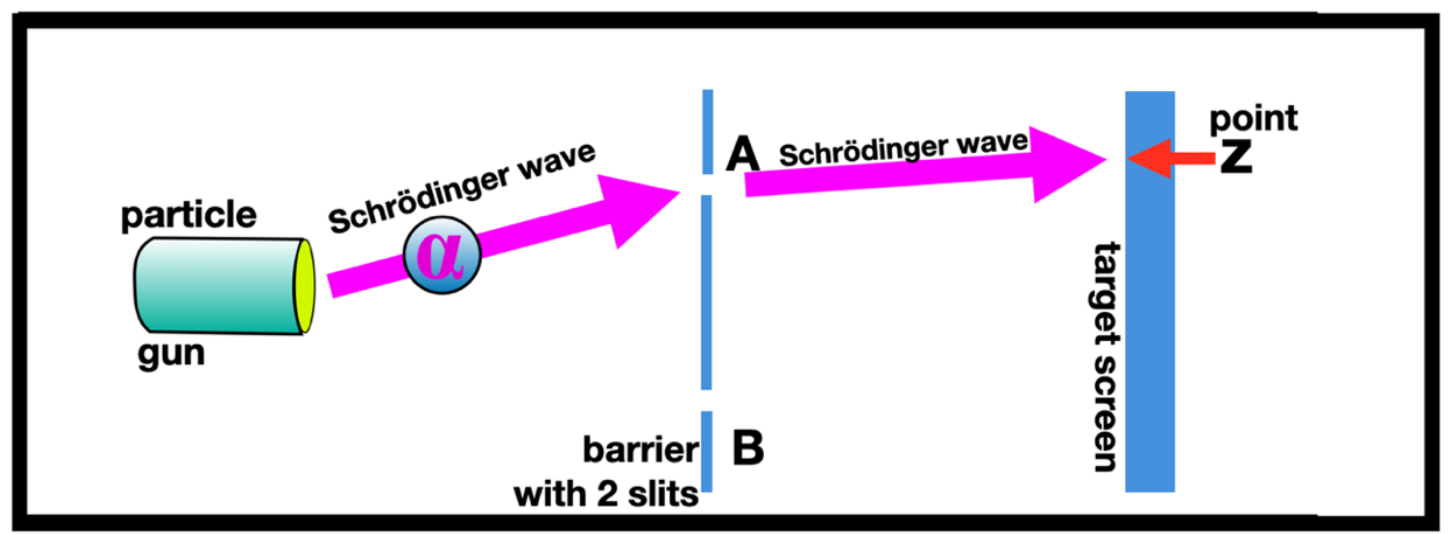


Fig. 26. When particle $\boldsymbol{\alpha}$ chooses the elementary wave from point " $z$ ", that choice constitutes wavefunction collapse, after which it becomes a deterministic experiment.

After the gun is fired, particle $\boldsymbol{\alpha}$ is immune to any wave interference. It then follows its chosen wave $\psi_{\mathrm{R}}$ (which is now a Schrödinger wave) through one and only one of the two slits (it doesn't matter which slit). It follows $\psi_{R}$ with a probability of one, and inevitably makes a dot at precisely that point " $z$ " from which its elementary wave originated.

We know from Brownian motion that particles are inherently erratic, so it is easy to believe that randomness in QM comes from particles, not from the Schrödinger equation.

Notice that after the particle leaves the gun (Fig. 26), the particle follows its trajectory with a probability of one. The trajectory is exactly the pathway used by the incoming Elementary Wave from point " $z$ " to the gun. The particle goes through only one of the two slits. It doesn't matter which slit. The particle inevitably makes a dot at point " $z$ " from which its specific Elementary Wave originated.

Thus the double slit experiment is a random and probabilistic experiment until the particle is emitted from the gun. After the particle leaves the gun the experiment is deterministic. When particle $\boldsymbol{\alpha}$ selects the incident wave from point " $z$ ", that point becomes its destiny. Point " $z$ " pulls the particle home, as if there were an elastic band connecting particle $\boldsymbol{\alpha}$ and point " $z$."

A moment ago, we said that waves from point " $z$ " don't add together with waves from other points on the target screen. The reason that is important is because the wave from point " $z$ " if it happens to be the one that triggers a response from particle $\alpha$, then $\alpha$ will make a dot at point " $z$ " and not at some other point.

We have shown previously that this mechanism will produce precisely the wave interference fringe pattern we expect on the target screen. (4) What is that image on the target screen a picture of? It is a picture of the elementary waves impinging on the particle gun. Remember this remarkable fact: you have seen an actual picture of the elementary waves!

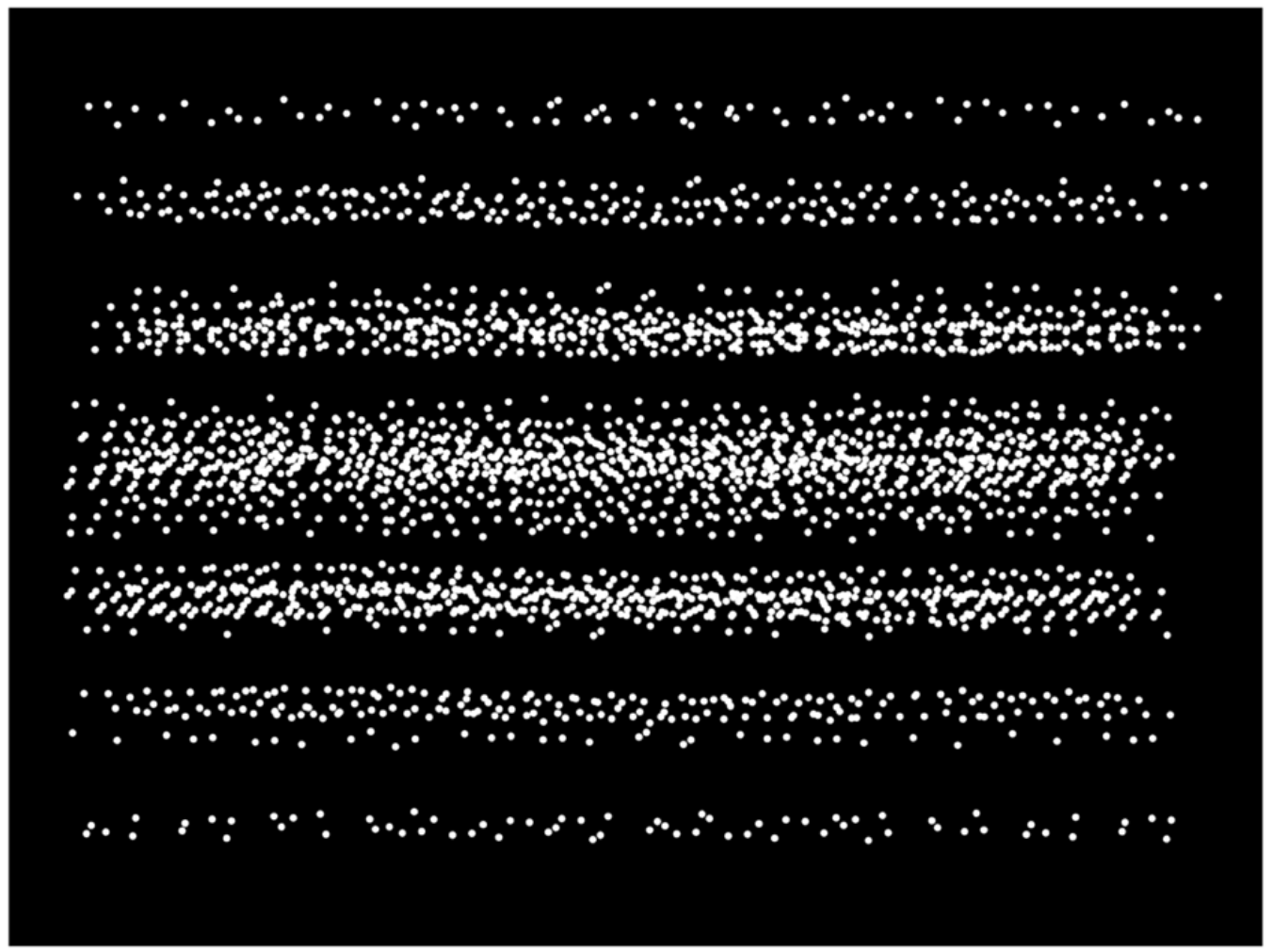

Fig. 27. This is a picture of the zero-energy elementary waves impinging on the particle gun.

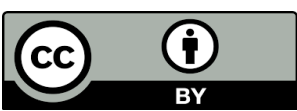




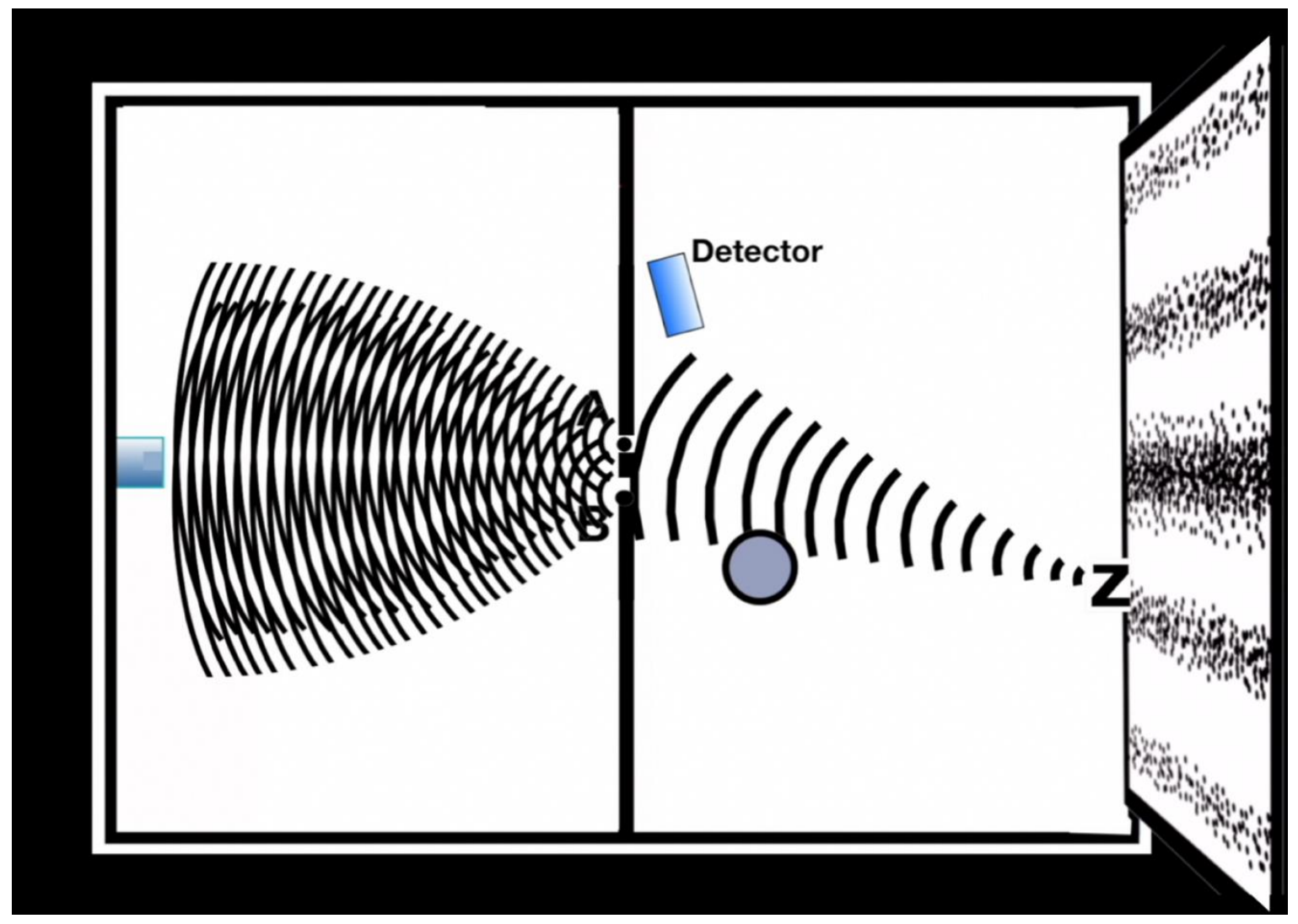

Fig. 28. This shows how the Elementary Waves impinging on the particle gun (left) are displayed as an interference fringe pattern on the target screen (right side). This only happens if the little lamp is off inside the apparatus (grey circle).

\subsection{Complementarity}

"Complementarity" in a double slit experiment means that if we know which slit a particle used then the interference wave pattern on the target screen vanishes. This has caused endless speculation about how human consciousness affects quantum experiments. We reject that speculation: it violates common sense.

TEW says that complementarity is caused by the equipment. In order to know which slit a particle uses, we must introduce a low-energy lamp into the equipment. That small amount of energy is infinitely more than the zero-energy of the elementary waves, and the lamp poisons those waves. The "poisoning" means that each wave ceases to remember its place of origin (Fig. 29).

As you recall, elementary waves from a point like " $z$ " have a linear partial differential equation describing their wave. Any two solutions to such an equation can be added together linearly, and the result is still a solution. With no lamp, the waves from point " $z$ " move to the left in an arc, and then when they pass through the two slits $(A \& B)$ there is wave interference impinging on the particle gun.

However, elementary waves from different points of origin, like $z_{1}, z_{2}, z_{3}, \ldots z_{n}$, each have a different PDE wave equation and therefore waves originating from different points cannot be added together. The poisonous effect of the lamp and detector is to cause the elementary waves to act as if they have different points of origin. Thus, the waves from point " $z$ " through slit $A$ act as if they originated at slit $A$, and therefore have a different partial differential equation than the waves passing through slit $B$. Much has been written about complementarity, meaning that we see a wave pattern on the target screen only if we do not know which slit a particle used. We claim that complementarity is caused by the equipment, and not caused by human consciousness.

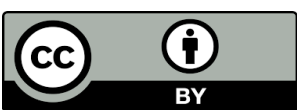


In order to know which slit is used, we must introduce a tiny lamp and detector inside the experiment. Let's say that it's a lamp that emits an infinitesimal amount of energy. The infinitesimal amount of energy from the lamp poisons the Elementary Waves which carry zero-energy, poisons those waves as they pass backwards through the two slits, so they no longer cause wave interference. By this we mean that the waves forget that they were born at point " $z$ ". The wave coming backwards through slit A thinks it was born at slit $A$. The one through slit $B$ thinks it was born at slit $B$. Therefore, there is no wave interference, for the obvious reasons.

What are the "obvious reasons"? Each point from which a wave emanates gives rise to a separate partial differential wave equation (sorry about that math there), therefore you cannot add together waves from different points of origin. The wave coming backwards through slit $A$ thinks it was born at slit $A$, and no longer acts like a sibling of the wave that thinks it was born at slit B. By "non-sibling" we mean the waves will not allow you to add them together. Two Elementary Waves can be added together if and only if they share the same linear equation, which only happens if they have the same point of origin.

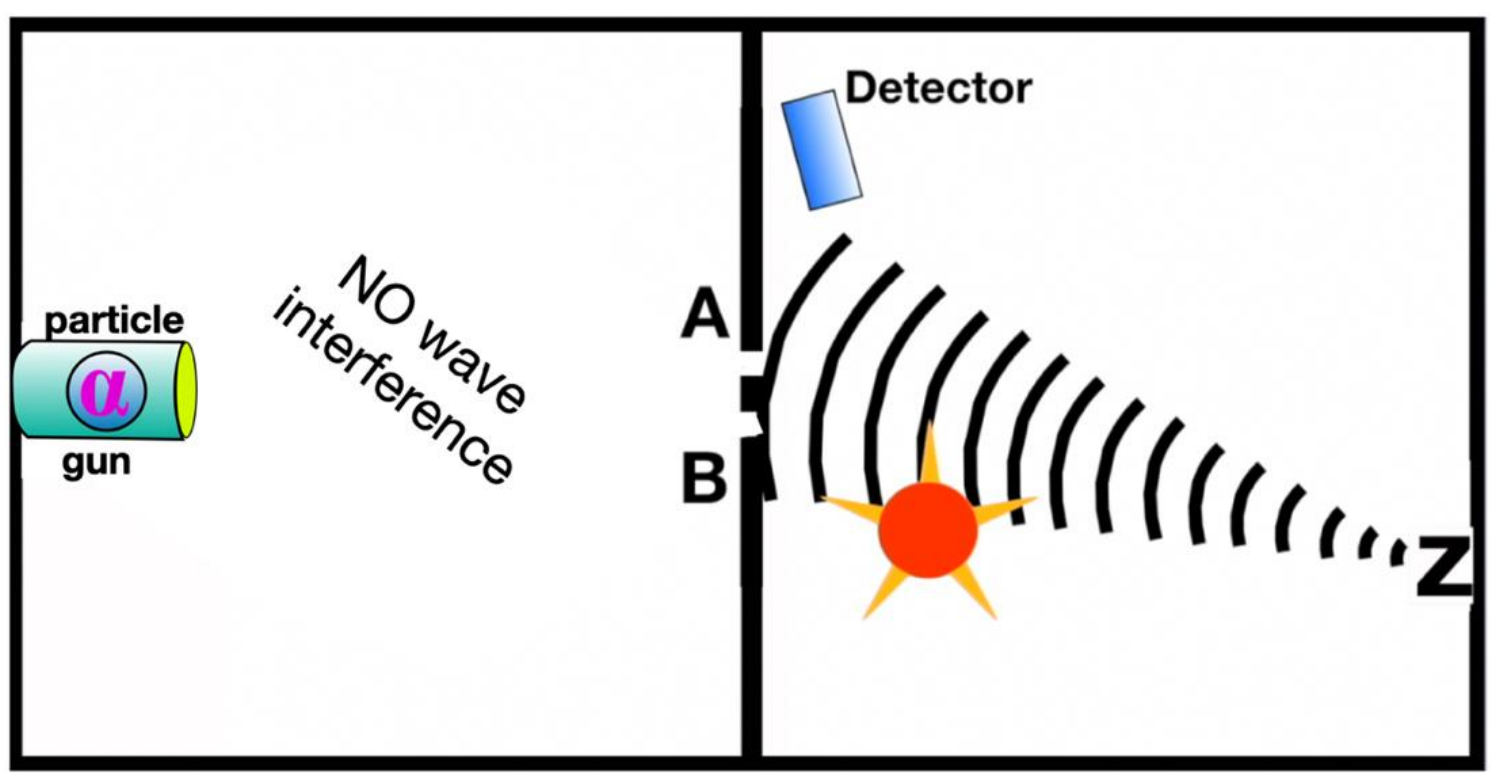

Fig. 28. To discover which slit a particle uses in the double slit experiment, we insert a lamp and detector inside the apparatus.

The energy of the lamp is infinitely more than the zero-energy of the elementary waves coming from point " $z$ " and that causes the elementary waves to lose their memory of where they came from. After passing through the two slits, the waves through slit " $A$ " believe they originated at slit " $A$ ", and the waves through slit " $B$ " believe they originated at slit " $B$ ". Therefore, those waves now have different partial wave equations and cannot be added together linearly.

What is the meaning of the wave pattern on a target screen (Fig. 28)? That wave pattern is a picture of the wave interference of Elementary Waves impinging on the particle gun. But we just said that if there's a lamp in the experiment there is no wave interference, and therefore the final pattern on the target screen will show, "No wave interference!" As we turn the little lamp off and on and off and on, notice that the wave interference impinging on the particle gun appears and disappears, and so does the wave pattern on the target screen. This explains complementarity.

As a result, there is no wave interference as the elementary waves impinge on the particle gun. Since the pattern on the target screen of a double slit experiment is a snapshot of wave interference in proximity to the gun, and since there is no such wave interference when the little lamp is "ON," therefore the target screen says, "No wave interference." 
This explains "Complementarity." The core issue is shown in Fig. 17, which shows that different points of origin cannot produce waves consisting of parallel planes marching across space, because each point generates its own unique partial differential equation.

\subsection{A proposed experiment}

In almost all experiments QM and TEW predict identical outcomes. To distinguish them we need a moving part. We therefore insert a powerful laser that can close one of the two slits of a double slit experiment, as shown in Fig. 30.

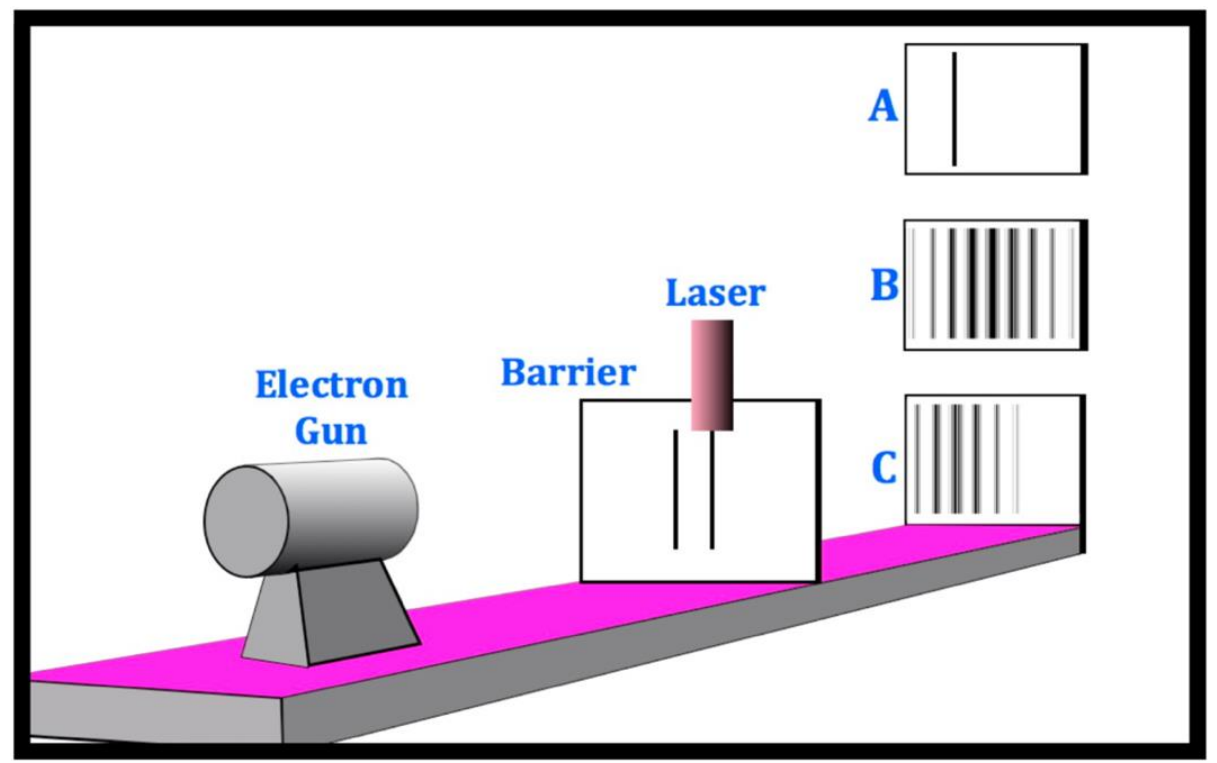

Fig. 30. In this proposed variation on a double slit experiment particles are fired one at a

time,followed by a pause. The powerful laser above the right-hand slit is "OFF" until a particle is fired, whereupon the laser instantaneously closes the right-hand slit. There are three possible outcomes, as shown on the right. A. is what QM predicts as final data because this is a single lit experiment. B. is what would happen if the laser were always "OFF". C. is the final data that we would observe if TEW were correct.

QM and TEW differ with respect to the timing of wave interference in a double slit experiment. TEW says all wave interference occurs before a particle is fired from the gun. QM says all wave interference occurs after the firing of the particle gun. Therefore, if we use a laser to close one of the slits at the same instant when the gun is fired, then we can isolate the timing of the wave interference and discover which theory is correct.

No one has ever constructed nor conducted this experiment. The reader is encouraged to do so.

\subsection{Schrödinger's cat}

The Schrödinger's cat paradox concerns the timing of wave-function collapse. It is usually said that the phrase "wave-function collapse" means that an observable (a linear operator on a Hilbert space) can take an infinite number of values until a measurement is made, but then the wave-function collapses, and the observable takes only that one value that was measured. If it is measured a second time, it still takes that value which was previously measured.

We translate the phrase "wave-function collapse" from mathematical to ordinary language, and from Hilbert to Euclidean space. The phrase means that many things are possible and if only one of them can become reality, then when we choose which one, that choice is permanent. For example, you may flirt with and date many attractive people. But then you marry one. After that marriage, things are different. 
You cannot go back and play the field as if the marriage had not happened. Marriage is the equivalent of wave-function collapse.

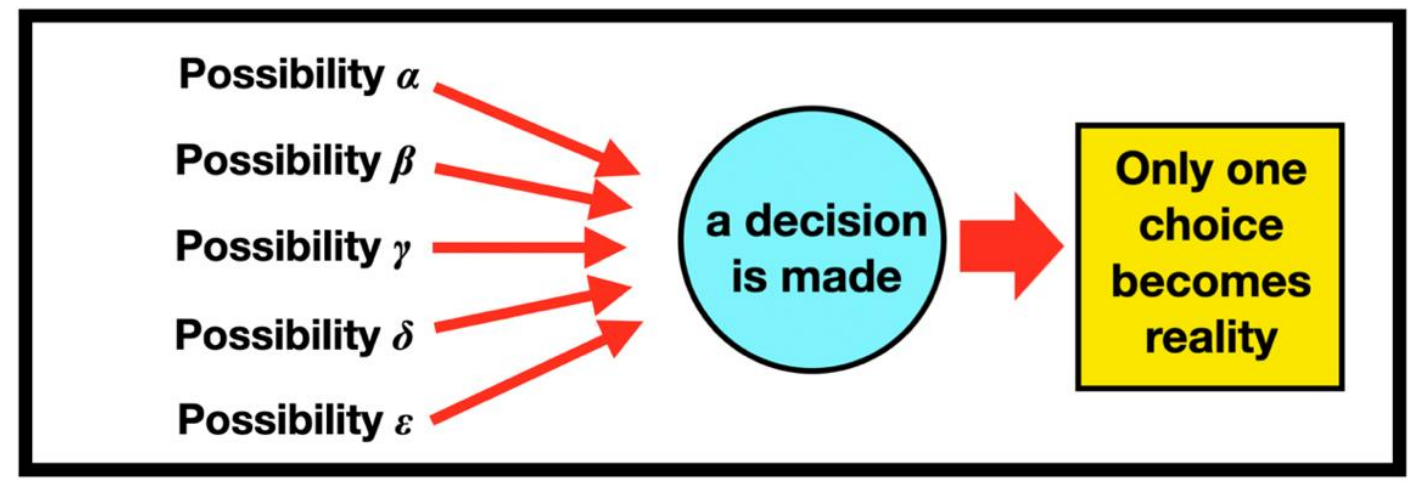

Fig. 31. This is our translation of the phrase "wave-function collapse."

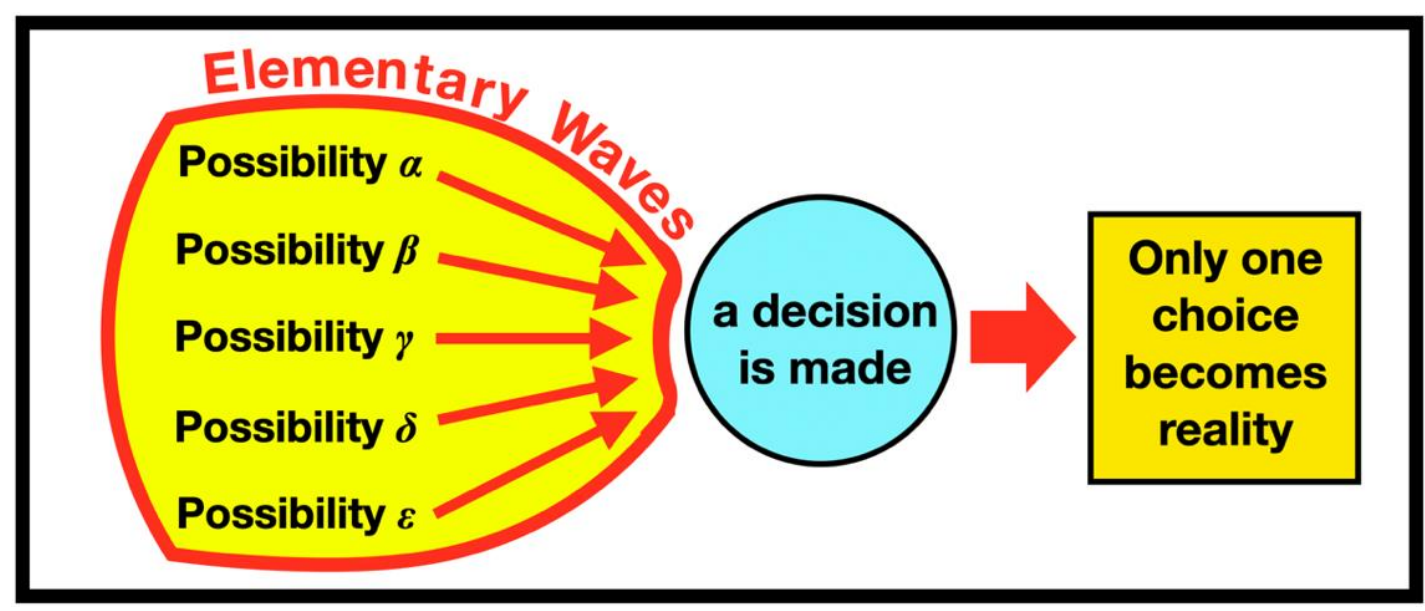

Fig. 32. "Wave-function collapse" means that one elementary wave out of many is selected.

In TEW wave-function collapse often happens at the particle gun when a particle is emitted. After that the final measurement is already determined, even though that measurement has not yet been made.

In TEW wave-function collapse always precedes measurement or observation. This means that TEW avoids the philosophical trap of positivism that QM is stuck with.

The reason for the Schrödinger's cat paradox is that QM says that wave-function collapse only occurs when an observation or measurement is made. Thus, the cat, dead or alive, is required to be in a superposition of both states until the lid of the box is opened, and the cat is observed, whereupon wavefunction collapse occurs, and the superposition is decided.

In TEW the decision (death vs. life) is decided before an observation is made. It happens when the hammer randomly falls, or does not fall, smashing a vial of cyanide causing feline brain death.

There is still a superposition in TEW. It is a superposition prior to the hammer-fall, a superposition of two future states that is undecided until the hammer falls. The superposition is this: "The cat could soon be dead or alive, so the cat's future is dangling by a thread."

This author has experienced such a superposition of states. Once he was driving his car at highway speeds when he was exhausted and drowsy. If he had fallen asleep at the wheel he might have died or suffered brain damage from a car crash. The crash might have killed someone else. Since that superposition of states had not yet happened, he was anxious, and the anxiety kept him awake until he could stop and find a cup of coffee. 


\subsection{The Purcell effect}

The reason that we have quantum weirdness turns out to be because scientists have only been looking at half of reality, half of nature. They've only been looking at the half of nature that contains energy and matter. It turns out there's another half, equally large, which contains neither energy nor matter. It contains zero-energy Elementary Waves, which, despite having zero-energy, seem to be in control of everything else, giving it shape and direction. Particles then randomly choose to respond to one specific incident Elementary Wave, ignoring all the other Elementary Waves.

You may ask, "How can a zero-energy Elementary Wave accomplish anything?" Consider the Purcell effect to demonstrate that zero-energy Elementary Waves can be in control, even though they have zero-energy. You can take a Rydberg atom such as sodium, cesium, beryllium, magnesium, or calcium and heat it in an oven, and then use a laser to excite the outer electron to a higher energy state and then inject the atom into a micro-cavity. The outer electron will drop to a lower energy level and emit a photon $\gamma$ (gamma) 500 times faster if the "available states," or "mode of the cavity" are "resonant" than if they're not. What does it mean to be "resonant"? It means that the diameter of the cavity is a multiple of $\lambda / 2$ where $\lambda$ is the wavelength of the photon that would be emitted. $(36,50)$

So how does a hot and excited Rydberg atom wandering naïvely into a cavity know the width of the cavity? Does a quantum wave from inside the atom go out and measure the environment, and, if it is inhospitable go back into the atom and tell the electron, "Forget it! Stay where you are"? Of course not.

Rather the "available state" is something with zero-energy that already lives inside the cavity before any excited Rydberg atom arrives. We are going to rename the "available state" and call it an "Elementary Wave." An "available state" is a zero-energy Elementary Wave that lives inside that cavity. So, what these waves do is trigger the electron to use the energy stored up inside the electron.

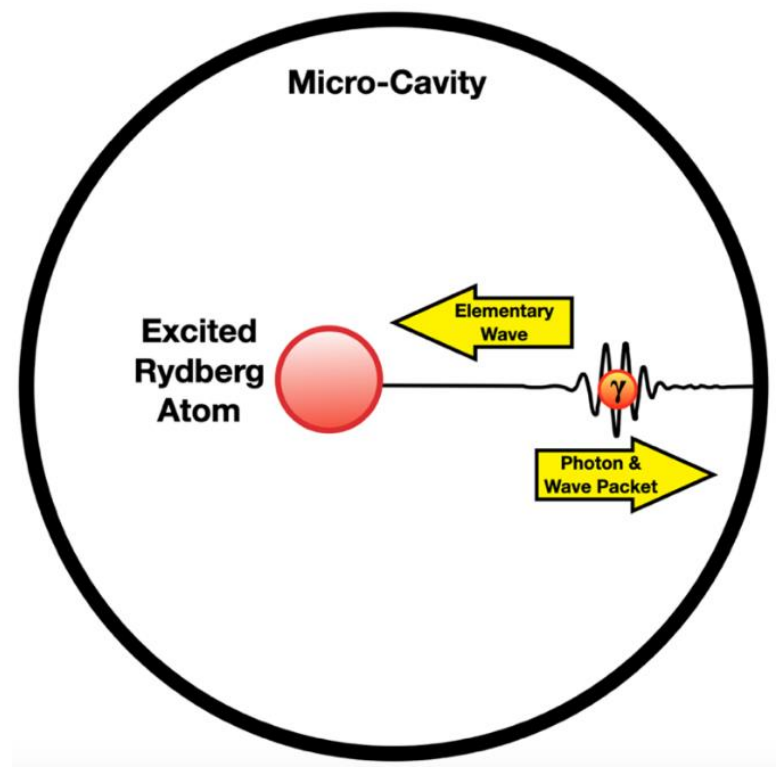

Fig. 33. The Purcell effect. How does the excited electron know the diameter of the cavity, to determine

whether it has a diameter that is a multiple of $\lambda / 2$ ? An "available state" (also known as a "mode of the cavity") resides inside the microcavity. We rename "available state" and call it "Elementary Wave." 
So, the Elementary Wave gives permission or triggers the electron to use its own energy to fall to a lower energy state and emit a photon. That shows how a zero-energy Elementary Wave can be in control even though it has no energy.

When something happens in the quantum world, it is because the quantum phenomenon is seeking to occupy an "available state." Our detector creates an "available state." When we are looking for and expecting to see something, that allows nature to reveal herself to us. It is not that our looking causes things to happen, but our looking allows things to happen, that would not otherwise happen.

Physicists have always said that the available states carry zero energy. Thus, physicists have known since 1946 that something of zero energy can control an energetic process.

\subsection{The TEW explanation of a Bell test experiment}

We said before that we would give you the TEW explanation of the Bell test experiments, which, as you are about to discover, is incompatible with Einstein's local realism, but also differs from the QM explanation, and we think is more down-to-earth and believable than the idea that John Bell had.

Our model (Fig. 14) has a zero-energy blue Elementary Wave going out from the human eye. This implies that everywhere in space there are an infinite number of Elementary Waves going in all directions and at all wavelengths. And if you accept that, which is somewhat hard to believe, but we believe it is true, then that implies that every Elementary Ray, like the blue arrow, has a mate, namely an identical Elementary Ray travelling coaxially in diametrically the opposite direction. We call this a "Bi-Ray", namely an Elementary Ray going in one direction, and an identical one travelling coaxially in the opposite direction. You might ask, "What makes such counter-vailing rays coherent?" What makes them coherent is the photons or particles which are following the Bi-Rays.

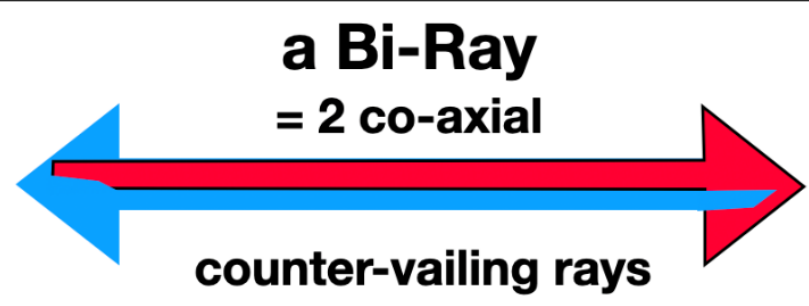

Fig. 34. If nature is full of Elementary Rays travelling in all directions and at all wavelengths, then each ray has a mate, namely an identical ray travelling co-axially in the opposite direction. What makes them coherent is the particles (such as photons) following both (like following a railroad track).

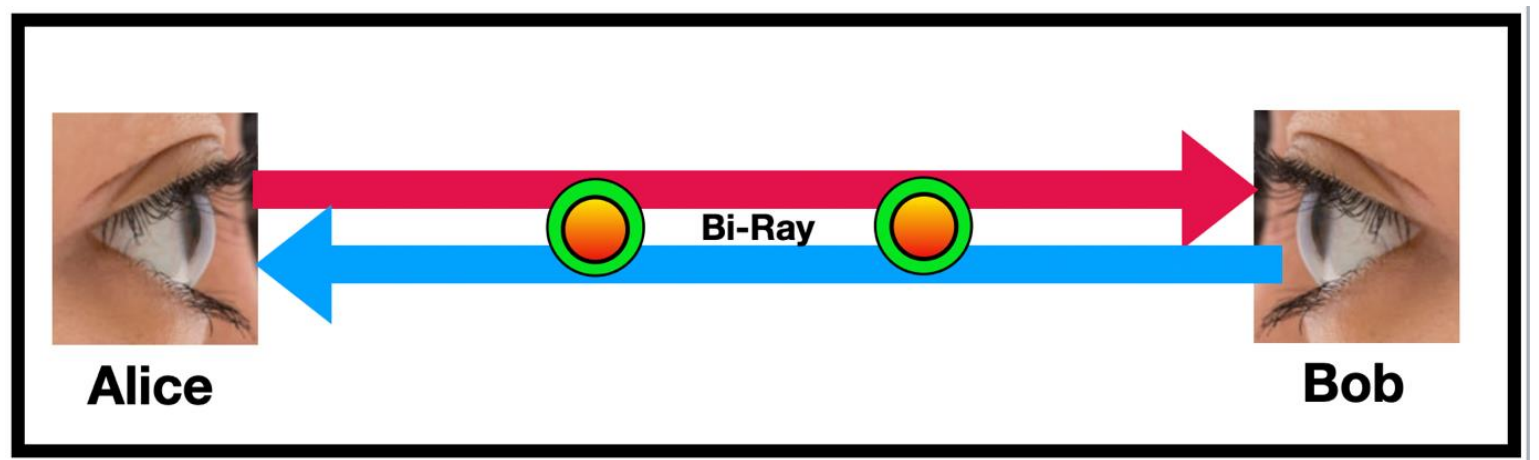

Fig. 35. The Bell text experiments involve a pair of entangled photons originating in the center and moving respectively toward Alice and Bob, both of whom use a polarizer with a random angle to watch for a photon.

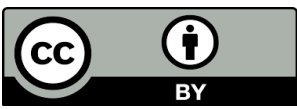


It is useful to think of these Bi-Rays as being like a train track with two rails. A photon, or an entangled pair of photons can travel on that railroad track. It is the particle(s) and not the railroad track which provides the energy.

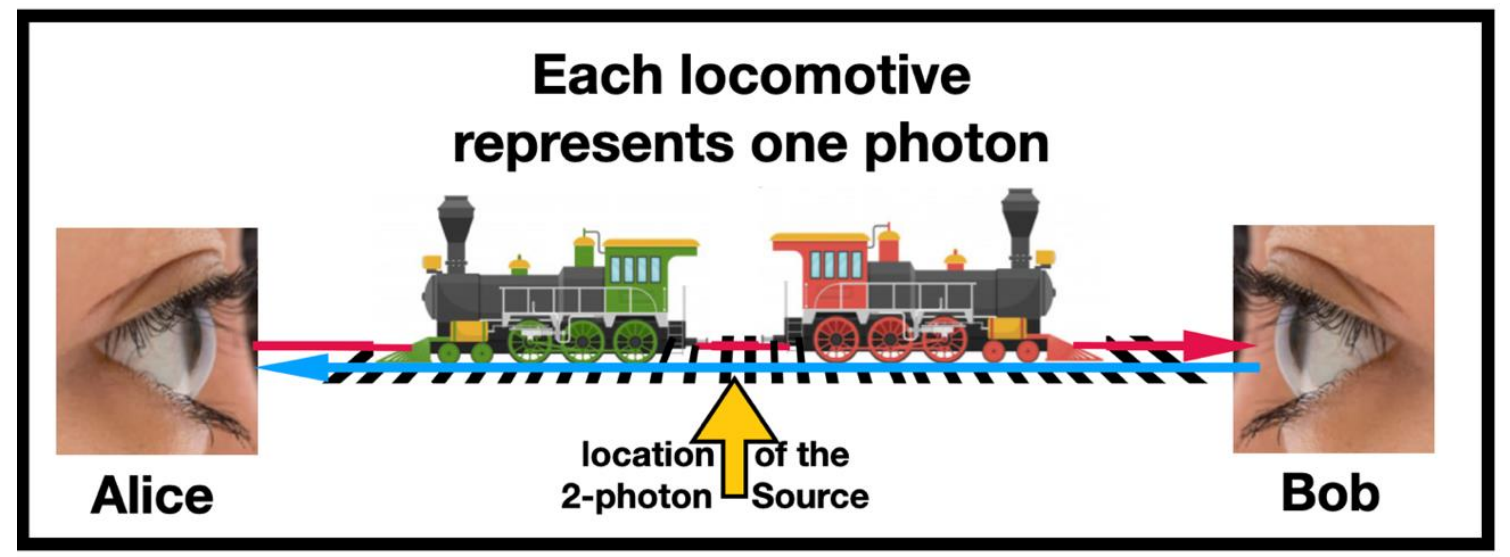

Fig. 36. The Bi-Rays are like railroad tracks which each of the entangled photon follows.

You will notice that each locomotive or photon travels on both rails of the train track. We need to make only one assumption to explain the Bell test experiment results. The assumption is that the probability of a locomotive or photon following the Bi-Ray railroad track is the amplitude of it following one ray times the amplitude of it following the other ray or rail. As we say, that is the only assumption we need to explain the Bell test experiment results, but we are not going to give you the equations in this video.

Given our single assumption, we can predict that the coincidence rate of the Bell test experiments will be $\boldsymbol{P}=\cos ^{2}\left(\boldsymbol{\varphi}_{2}-\boldsymbol{\varphi}_{1}\right)$ or $\boldsymbol{P}=\sin ^{2}\left(\boldsymbol{\varphi}_{2}-\boldsymbol{\varphi}_{1}\right)$, where "P" means "probability" and $\boldsymbol{\varphi}_{1}$ and $\boldsymbol{\varphi}_{2}$ are the random angles of Alice and Bob's polarizers.

In a Bell test experiment both Alice and Bob are looking for a photon through a polarizer set at random angles that keep changing. If there are pairs of entangled photons emitted in the center of this apparatus, then it is easy to prove that the probability or coincidence rate of both Alice and Bob seeing a photon at the same instant, although they are seeing different photons from one another, the coincidence rate forms a sinusoidal curve identical to the results of the Bell test experiments.

Alice and Bob always violate Bell's inequality, but they also differ from QM especially with respect to speed of light and nonlocality. At no point does TEW violate the speed of light, because our stopwatch, in Elementary Wave theory always starts sooner than the particle was emitted.

The difference between cosine and sine is that it depends on what technology is used to generate two entangled photons. For example, in Alain Aspect's experiment he used a calcium cascade source to generate 2 photons with the same polarization, and therefore the coincidence rate he discovered was $P=\cos ^{2}\left(\varphi_{2}-\varphi_{1}\right)$. If Aspect had used a different source (for example, one involving a Wollaston prism) that generated photons orthogonal to one another at birth, then his coincidence rate would have been $P=\sin ^{2}\left(\varphi_{2}-\varphi_{1}\right)$.

In many experiments coincidence rate $=\sin ^{2} \theta$ is used to test for a violation of Bell's inequality. If we rotate the axes, the coincidence rate of $\cos ^{2} \theta$ also gives us results that also violate Bell's inequality. One experiment found a coincidence rate of $\sin ^{2}(\theta+x)$, where the variable " $x$ " varied depending on the time of the day, as the temperature of the equipment changed. That entire family of sinusoidal squared curves violates Bell's inequality. Alain Aspect et.al. reported a coincidence rate of $\cos ^{2} \theta$ in 1982 (Fig. 37). 


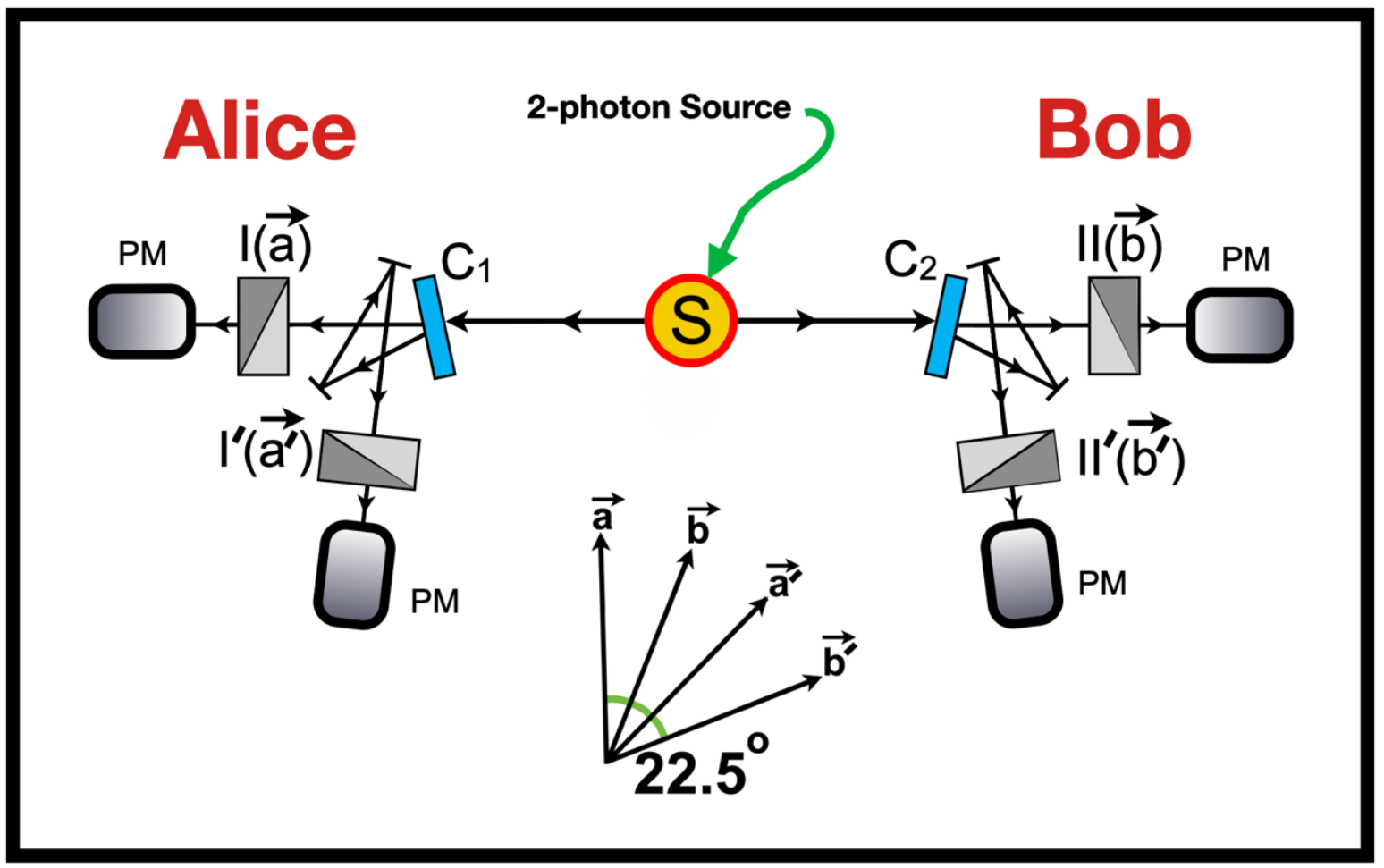

Fig. 37. Equipment used by Aspect, Grangier and Roger in 1982.

In Aspect's 1982 experiment, entangled photons departed the source " $\mathrm{S}$ " and were assigned by a randomizing device ( $C_{1}$ or $C_{2}$ shown in blue) to Wollaston prisms angled at $22.5^{\circ}$ increments, and then observed by photomultipliers (PM). The angles of measurement were chosen to maximize the discrepancies between QM and Einstein's predictions.

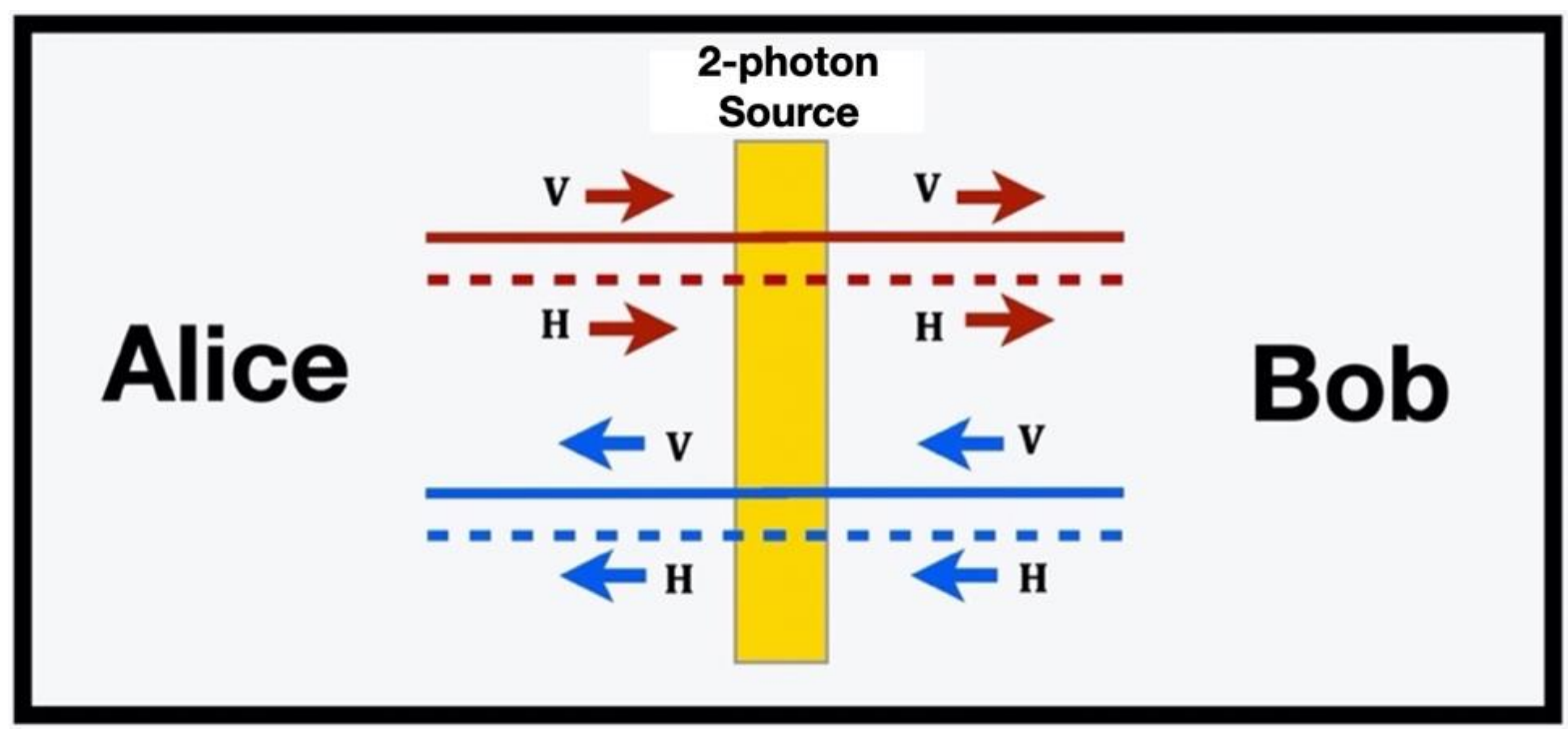

Fig. 38. Using vertical (solid lines) and horizontal (dashed lines) polarized elementary waves, we redraw

Figs. 34-35. We will use red to denote an elementary ray travelling to the right, and blue for one travelling to the left. Note that these polarized waves above apply only to the individual elementary rays. In the Fig. 39 the four Bi-Ray eigenstates are given. 
The angles $\boldsymbol{\Phi}_{1}$ and $\boldsymbol{\Phi}_{2}$ are the random angles of Alice and Bob's polarizers. Neither Alice nor Bob influence or even know what angle of polarization the other person is using. The probability of both Alice and Bob simultaneously seeing a photon (the so-called "coincidence rate") in the $\boldsymbol{\alpha}$ eigenstate is the probability of Alice seeing a photon $\left(\sin \left(\Phi_{1}\right) \sin \left(\Phi_{1}\right)\right)$ times the probability of Bob seeing a photon $\left(\sin \left(\Phi_{2}\right) \sin \left(\Phi_{2}\right)\right)$. This becomes the first line of our equation

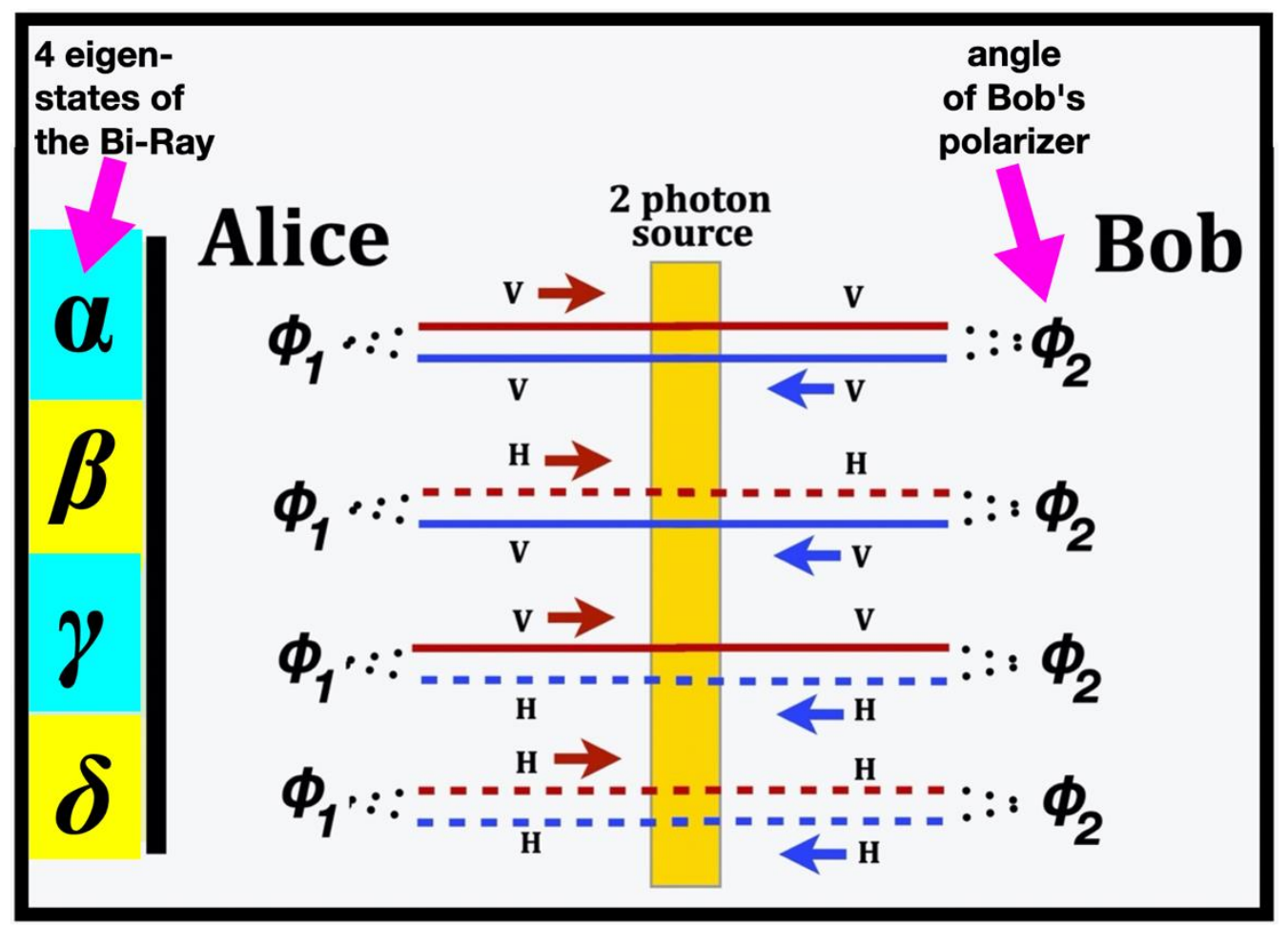

Fig. 39. We define four new eigenstates $(\boldsymbol{\alpha}, \boldsymbol{\beta}, \boldsymbol{\gamma}$ and $\boldsymbol{\delta})$ of the Bi-Ray between Alice $\left(\boldsymbol{\Phi}_{1}\right)$ and Bob $\left(\boldsymbol{\Phi}_{2}\right)$.

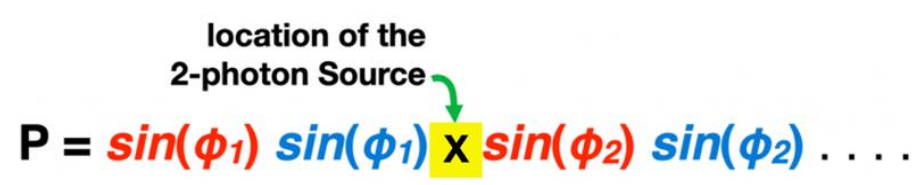




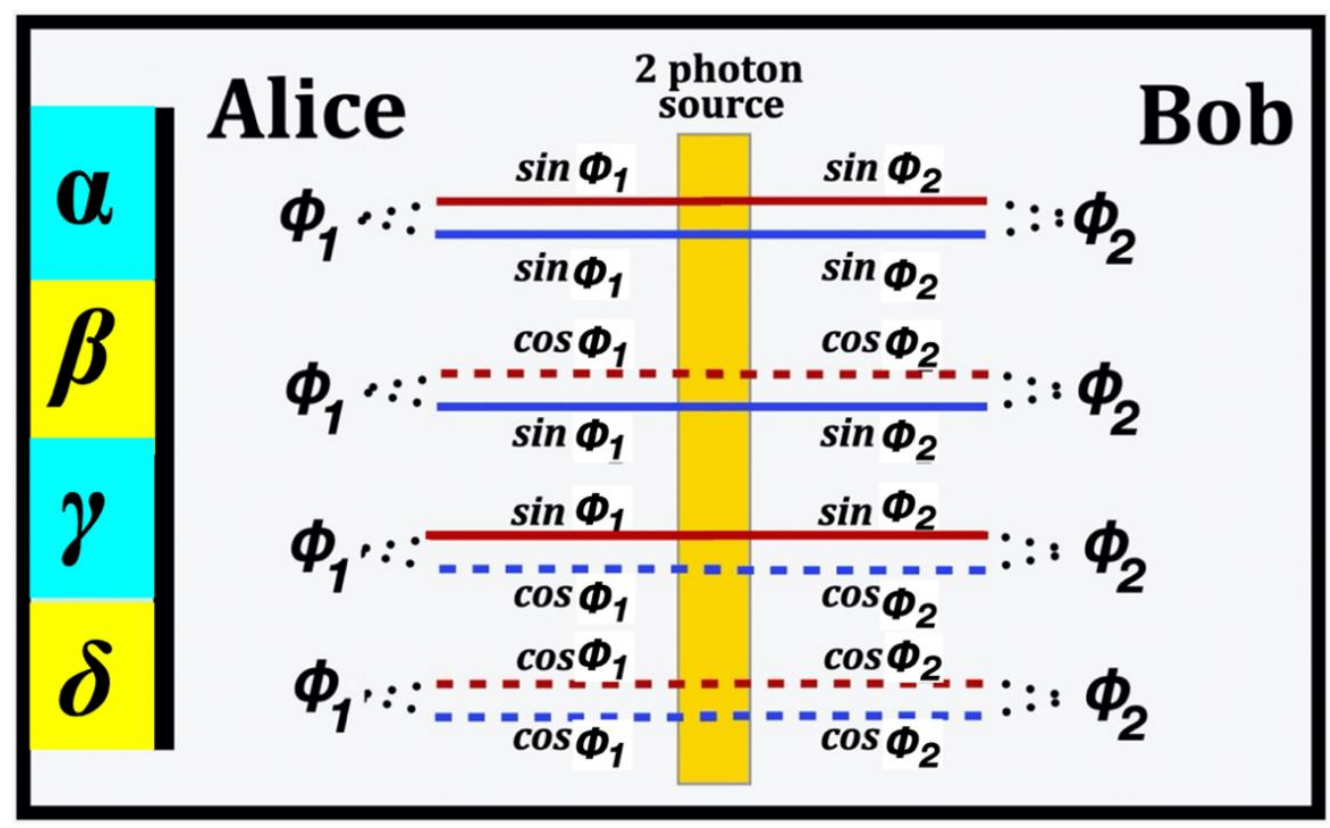

Fig. 40. These sines and cosines show the amplitude for a photon from the source being detected by Alice or Bob in a specific eigenstate when their polarizers have been set to angles $\boldsymbol{\Phi}_{1}$ and $\boldsymbol{\Phi}_{2}$.

Note in Fig. 38-40 that one photon travels to the left and the other photon to the right. Alice's choice of angle $\boldsymbol{\Phi}_{1}$ is independent of Bob's choice of angle $\boldsymbol{\Phi}_{2}$. Alice detects a different photon than Bob.

To find the probability of Alice and Bob simultaneously seeing a photon (called the "coincidence rate"), we add together the four eigenstates $(\boldsymbol{\alpha}, \boldsymbol{\beta}, \boldsymbol{\gamma}$ and $\boldsymbol{\delta})$ in Fig. 40:

$$
\begin{aligned}
& \mathbf{P}=\sin \left(\Phi_{1}\right) \sin \left(\Phi_{1}\right) \times \sin \left(\Phi_{2}\right) \sin \left(\Phi_{2}\right) \leftarrow(\text { within eigenstate } \boldsymbol{\alpha}) \\
& +\cos \left(\Phi_{1}\right) \sin \left(\Phi_{1}\right) \times \cos \left(\Phi_{2}\right) \sin \left(\Phi_{2}\right) \leftarrow(\text { within eigenstate } \boldsymbol{\beta}) \\
& +\sin \left(\Phi_{1}\right) \cos \left(\Phi_{1}\right) \times \sin \left(\Phi_{2}\right) \cos \left(\Phi_{2}\right) \leftarrow(\text { within eigenstate } \boldsymbol{\gamma}) \\
& +\cos \left(\Phi_{1}\right) \cos \left(\Phi_{1}\right) \times \cos \left(\Phi_{2}\right) \cos \left(\Phi_{2}\right) \leftarrow(\text { within eigenstate } \boldsymbol{\delta})
\end{aligned}
$$

When we add those four lines together, the result can be factored:

$$
\begin{aligned}
& =\left[\sin \left(\Phi_{1}\right) \sin \left(\Phi_{2}\right)+\cos \left(\Phi_{1}\right) \cos \left(\Phi_{2}\right)\right] \\
& \mathbf{x}\left[\sin \left(\Phi_{1}\right) \sin \left(\Phi_{2}\right)+\cos \left(\Phi_{1}\right) \cos \left(\Phi_{2}\right)\right]
\end{aligned}
$$

There is a trigonometry relationship that allows us to compress those two lines into two functions:

$$
=\cos \left(\Phi_{2}-\Phi_{1}\right) \times \cos \left(\Phi_{1}-\Phi_{1}\right)
$$

$$
=\text { "coincidence rate" }=\cos ^{2}\left(\Phi_{2}-\Phi_{1}\right)
$$

This is how TEW accounts for the Bell test data. The math is astonishingly simple. Our only starting assumption was that a pair of entangled photons follows a Bi-Ray. Our prediction is that the coincidence rate will be $\mathbf{P}=\cos ^{2}\left(\boldsymbol{\Phi}_{2}-\boldsymbol{\Phi}_{1}\right)$. If the 2-photon-Source were changed so it emitted photons orthogonal to one another, then the final coincidence rate would be

$$
P=\sin ^{2}\left(\Phi_{2}-\Phi_{1}\right) \text {. }
$$

TEW shows how the quantum math stretches across space without exceeding the lightspeed limit. TEW obeys the speed-of-light rules but starts earlier than photon emission and therefore appears to go faster than lightspeed. (The assumptions of TEW are different than the assumptions of QM.) By the time a photon pair is emitted, Bi-Rays are long since established. Wave-function collapse (consisting of each 
entangled photon attaching to the same Bi-Ray) occurs as the photons are emitted and not when the photons are measured by Alice and Bob's detectors. But the Bi-Ray is established even before 2-photon emission.

Einstein thought each particle had solid characteristics (hidden variables) even before it was observed. TEW says that is wrong. It is not the intrinsic characteristics of particles, but the elementary waves that constitute the canvas upon which reality is painted. The characteristics of each particle depend on which angle it is observed at. Each person who asks a different question, will get a different answer.

\section{Summary}

This article proposes a new theory of relativity and quantum mechanics, linked by aether.

In part 2 of this article we used the technology of cell towers and computer networks to show that we live within a system of UTC absolute simultaneity that contradicts Einstein's ideas about time. The technology which we experience daily is incompatible with the idea of a space-time continuum, because there is no spatial aspect to time. If Einstein had been correct about time, then all stock markets would crash in less than one second. This led to an affirmation of luminiferous aether as the stationary system in which the absolute simultaneity is rooted. That aether is essential for the remainder of the article, because if Elementary Waves exist, we need to ask what medium they move in. Unlike electromagnetic waves (which move in the opposite direction as Elementary Waves), we need a medium of aether if we are going to propose the existence of zero-energy Elementary Waves travelling everywhere at the speed of light.

In part 3 of this article we built a model to which we gave the name, "Particles follow zero-energy Elementary Waves backwards." As absurd as our model appears at first to be, it is no more absurd than the boxy contraption built by Wilbur and Orville Wright (Fig. 11). Just as the Wright brothers proved their airplane could fly by testing it, similarly, we have demonstrated that our model can "fly" by showing how it works in (i.e. how it explains) five experiments:

4.1 A neutron interferometer experiment.

4.2 A double slit experiment with an explanation of complementarity

4.3 The Schrödinger cat paradox (found above but not in the video)

4.4 The Purcell effect

4.5 A Bell test experiment

Our model has one and only one strength: it can fly! No one else can explain all five experiments!

The boxy contrivance that the Wright brothers built looked so improbable that no one took them seriously until they proved to the world in 1908 that their contraption could fly, turn corners, and remain airborne for an hour, i.e. longer than anyone else's contraption. Their model did not look like an airplane from today. Instead of ailerons they had what they called "warping wings." Instead of an elevator on the tail, they had it sticking out the nose so it looked like a dolphin. Instead of a vertical tail fin, they had two vertical panels in front of the box, and sometimes two more in the back. In order to make the two propellers turn in opposite directions, they used bicycle chains from the engine, and one of the chains crossed over itself like a figure " 8 " to reverse the direction of one propeller vis-à-vis the other propeller powered by a bicycle chain without a figure " 8 ".

Our model probably looks as dissimilar from future models of Elementary Waves, as did the Wright brothers model look dissimilar to today's airplanes. What became clear from their experiments is that an airplane needs to control three orthogonal axes: roll, pitch, and yaw. What becomes clear from our experiments is that TEW needs to control weirdness.

This article is unique in three ways. First, no one has ever used your cell phone to disprove Einstein's ideas about a space-time continuum. Second, no one has ever discovered a mathematical way to abolish 
all quantum weirdness, as we have done. Third, only Lewis E. Little has ever proposed that we look at the other half of nature, the half that consists of zero-energy Elementary Waves. (39-43)

This article asks you a simple question: which is the common-sense approach: the prevailing consensus among physics leaders, or what this article says?

Richard Feynman said that the quantum world behaves differently if we are looking at it than if we close our eyes. We used Feynman's comment as the springboard from which we discovered Elementary Waves. There is an ancient saying, "Seek and you will find." That portrays nature as being interactive and responsive. If nature is not responding, perhaps it means that your eyes are closed.

As we said before, most of the content of this article can be found in two lively and entertaining YouTube videos with no mathematical equations. Those videos are an easier way to learn the subject matter than is this article. One video is 7 or 8 minutes in length, the other is one hour. The equations omitted from the videos are contained in this article. Those videos can be found by searching inside YouTube for the video named "Common-sense rejected by physicists". It is in the YouTube channel named "Elwavetheory" (YouTube might redirect you to "Elwave Theory" in which case you need to insist on "Elwavetheory"). If you can't find the videos, you may have to wait until after they are posted, which should be by October 2021. If you continue to have trouble finding the videos, check the website ElementaryWave.com.

\section{Unidentified Flying Objects (UFOs)}

If we have discovered that Elementary Waves are that part of nature that, at least sometimes, is in control of everything else, can humans figure out some way to control the Elementary Waves? Could a weapon system be built? Could Elementary Waves be recruited in our battle against climate change?

Until recently we did not see any way that humans could control Elementary Waves. But recently in the United States our military aircraft have spotted UFOs (Unidentified Flying Objects). The UFOs have implications for Elementary Waves. The consensus is that there is something out there, but we don't know what or who it is. The United States military reported to Congress in July 2021 that of 141 sightings of UFOs, only one could be explained. They call these objects "unidentified aerial phenomena."

If there are such things flying around, how could it be that they work? Well, it must be that they are using Elementary Waves somehow, because how could you explain something that is so strange? By "strange" we mean that these unidentified aerial phenomena can go from zero to 18 times the speed of sound in a second or two, they can make sharp turns, then abruptly hover. It is not within the capability of any normal technology that we know of to move so fast and effortlessly. 


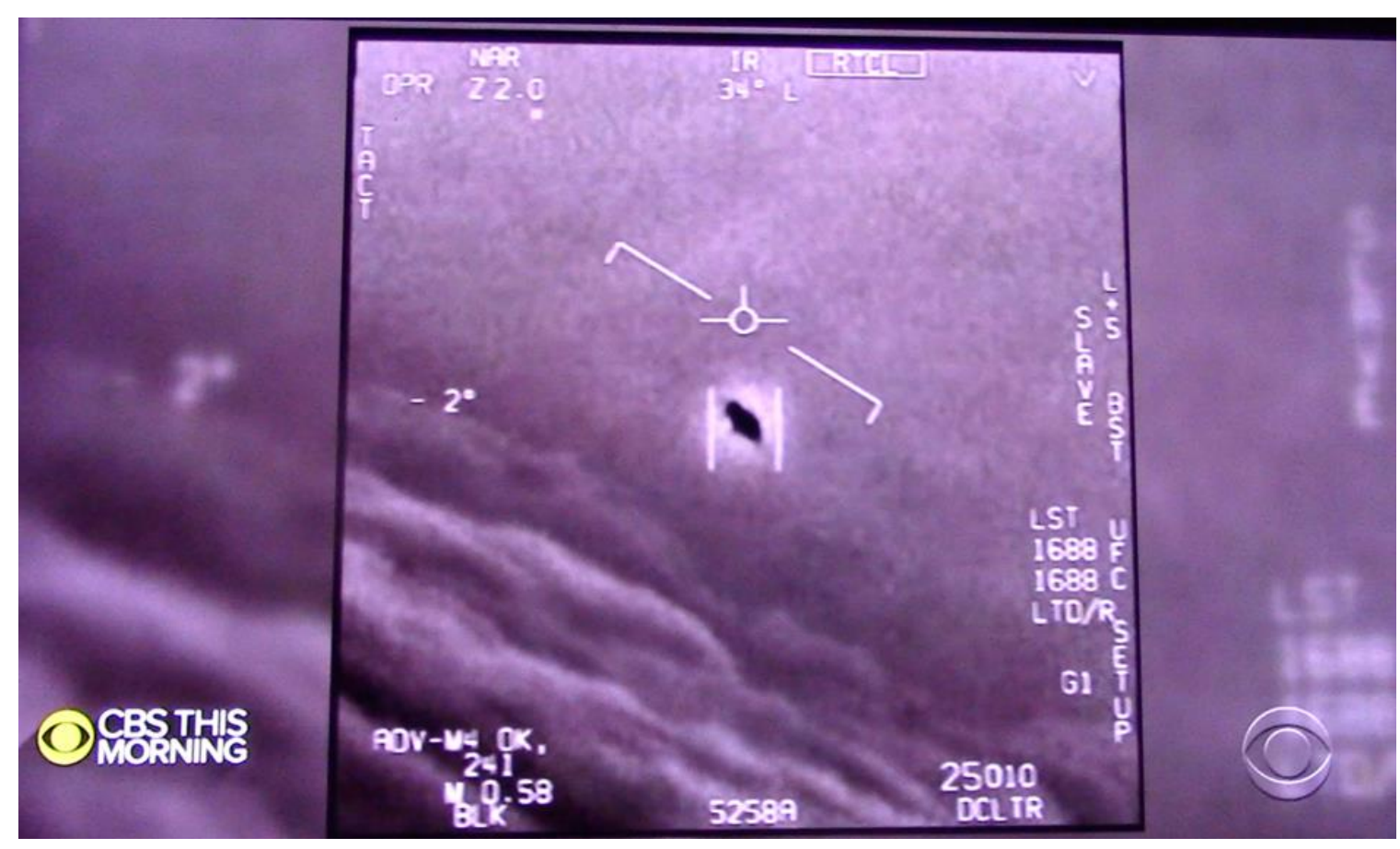

Fig. 41. Unidentified Flying Object (UFO) seen from American fighter jet and reported to U.S. Congress.

If it were not for these UFOs, we would not believe that it is possible for humans to gain control over Elementary Waves, and thereby control the system that may control everything else. We can imagine now that we might be able to study UFOs and learn from them how to gain control over Elementary Waves. So, because of the UFOs we need to be open to the possibility that someone has learned how to control Elementary Waves and therefore perhaps we could learn that and recruit the Elementary Waves in our battle against climate change: i.e., our task of drawing down carbon out of the atmosphere, as Paul Hawken proposes in his book Drawdown.

\section{Acknowledgement}

The author thanks Franco Selleri, who invented absolute simultaneity, and Lewis E. Little, who invented the idea of TEW.

\section{Conflict of interest}

The author declares that there is no conflict of interest regarding the publication of this article.

\section{Author Biography}

The author lives in Connecticut, USA. He has graduated with diplomas from Harvard, Yale, Brown, and Case Western Reserve Universities and has been trained in mathematics, and several sciences. He has worked and taught in many settings, including as an epidemiologist on the faculty of the National Institutes of Health in Bethesda, Maryland, USA. Most recently worked as an M.D. physician. In 2020 he retired and has devoted his time to TEW and welding steel sculpture. 


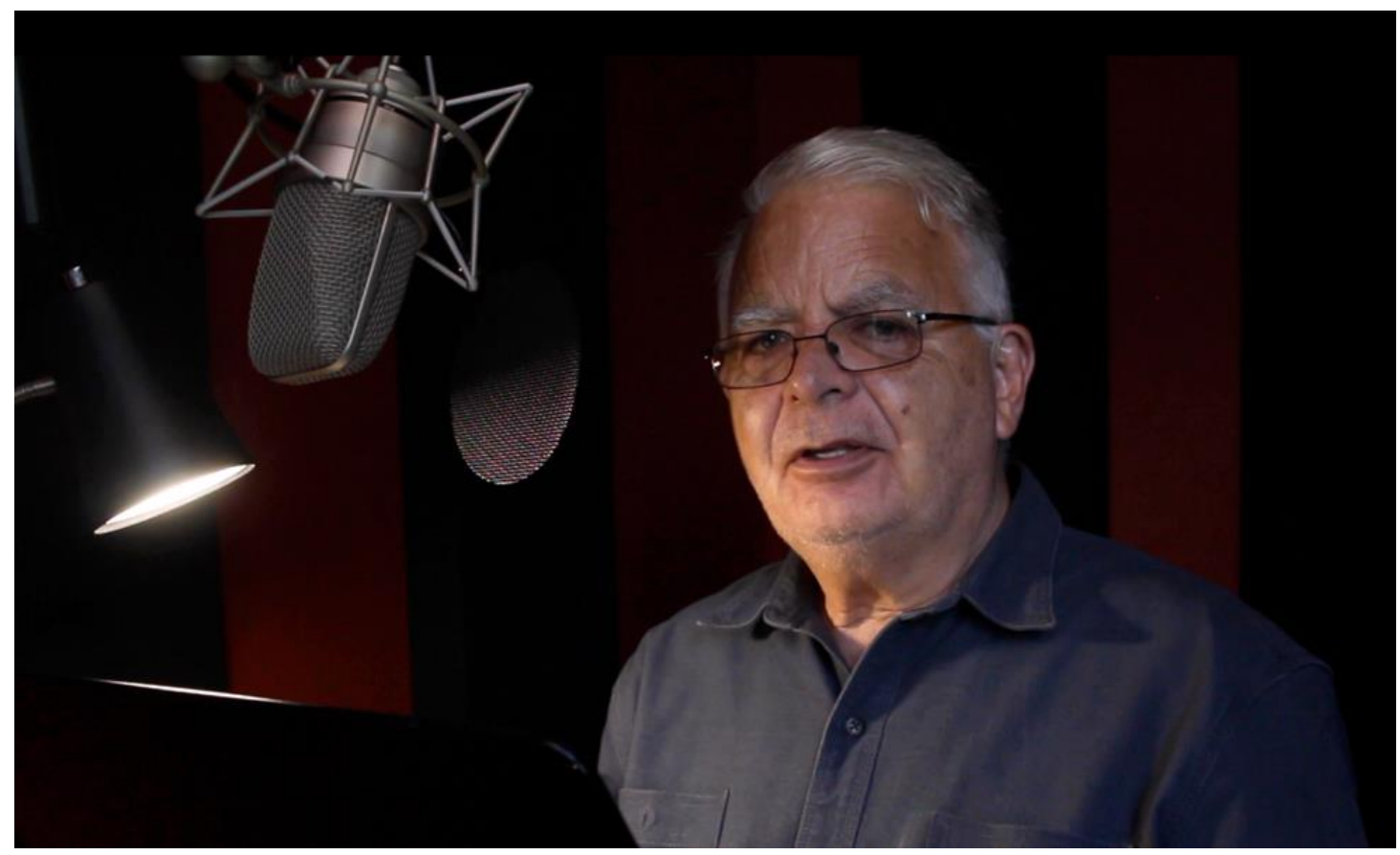

Jeffrey H. Boyd in 2021, recording the YouTube videos that accompany this article.

\section{References}

1. J. Baggott, The Quantum Story: a history in 40 moments, Oxford University Press, 2011. ISBN:978-0-19-956684-6

2. A. Becker, What Is Real? Basic Books, 2018. ISBN:978-0-19-956684-6

3. M. Born, "On the quantum mechanics of collisions," in J. A. Wheeler and W. H. Zurek (eds.), Quantum Theory and Measurement, Princeton, pp.50-55, 1983. ISBN 978-0-691-08316-2.

4. J. H. Boyd, "PDE boundary conditions that eliminate quantum weirdness: a mathematical game inspired by Kurt Gödel and Alan Turing," Journal of Advances in Mathematics, vol. 20, pp. 211-213, 2021. DOI: 10.24297/jam.v20i.9042

5. J. H. Boyd, "PDE boundary conditions that eliminate quantum weirdness," a YouTube video, 2021, at https://www.youtube.com/watch?v=czinM1gSgKE

6. J. H. Boyd, "Six reasons to discard wave particle duality." Journal of Advances in Chemistry, 18, 1-29, 2021. DOI: 10.24297/jac.v18i.8948

7. J. H. Boyd, "6 reasons to discard wave particle duality," a YouTube video, 2021. https://www.youtube.com/watch?v=LD4xXI5gMzg\&t=2s

8. J. H. Boyd, "The Periodic Table needs negative orbitals in order to eliminate quantum weirdness," Journal of Advances in Chemistry, vol. 17, pp.88-125, 2020. DOI: 10.24297/jac.v17i.8865

9. J. H. Boyd, "There are two solutions to the equations of Feynman's Quantum Electrodynamics (QED); the newly discovered solution is free of quantum weirdness," Journal of Advances in Physics, vol. 18, pp. 39-57, 2020. DOI: 10.24297/jap.v18i.8831

10. J. H. Boyd, "If the propagator of QED were reversed, the mathematics of Nature would be much simpler," Journal of Advances in Mathematics, vol. 18, pp. 129-153, 2020. DOI: 10.24297/jam.v18i.8746 
11. J. H. Boyd, "A tiny, counterintuitive change to the mathematics of the Schrödinger wavepacket and Quantum ElectroDynamics could vastly simplify how we view Nature," Journal of Advances in Physics, vol. 17, pp. 169-203, 2020. DOI: 10.24297/jap.v17i.8696

12. J. H. Boyd, "New Schrödinger wave mathematics changes experiments from saying there is, to denying there is quantum weirdness," Journal of Advances in Mathematics, vol. 18, pp. 82117, 2020. DOI: 10.24297/jam.v18i.8656

13. J. H. Boyd, "Decrypting the central mystery of quantum mathematics: Part 1. The double slit experiment," Journal of Advances in Mathematics, 2 vol. 17, pp. 255-282, 2019. DOI: 10.24297/jam.v17i0.8475

14. J. H. Boyd, "Decrypting the Central Mystery of Quantum Mathematics: Part 2. A mountain of empirical data supports TEW," Journal of Advances in Mathematics, vol. 17, pp. 283-314, 2019. DOI: $10.24297 /$ jam.v17i0.8489

15. J. H. Boyd, "Decrypting the central mystery of quantum mathematics: Part 3. A non-Einstein, non-QM view of Bell test experiments," Journal of Advances in Mathematics, vol. 17, pp. 315331, 2019. DOI: 10.24297/jam.v17i0.8490

16. J. H. Boyd, "Decrypting the central mystery of quantum mathematics: Part 4. In what medium do Elementary Waves travel?" Journal of Advances in Mathematics, vol. 17, pp. 332-351, 2019. DOI: $10.24297 / j a m . v 17 i 0.8491$

17. J. H. Boyd, "The quantum world is astonishingly similar to our world," Journal of Advances in Physics, vol. 14, pp. 5598-5610, 2018. DOI: 10.24297/jap.v14i2.7555

18. J. H. Boyd, "The von Neumann and double slit paradoxes lead to a new Schrödinger wave mathematics," Journal of Advances in Physics, vol.14, pp. 5812-5834, 2018. DOI: 10.24297/jap.v14i3.7820

19. J. H. Boyd, "The Boyd Conjecture," Journal of Advances in Physics, vol. 13, pp. 4830-4837, 2017. DOI: 10.24297/jap.v13i4.6038

20. J. H. Boyd, "A symmetry hidden at the center of quantum mathematics causes a disconnect between quantum math and quantum mechanics," Journal of Advances in Mathematics, vol. 13, pp. 7379-7386, 2017. DOI: 10.24297/jam.v13i4.6413

21. J. H. Boyd, "Paul Dirac's view of the Theory of Elementary Waves," Journal of Advances in Physics, vol. 13, pp. 4731-4734, 2017. DOI: 10.24297/jap.v13i3.5921

22. J. H. Boyd, "A paradigm shift in mathematical physics, Part 1: The Theory of Elementary Waves (TEW)," Journal of Advances in Mathematics, vol. 10, pp. 3828-3839, 2015. DOI: 10.24297/jam.v10i9.1908

23. J. H. Boyd, "A paradigm shift in mathematical physics, Part 2: A new local realism explains Bell test \& other experiments," Journal of Advances in Mathematics, vol. 10, pp. 3828-3839, 2015. DOI: 10.24297/jam.v10i9.1884

24. J. H. Boyd, "A paradigm shift in mathematical physics, Part 3: A mirror image of Feynman's quantum electrodynamics (QED)," Journal of Advances in Mathematics, vol. 11, pp. 3977-3991, 2015. DOI: $10.24297 /$ jam.v11i2.1283

25. J. H. Boyd, "A paradigm shift in mathematical physics, Part 4: Quantum computers and the local realism of all 4 Bell states," Journal of Advances in Mathematics, vol. 11, pp. 5476-5493, 2015. DOI: 10.24297/jam.v11i7.1224

26. J. H. Boyd, "The Theory of Elementary Waves eliminates Wave Particle Duality," Journal of Advances in Physics, vol. 7, pp. 1916-1922, 2015. DOI: 10.24297/jap.v7i3.1576 
27. J. H. Boyd, "A new variety of local realism explains a Bell test experiment," Journal of Advances in Physics, vol. 8, pp. 2051-2058, 2015. DOI: 10.24297/jap.v8i1.1541

28. J. H. Boyd, "A proposed physical analog of a quantum amplitude," Journal of Advances in Physics, vol. 10, pp. 2774-2783, 2015. DOI: 10.24297/jap.v10i3.1324

29. J. H. Boyd, "Re-thinking a delayed choice quantum eraser experiment: a simple baseball model," Physics Essays, vol. 26, pp. 100-109, 2013. DOI: 10.4006/0836-1398-26.1.100

30. J. H. Boyd, "Re-thinking Alain Aspect's 1982 Bell test experiment with delayed choice," Physics Essays, vol. 26, pp. 582-591, 2013. DOI: 10.4006/0836-1398-26.1.100 10.4006/0836-139826.4.582

31. J. H. Boyd, "Rethinking a Wheeler delayed choice gedanken experiment," Physics Essays, vol. 25, pp. 390-396, 2012. DOI: 10.4006/0836-1398-25.3.390

32. A. Einstein, "Einstein 1920 - Does aether exist?" ProTruth channel, Dec 32014. https://www.youtube.com/watch?v=yCm6eLP9zRw

33. H. Minkowski and the postulate of relativity, Arch. Hist. Exact Sci. vol. 51, pp. 273-314 (1997). DOI: 10.1007/BF00518231

34. L. de Broglie, Research on the Theory of Quanta, translated by André Michaud and Fritz Lewertoff, Montreal: Minkowski Institute Press, 2021. ISBN: 1-927763-99-5.

35. Olival Freire. Interview with Dr. F. Selleri. American Institute of Physics: Oral History Interviews. https://www.aip.org/history-programs/niels-bohr-library/oral-histories/28003-1. and https://www.aip.org/history- programs/niels-bohr-library/oral-histories/28003-2. (accessed July 15, 2019)

36. R. G. Hulet, E. S. Hilfer, and D. Kleppner, "Inhibited spontaneous emission by a Rydberg atom," Physical Review Letters, vol. 55, pp. 2137-2140, 1985. https://doi.org/10.1103/PhysRevLett.55.2137

37. H. Kaiser, R. Clothier, S. Werner, et. al., "Coherence and spectral filtering in neutron interferometry," Physical Review A, vol. 45, pp. 31-42, 1992. DOI: 10.1103/PhysRevA.45.31

38. T. S. Kuhn, The Structure of Scientific Revolutions, (Chicago: U. of Chicago Press, 1970. ISBN 978-0-226-45803-8.

39. L. E. Little, The Theory of Elementary Waves, (New Classics Library, Gainsville, GA), 2009. ISBN: 978-0-932750-84-6.

40. L. E. Little, "Theory of Elementary Waves," Physics Essays, vol. 9, pp. 100-134, 1996. DOI: 10.4006/1.3029212

41. L. E. Little, "Theory of Elementary Waves @ JPL, Feb 2000," https://www.youtube.com/watch?v=3_9LBORzgWg

42. L. E. Little "Introduction to Elementary Waves," 2016. https://www.youtube.com/watch?v=xx5V03iCbAo\&t=16s

43. L. E. Little, "We have seen these waves," 2016. https://www.youtube.com/watch?v=xWMiNsD_xdM\&t=5s

44. $\quad \mathrm{H}$. A. Lorentz. Electromagnetic phenomena in a system moving with any velocity smaller than that of light. Proceedings of the Royal Netherlands Academy of Arts and Sciences, 6, 809-831, 1904.

45. A. A. Michelson and Edward W. Morley. Relative motion of the earth and the luminiferous aether. American Journal of Science, 34, 335-345, 1887. DOI: 10.2475/ajs.s3-34.203.333 
46. N. D. Mermin, "Is the moon there when nobody looks? Reality and the quantum theory," Physics Today, 38, 38-47 (1985). DOI: 10.1063/1.880968

47. H. Minkowski. Space and time, in Hendrik A.Lorentz, Albert Einstein, Hermann Minkowski and H. Weyl, The Principle of Relativity (Dover Publications, 1952) pp. 73-81. ISBN-13 798-0-48660081-9.

48. Petit, G., Wolf, P., "Relativistic theory for syntonization of clocks in the vicinity of the earth," Proceedings of the 26th Annual Precise Time and Time Interval Systems and Applications Meeting, Reston, Virginia, December 1994, pp. 381-392. https://www.ion.org/publications/abstract.cfm?articlelD=14316

49. J. R. Pierce, Almost All About Waves, New York: Dover Books, 1974. ISBN: 0-486-45302-2.

50. E. M. Purcell, "Proceedings of the American Physical Society: Spontaneous Emission Probabilities at Ratio Frequencies" Physical Review. American Physical Society 69 (11-12): 681. http://pages.erau.edu/ reynodb2/colloquia/Purcell_1946_SpontaneousEmission.pdf

51. M. G. Sagnac. On the proof of the reality of the luminiferous aether by the experiment with a rotating interferometer (in French) Comptes Rendus, 157, 1410-1413, 1913.

52. M. G. Sagnac. The demonstration of the luminiferous aether by an interferometer in uniform rotation (in French). Comptes Rendus, 157, 708-710, 1913.

53. M. G. Sagnac. On by the Rotating Interferometer Experiment (in French). Journal de Physics, 4, 177, 1914.

54. E. Schrödinger, Collected Papers on Wave Mechanics, Montreal: Minkowski Institute Press, 2020, ISBN: 978-1-927763-81-0.

55. E. Schrödinger, Abhandlunen zur Wellenmechanik, published by Leipzig: Johann Ambrose Barth, 1928.

56. F. Selleri. Lezioni di Relativita (Bari, Italy: Progedit s.n.c., 2003) ISBN 88-88550-33-X

57. F. Selleri. Lessons in Relativity, chapters 3 to 5, translated by Dominick Scaramuzzino, Nov-Dec 2016 and Jan 2017, Bethany, CT, USA.

58. F. Selleri. The Sagnac effect, once more. Proceedings of the NPA, 9, 525-527, 2012.

59. F. Selleri. Eight proofs of absolute simultaneity. Proceedings of the NPA, 6, 1-10, 2010

60. F. Selleri. Recovering the Lorentz aether. Apeiron, 11, 246-281, 2004.

61. F. Selleri. Our absolute velocity, In Physical Interpret. of Relativity Theory, (C. Duffy editor), British Soc. Philosophy of Science, London 2002.

62. F. Selleri. On the direct observability of quantum waves. Foundations of Physics 12, 11, 1982. DOI: 10.1007/BF01300548

63. F. Selleri. Noninvariant one-way velocity of light. Foundations of Physics, 26 641-664, 1996. DOI: 10.1007/BF02058237

64. J. von Neumann, Mathematical Foundations of Quantum Mechanics, translated by Robert T. Beyer, (Princeton NJ: Princeton University Press, c1955). ISBN: 0-691-02893-1

65. A. Wegener, The Origin of Continents and Oceans, translated by John Biram, New York, Dover Publications Inc.1966, Library of Congress Catalog Card Number: 66-28270.

66. S. A. Werner, R. Clothier, H. Kaiser, et.al., "Spectral filtering in neutron interferometry," Physical Review Letters, vol. 67, pp.683-686, 1991. DOI: 10.1103/PhysRevLett.67.683 